\title{
Efficient Catalytic Effects of Lewis Acids in the 1,3-Dipolar Cycloaddition Reactions of Carbonyl Ylides with Imines
}

\author{
Hiroyuki Suga* Yasutaka Ebiura, Kazuaki Fukushima, Akikazu Kakehi, and Toshihide Baba
}

Department of Chemistry and Material Engineering, Faculty of Engineering, Shinshu University, Wakasato, Nagano 380-8553, Japan, Wakayama Medical University, 811-1 Kimiidera, Wakayama 641-8509, Japan, and Department of Environmental Chemistry \& Engineering, Interdisciplinary Graduate School of Science \& Engineering, Tokyo Institute of Technology, G1-14, 4259 Nagatsuta, Midori-ku, Yokohama 226-8503, Japan sugahio@shinshu-u.ac.jp

\section{Supporting Information}

Table of Contents

Experimental Section (General Methods, Materials, and Computational Methods).... S2

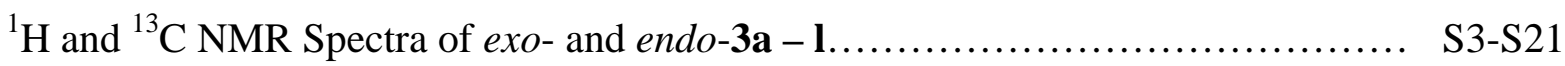

${ }^{1} \mathrm{H}$ and ${ }^{13} \mathrm{C}$ NMR Spectra of exo- and endo-6c............................. S22-S23

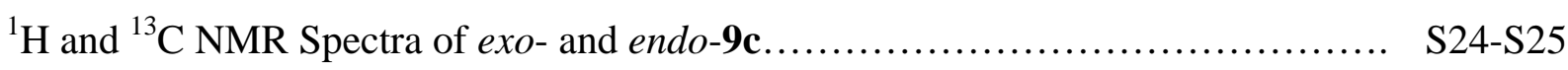

${ }^{1} \mathrm{H}$ and ${ }^{13} \mathrm{C}$ NMR Spectra of exo- and endo-11c............................. S26-S27

X-ray crystallographci analysis of exo-3g............................... S28-S49

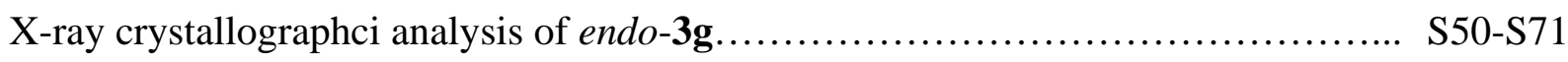

Calculations with RHF/3-21G method................................ S72-S74

Calculations with ONIOM(B3LYP/6-31G(d):PM3) method.................... S74-S88

Proposed mechanisms for formation of compounds 4 and $7 \ldots \ldots \ldots \ldots \ldots \ldots \ldots \ldots \ldots . . . \ldots \ldots$

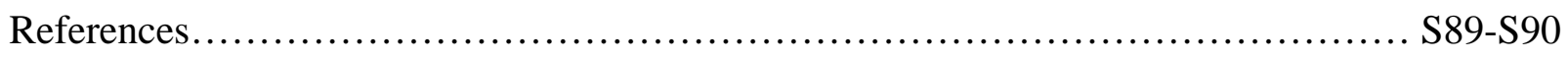


Experimental Section (General Methods, Materials, and Computational Methods)

General Methods. Melting points are uncorrected. IR spectra were taken with FT/IR spectrophotometer. ${ }^{1} \mathrm{H}$ NMR spectra were run at $400 \mathrm{MHz}$. Chemical shifts are expressed in parts per million downfield from tetramethylsilane as an internal standard. ${ }^{13} \mathrm{C}$ NMR spectra were recorded at $100 \mathrm{MHz}$ using broadband proton decoupling. Chemical shifts are expressed in parts per million downfield from tetramethylsilane, using the middle resonance of $\mathrm{CDCl}_{3}(77.0 \mathrm{ppm})$ as an internal standard. For preparative column chromatography, Wakogel C-300HG was employed. Medium-pressure liquid chromatography was carried out using a column packed with Wakogel C-300HG. All reactions were carried out under an argon atmosphere in dried glassware.

Materials. $\quad o$-(Methoxycarbonyl)- $\alpha$-diazoacetophenone (1) and diazomethyl 2,3,4,5-tetrachloro-6-methoxycarbonylphenly ketone (10) were prepared by the procedure in the previous paper. ${ }^{1}$ 1-Diazo-5-phenyl-2,5-pentanedione (5) and 1-diazo-2,5-hexanedione (8) were prepared according to the procedure reported by Padwa. ${ }^{2}$ Imines except $2 \mathbf{l}$ were prepared according to the procedure in the literature. ${ }^{3}$ Imine $\mathbf{2 l}$ was prepared by the procedure reported by Nguyen. ${ }^{4} \quad \mathrm{Rh}_{2}(\mathrm{OAc})_{4}$ and Lewis acids except rare earth metal triflates were commercially available, and used without further purification. Rare earth metal triflates utilized for the reaction were individually dried in a schlenk tube at $200{ }^{\circ} \mathrm{C}$ in vacuo for $12 \mathrm{~h}$ before use. Powdered $4 \AA$ molecular sieves was commercially available and dried in vacuo at $250{ }^{\circ} \mathrm{C}$ for $12 \mathrm{~h}$ before use. $\mathrm{CH}_{2} \mathrm{Cl}_{2}$ was purified by distillation first from $\mathrm{CaCl}_{2}$ and then $\mathrm{CaH}_{2}$ under argon. Toluene and diethyl ether were freshly distilled from a sodium benzophenone still under argon. $t$-Butyl methyl ether (anhydrous) was commercially available, and used without further purification.

\section{Computational Methods}

All the calculations were performed using Gaussian $03 \mathrm{~W}$ program. ${ }^{5}$ Calculations of energies and coefficients of molecular orbitals for carbonyl ylide $\mathbf{A}$, substituted imines $\mathbf{2 c}, \mathbf{2 d}$, and $\mathbf{2 e}$, and the complexes of them with $\mathrm{ZnCl}_{2}$ were carried out at the $\mathrm{RHF} / 3-21 \mathrm{G}$ level of theory. Accurate geometries and energies for uncatalyzed and $\mathrm{ZnCl}_{2}$-catalyzed reactions were calculated by two-layered ONIOM method. 6 Geometries of carbonyl ylide $\mathbf{A}$, imine $\mathbf{2 c}, \mathrm{ZnCl}_{2}$, a complex of $\mathbf{2 c}$ with $\mathrm{ZnCl}_{2}\left(2 \mathrm{c}-\mathrm{ZnCl}_{2}\right)$, a complex of $\mathbf{A}$ with $\mathbf{2 c}$, a complex of A with $\mathbf{2} \mathbf{c}-\mathrm{ZnCl}_{2}$, transition states of the reactions, a complex of exo-3c with $\mathrm{ZnCl}_{2}$, and exo-3c were optimized with ONIOM(B3LYP/6-31G(d):PM3) method. A carbonyl ylide moiety, a carbonyl group, and a methoxy group in $\mathbf{A}$, a imine group in $\mathbf{2 c}, \mathrm{ZnCl}_{2}$, and the corresponding parts in the complexes, the transition states, and exo-3c were calculated as high layers. The residual benzene ring moieties were calculated as low layers. Link atoms of these layers were specified as $\mathrm{H}$ atoms on the calculation of model systems. All the stationary points were characterized as minima or transition states by frequency calculations. Calculations of thermodynamic parameters were carried out under $298.15 \mathrm{~K}$ and $1 \mathrm{~atm}$. Correction of vibrational frequency with scaling factors was not carried out. 
${ }^{1} \mathrm{H}$ NMR of Compound exo-3a

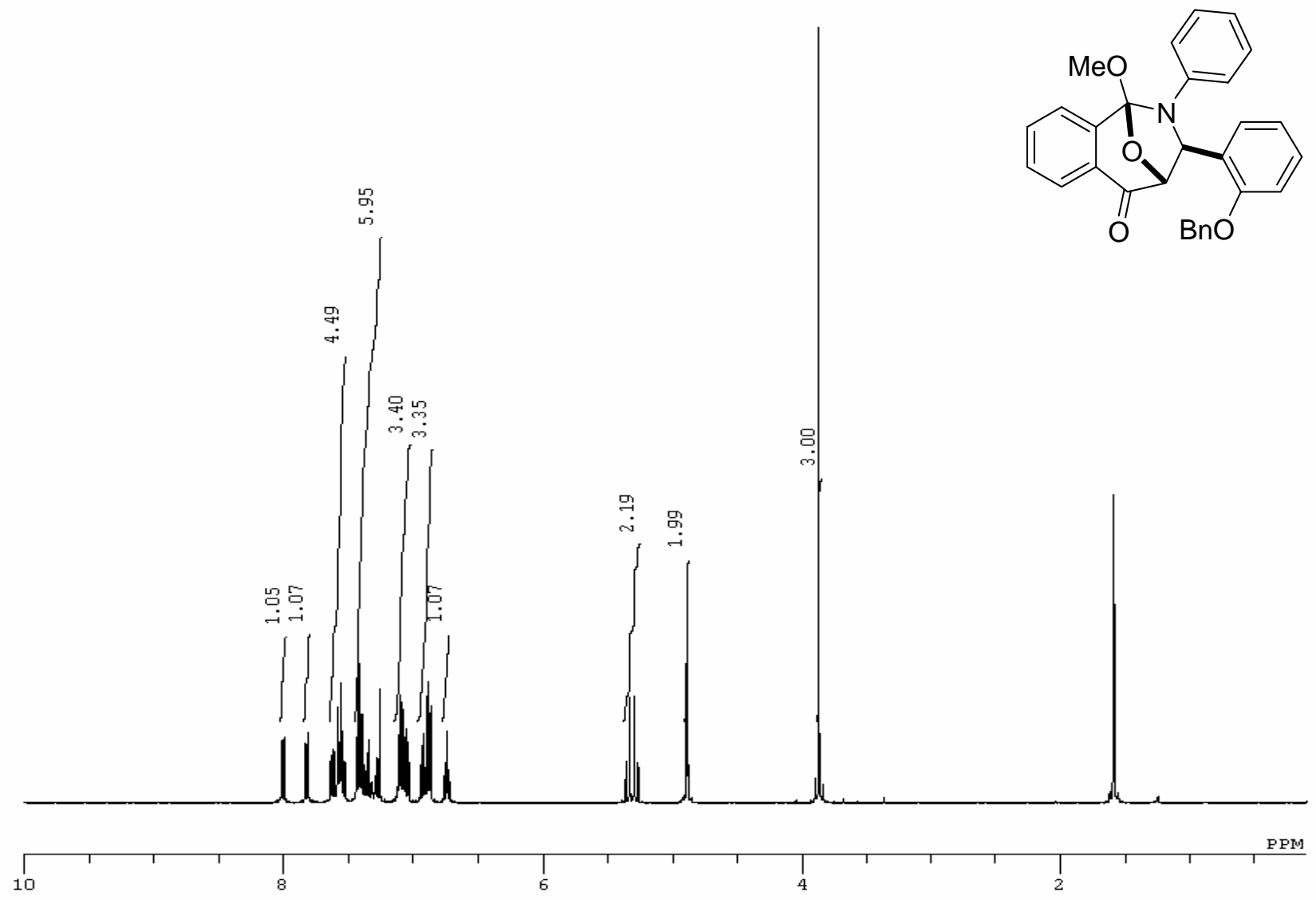

${ }^{13} \mathrm{C}$ NMR of Compound exo-3a

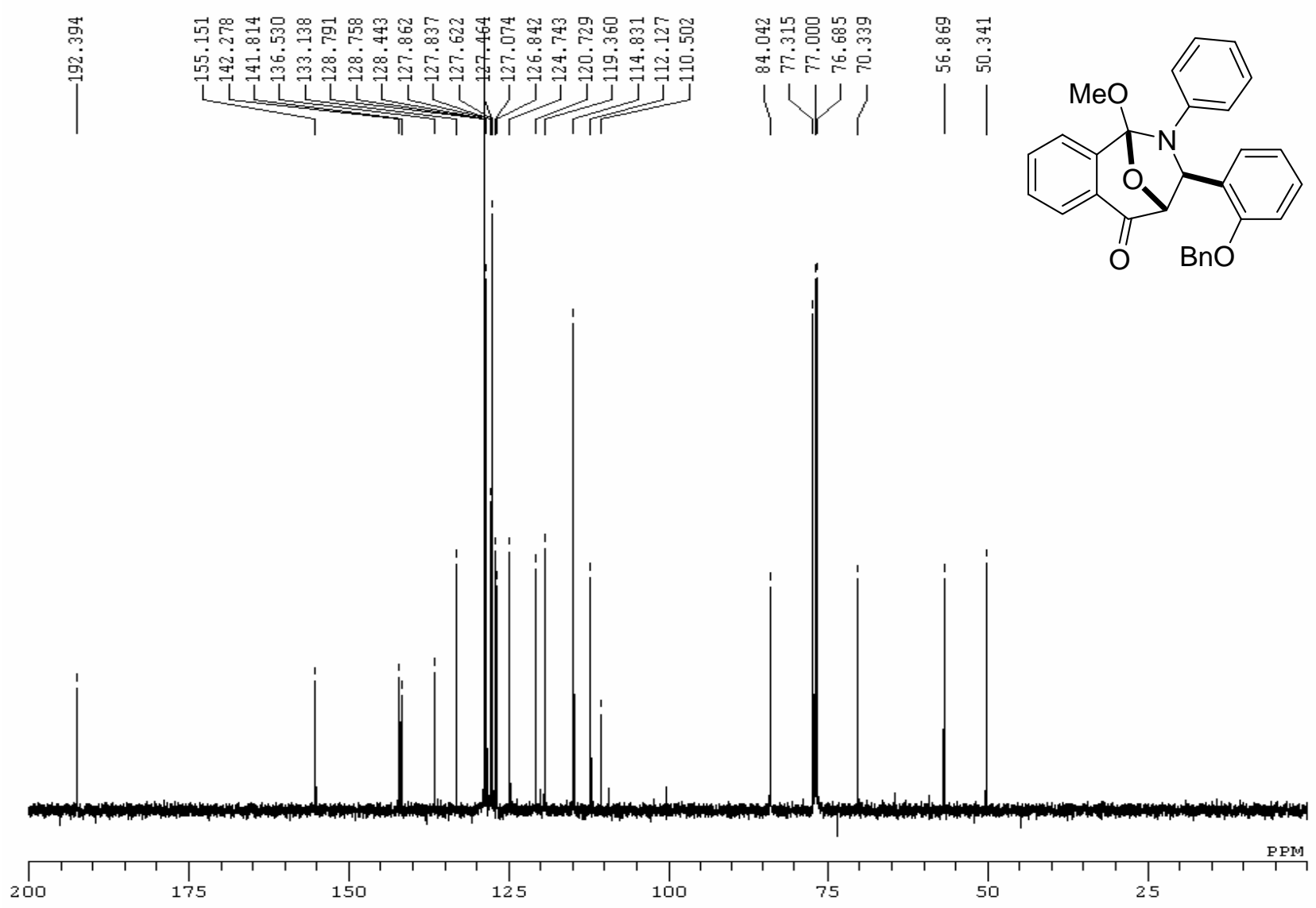


${ }^{1} \mathrm{H}$ NMR of Compound endo-3a

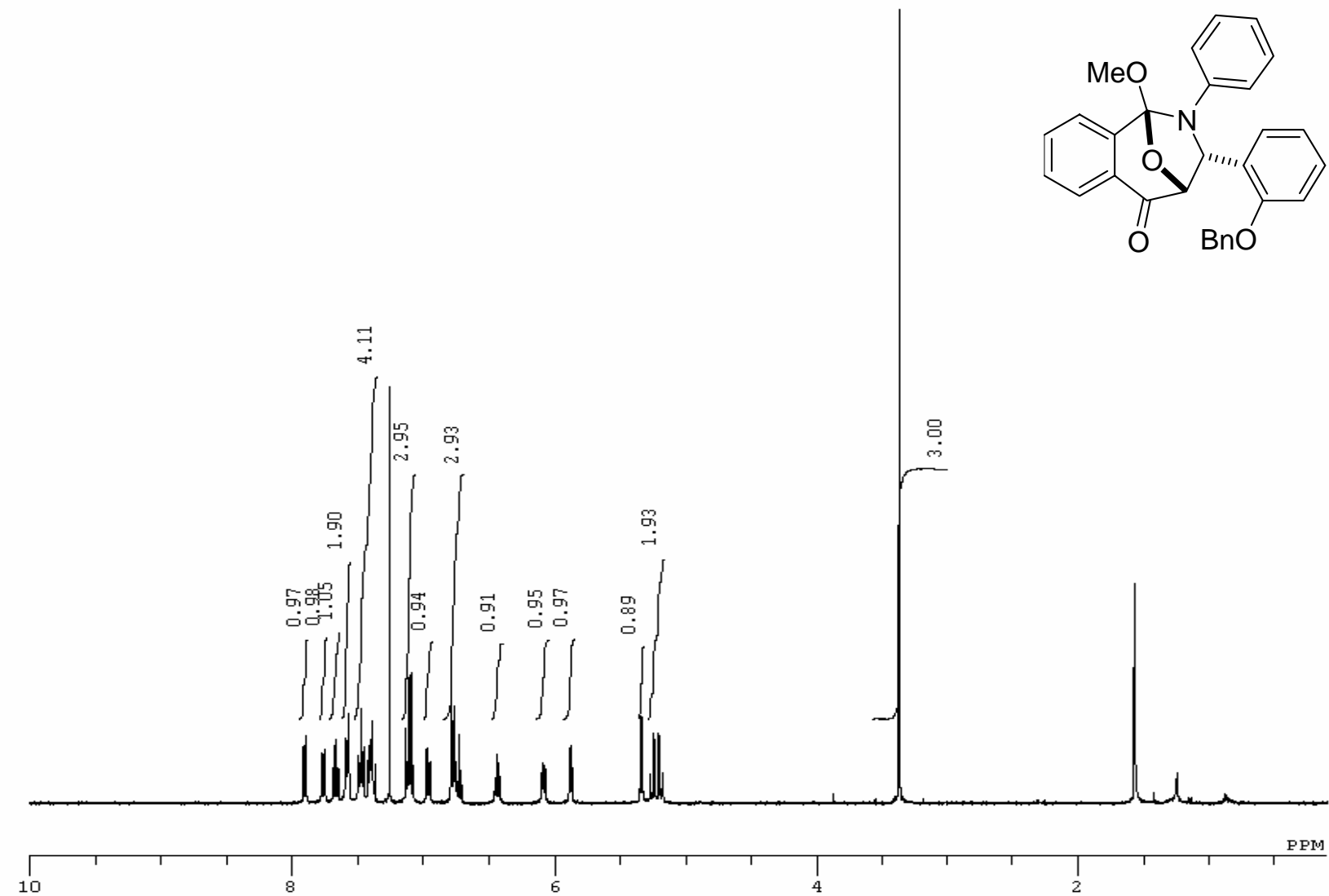

${ }^{13} \mathrm{C}$ NMR of Compound endo-3a

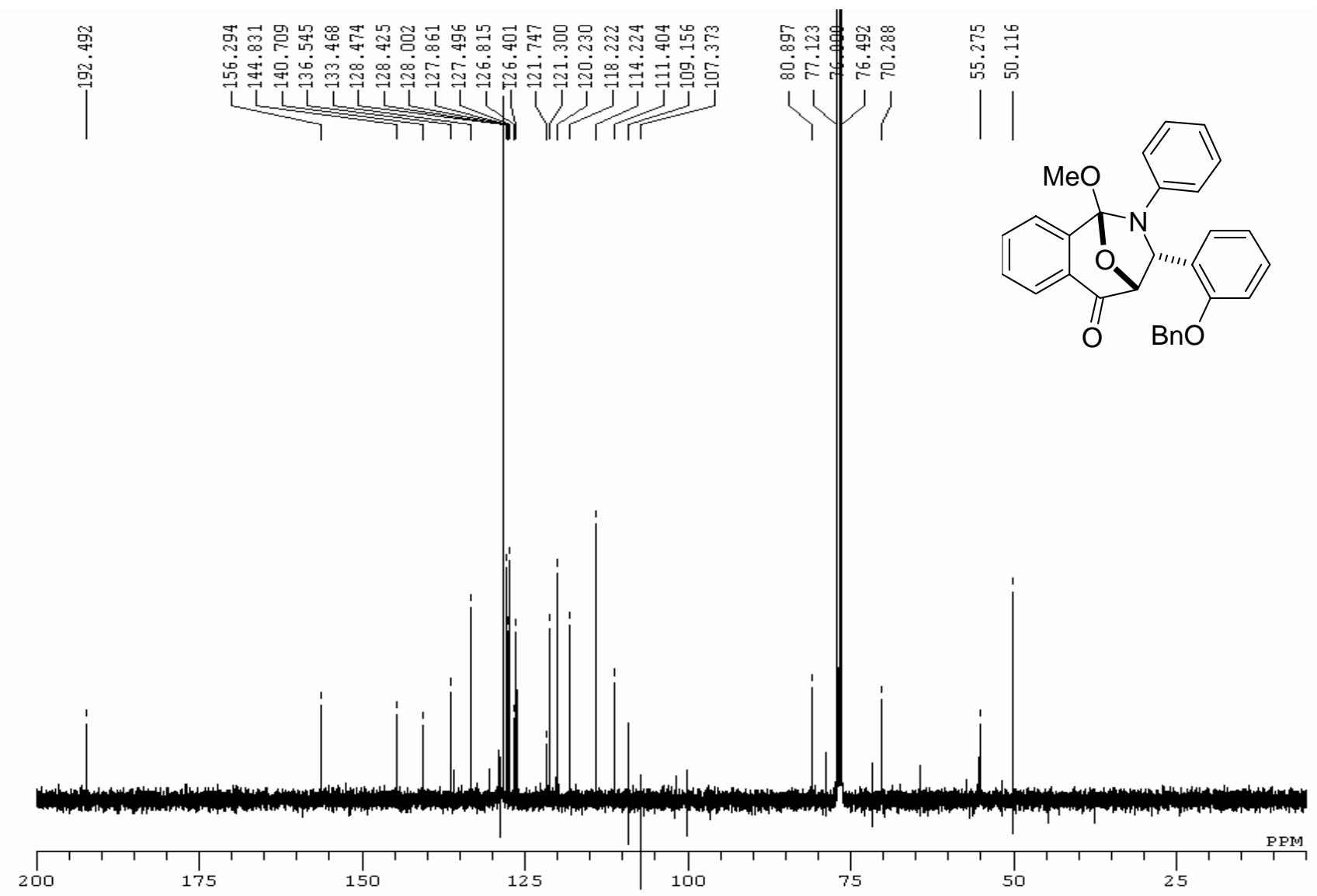


${ }^{1} \mathrm{H}$ NMR of Compound exo-3b

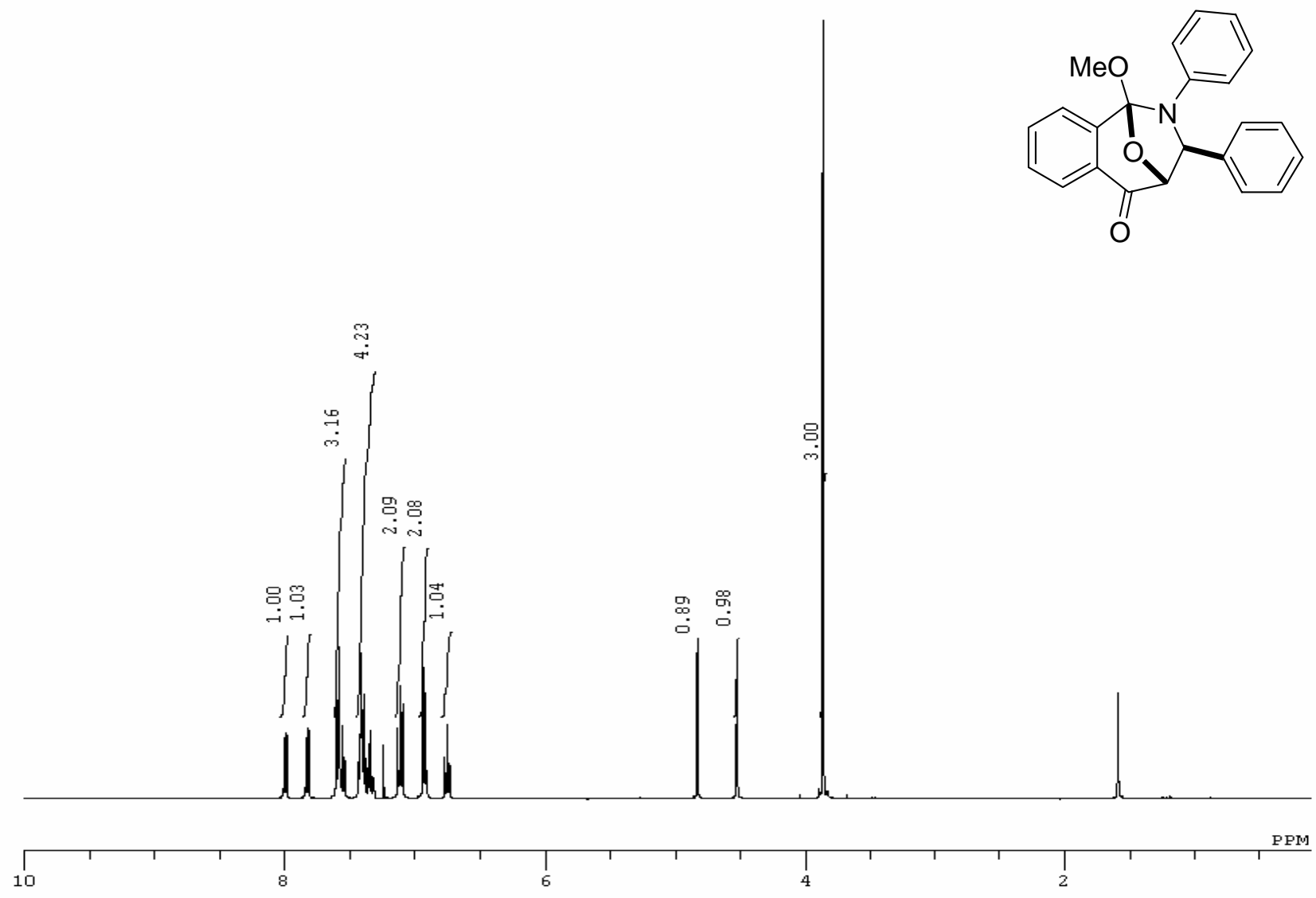

${ }^{13} \mathrm{C}$ NMR of Compound exo-3b

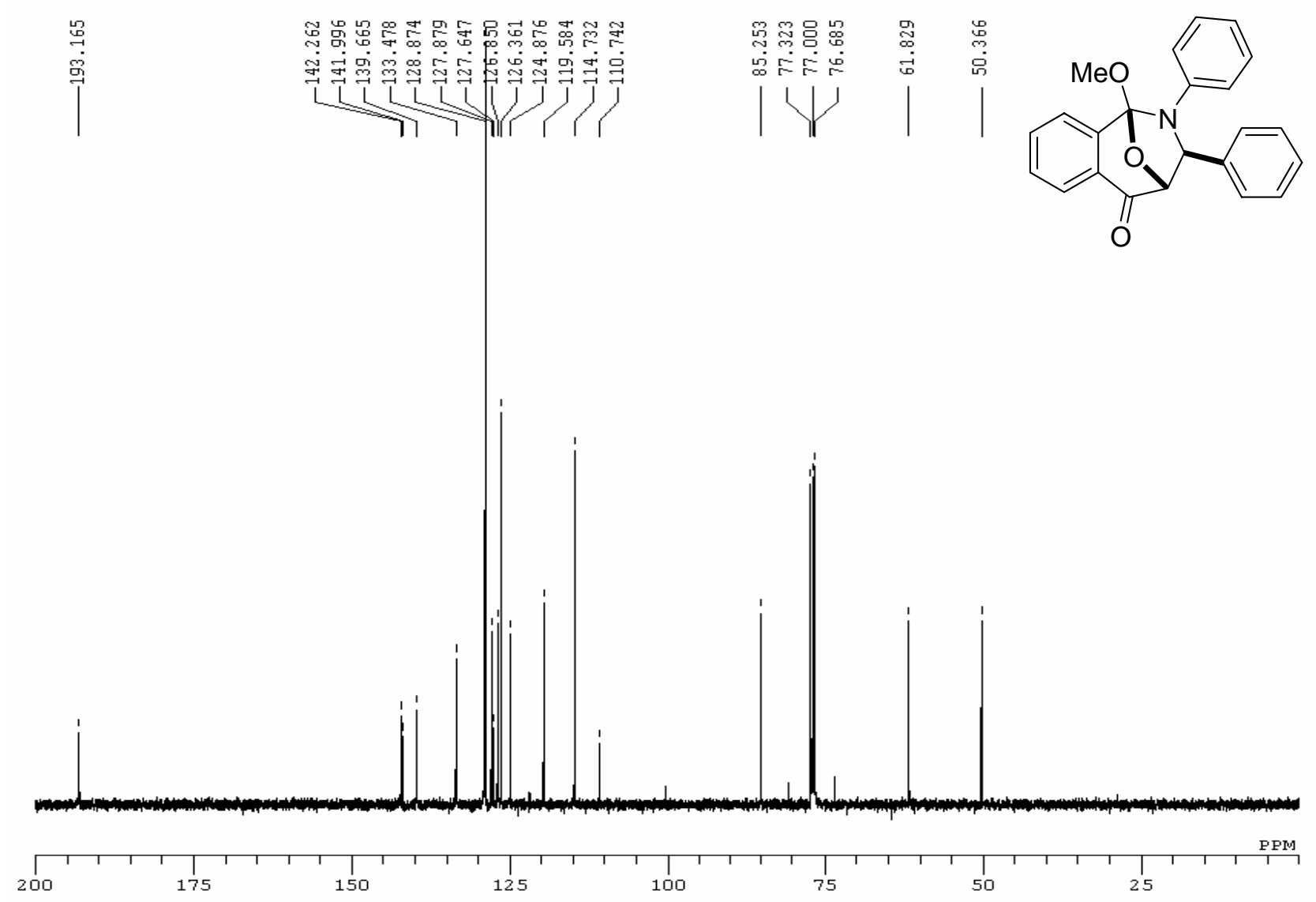


${ }^{1} \mathrm{H}$ NMR of Compound endo-3b

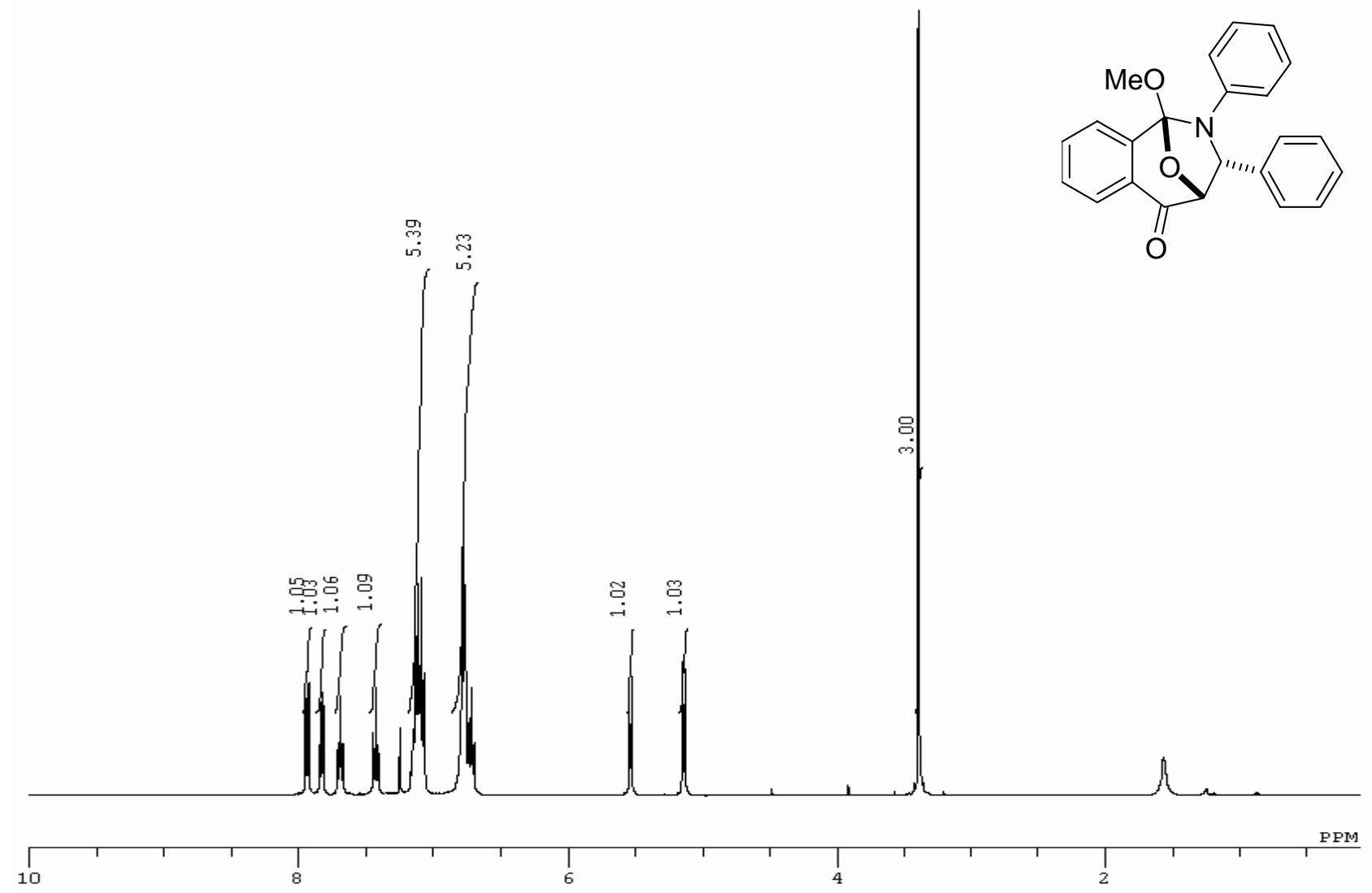

${ }^{13} \mathrm{C}$ NMR of Compound endo-3b

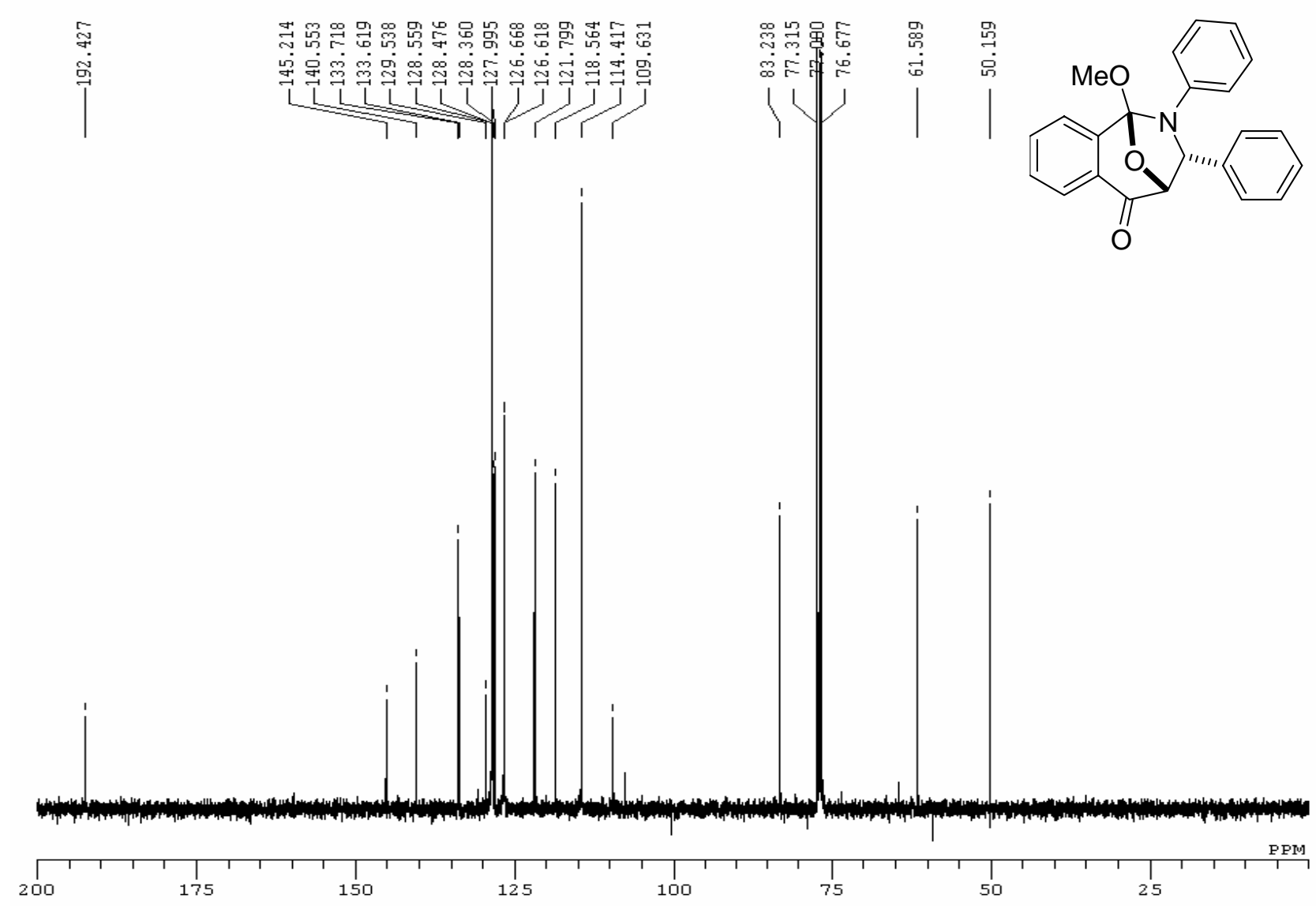


${ }^{1} \mathrm{H}$ NMR of Compound exo-3c

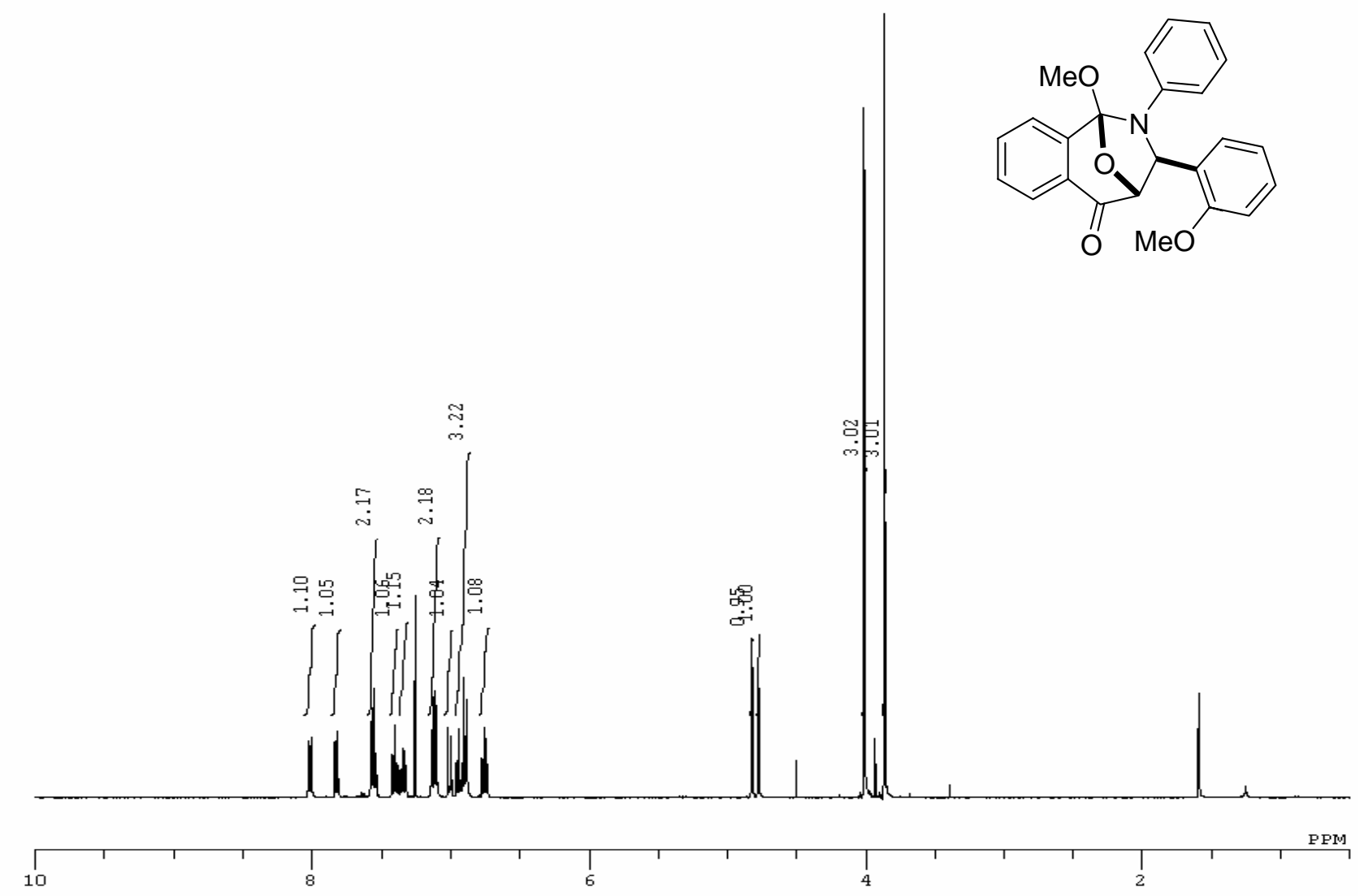

${ }^{13} \mathrm{C}$ NMR of Compound exo-3c

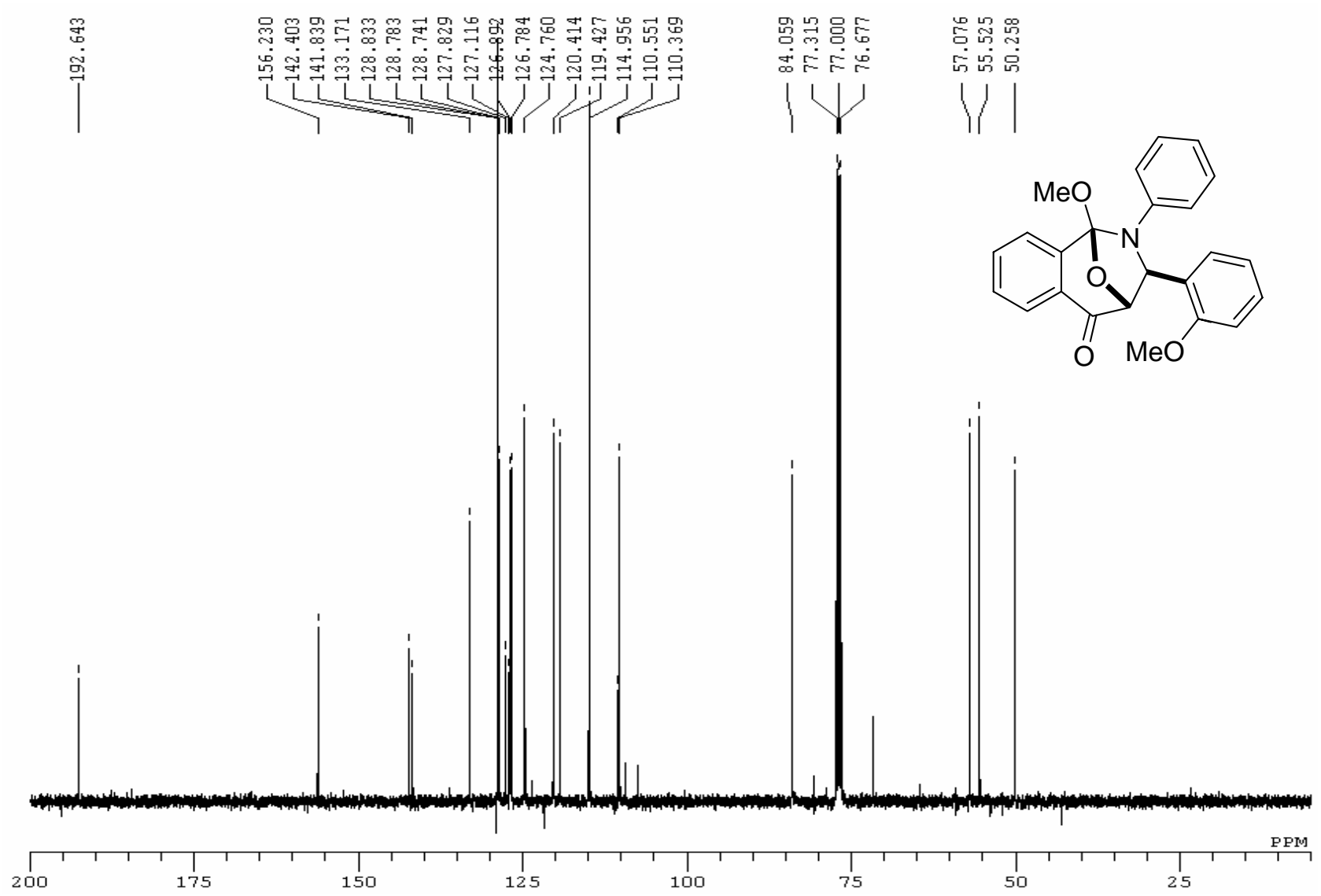


${ }^{1} \mathrm{H}$ NMR of Compound endo-3c

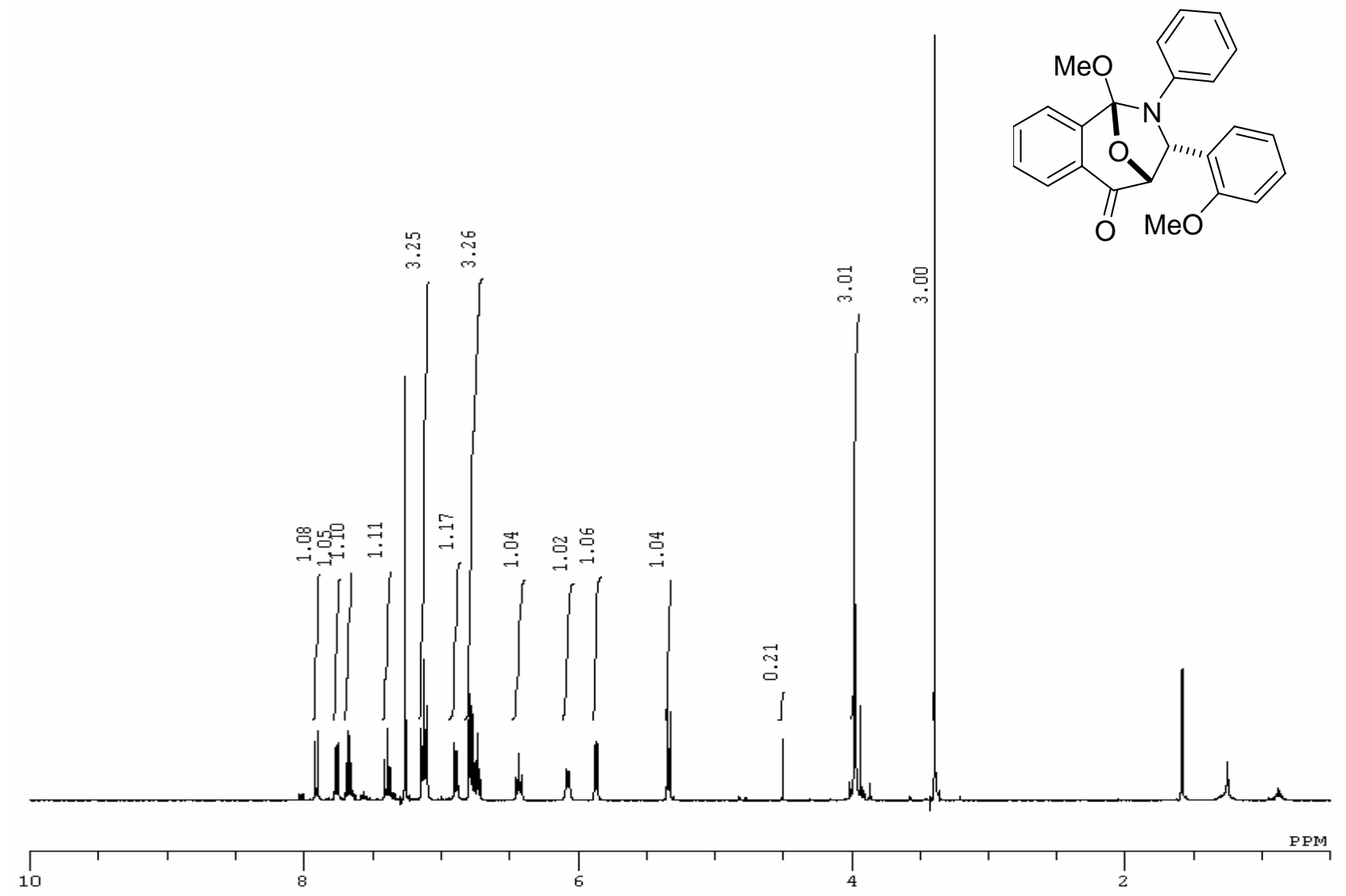

${ }^{13} \mathrm{C}$ NMR of Compound endo-3c
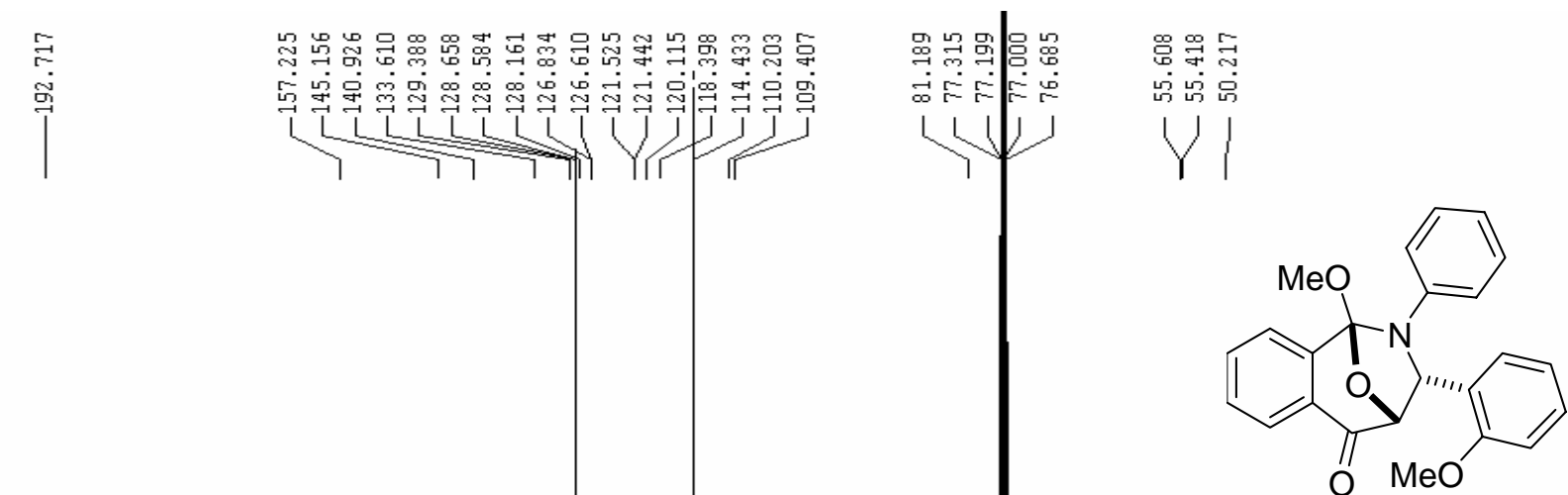
${ }^{1} \mathrm{H}$ NMR of Compound exo-3d

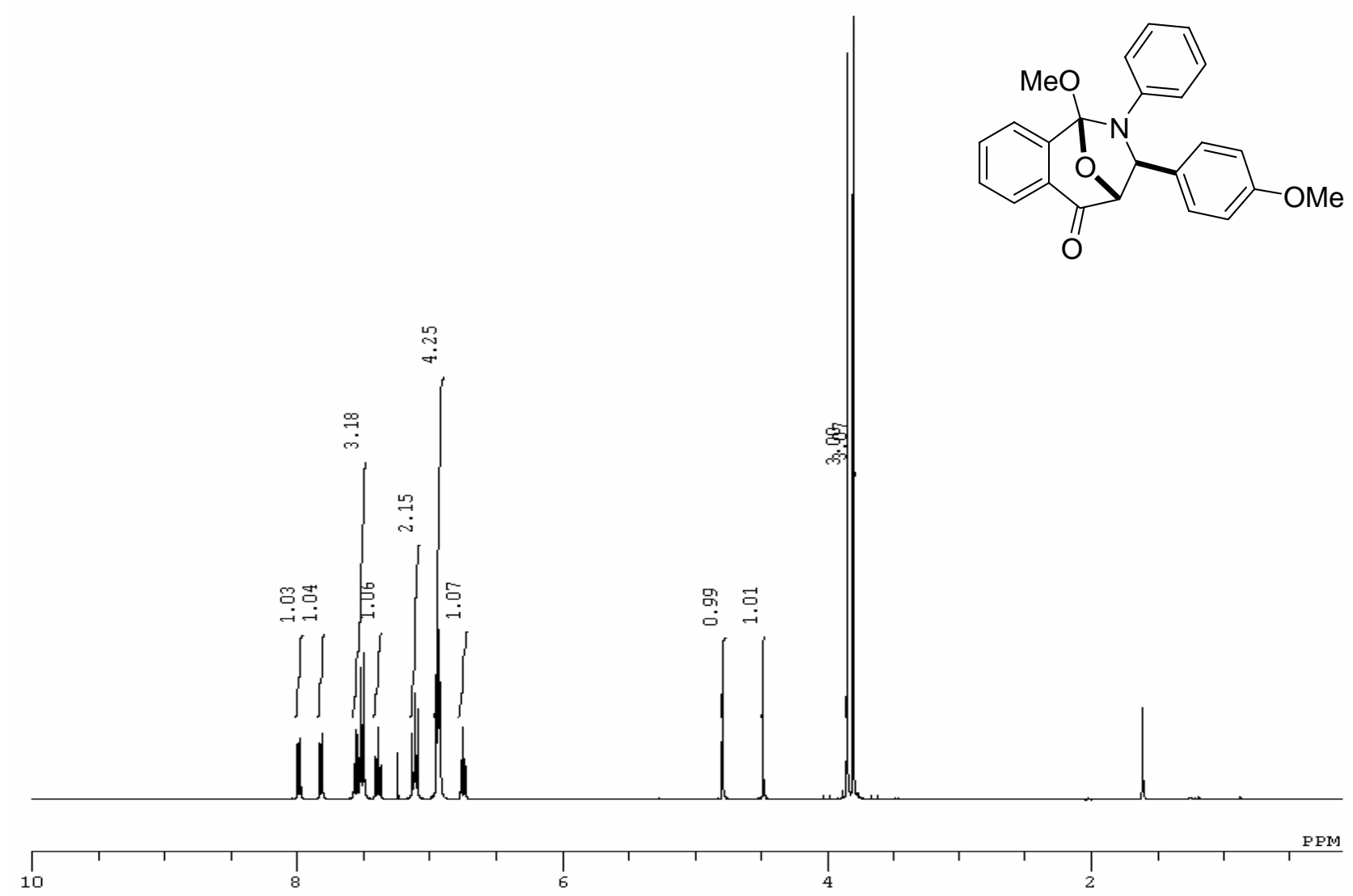

${ }^{13} \mathrm{C}$ NMR of Compound exo-3d

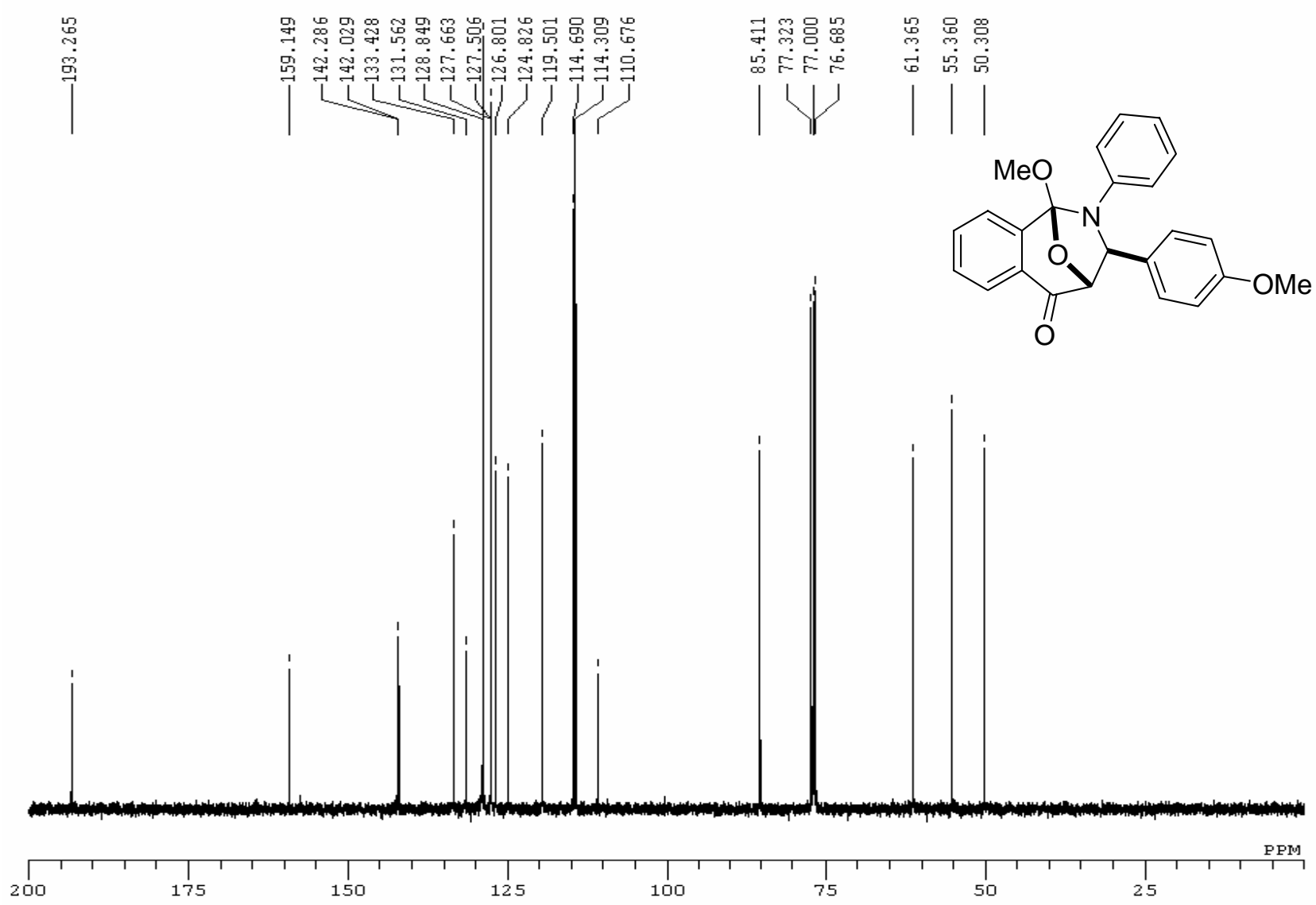


${ }^{1} \mathrm{H}$ NMR of Compound endo-3d

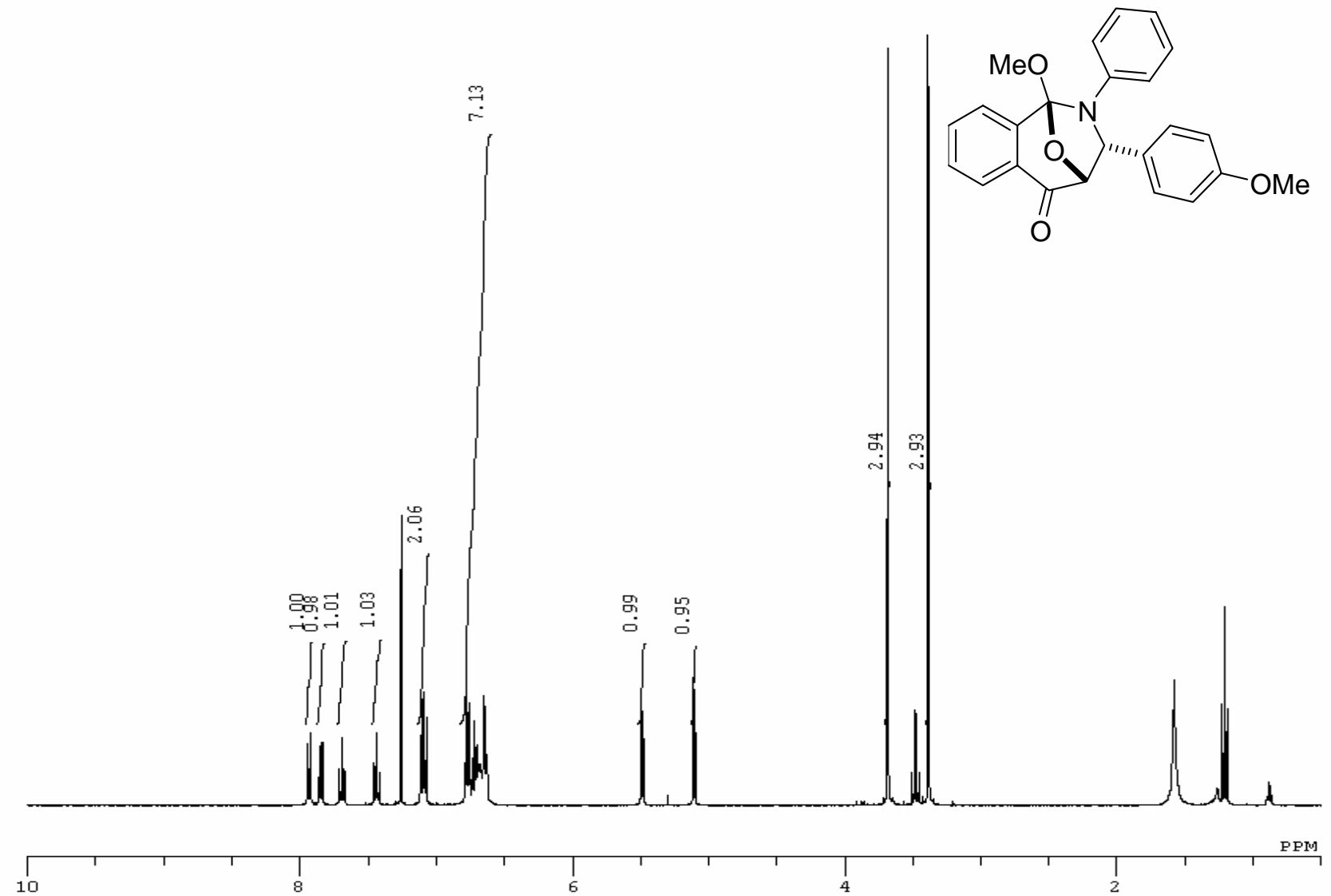

${ }^{13} \mathrm{C}$ NMR of Compound endo-3d

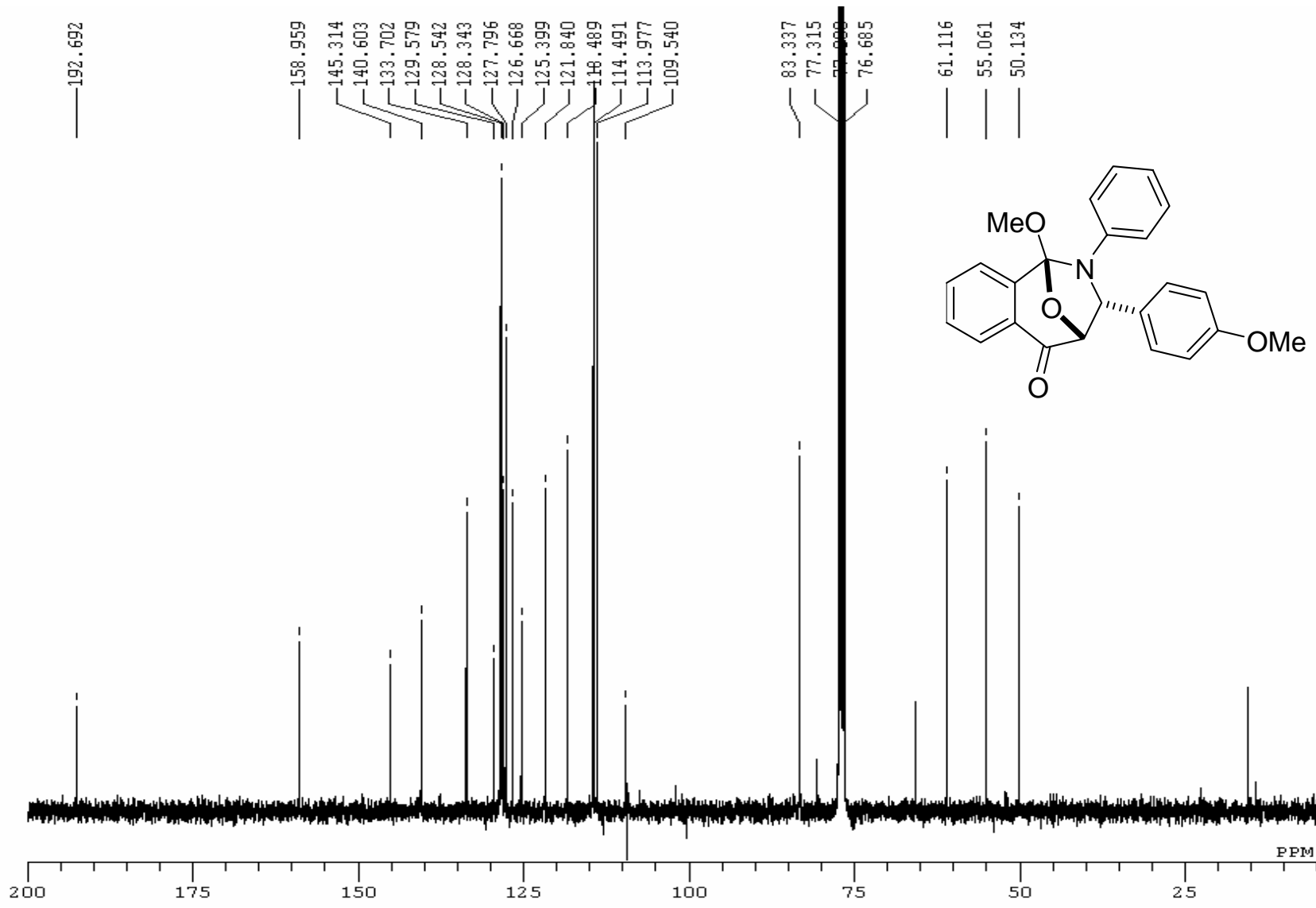


${ }^{1} \mathrm{H}$ NMR of Compound exo-3e
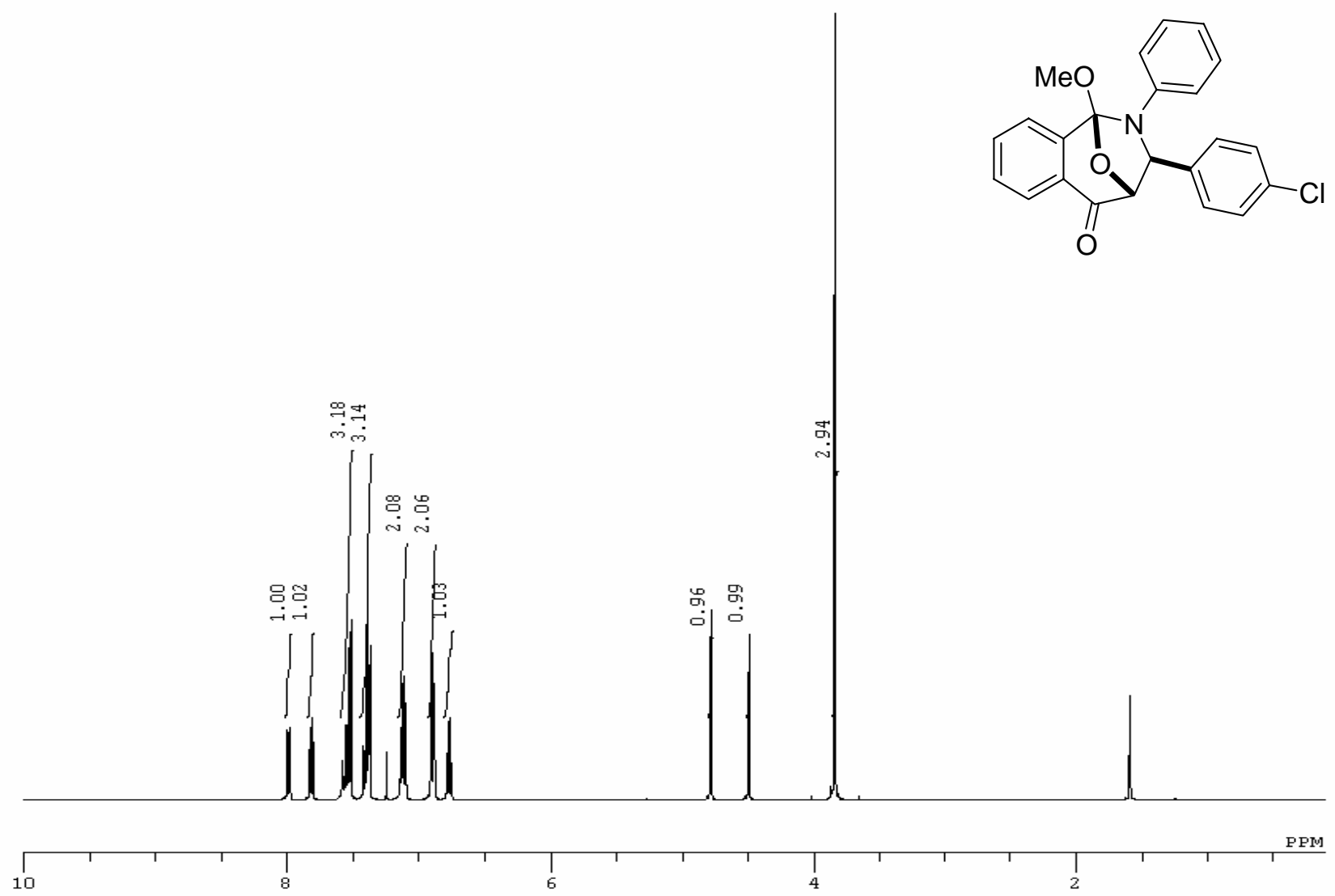

${ }^{13} \mathrm{C}$ NMR of Compound exo-3e

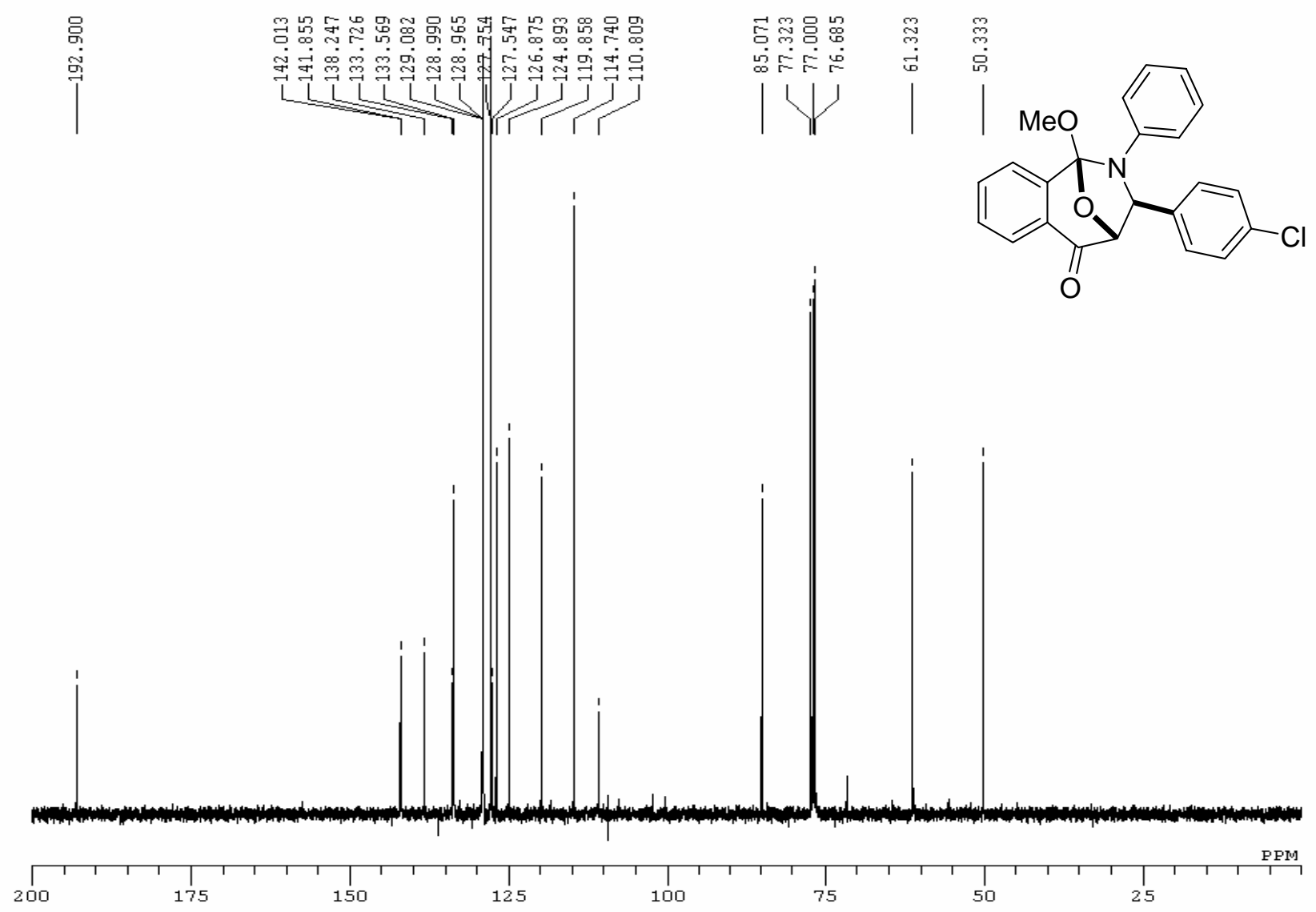


${ }^{1} \mathrm{H}$ NMR of Compound endo-3e

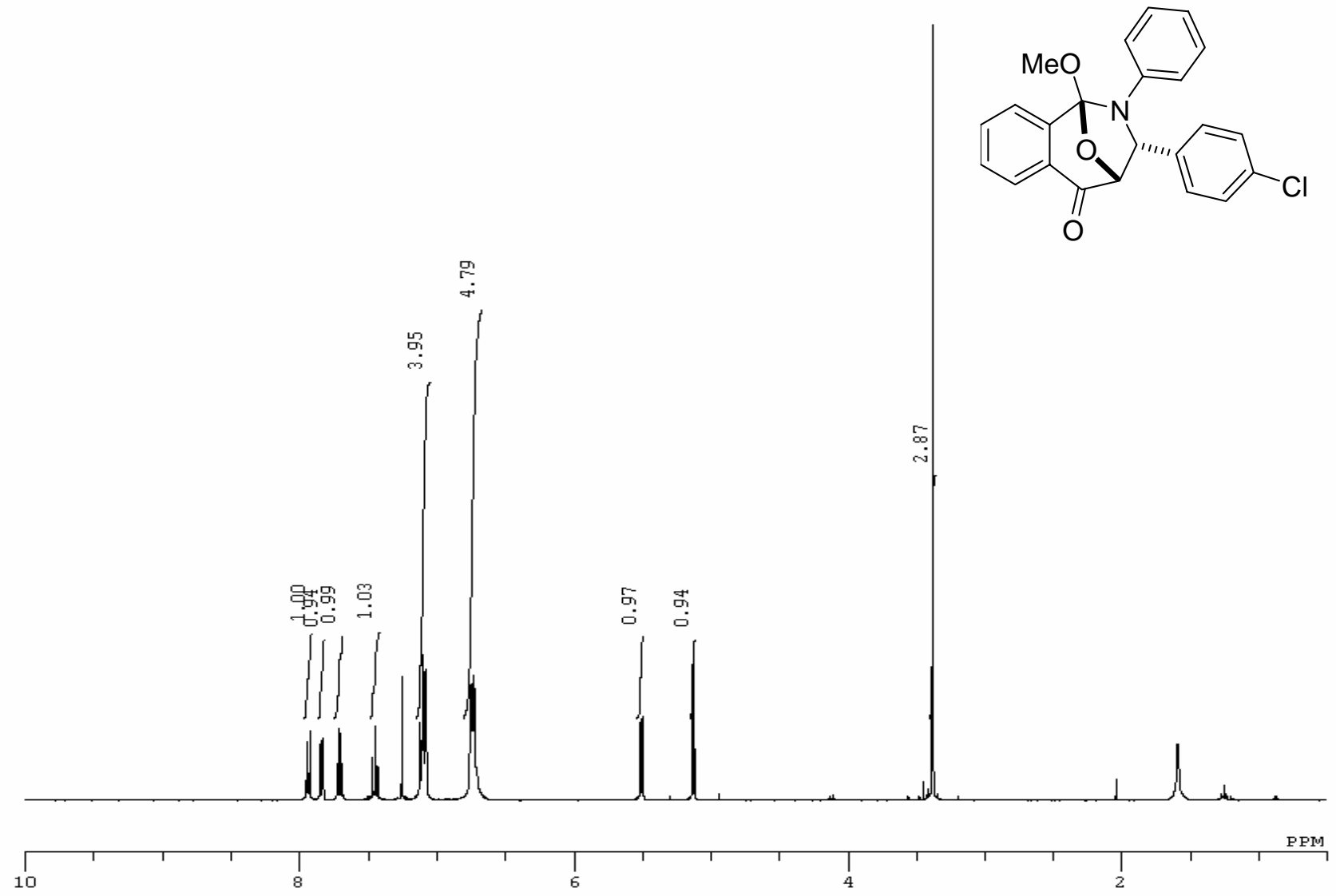

${ }^{13} \mathrm{C}$ NMR of Compound endo-3e

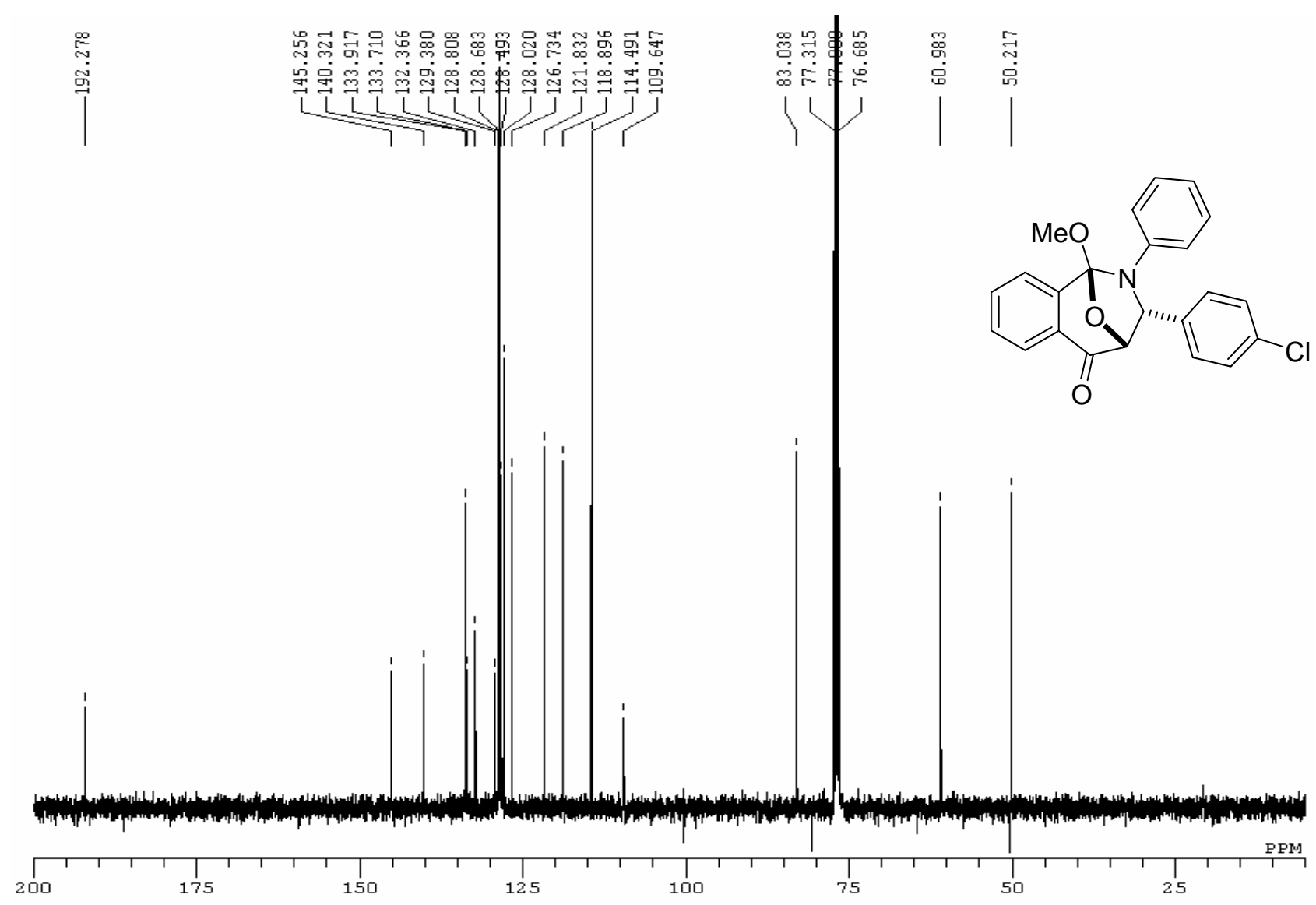


${ }^{1} \mathrm{H}$ NMR of Compound exo-3f
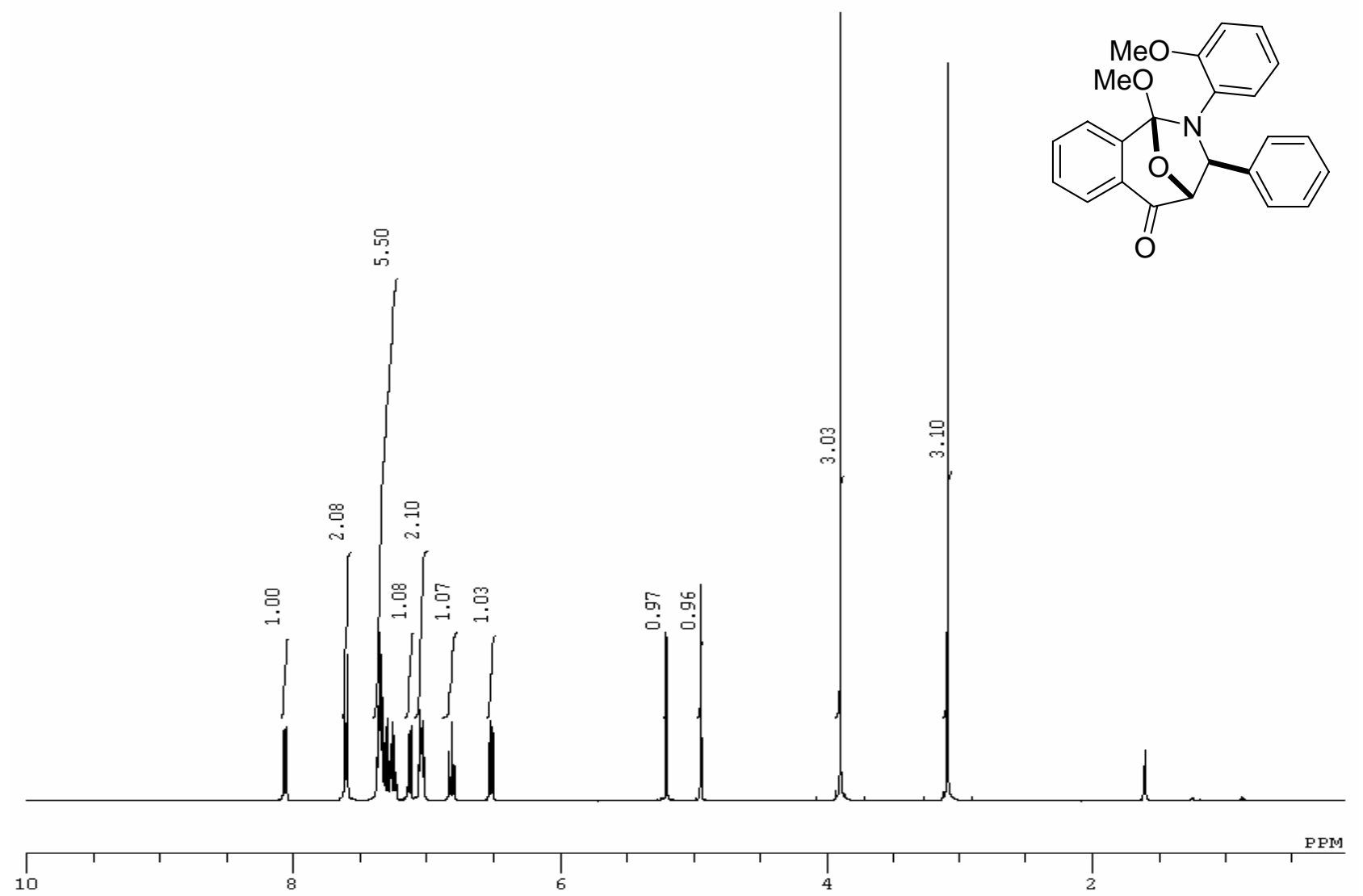

${ }^{13} \mathrm{C}$ NMR of Compound exo-3f

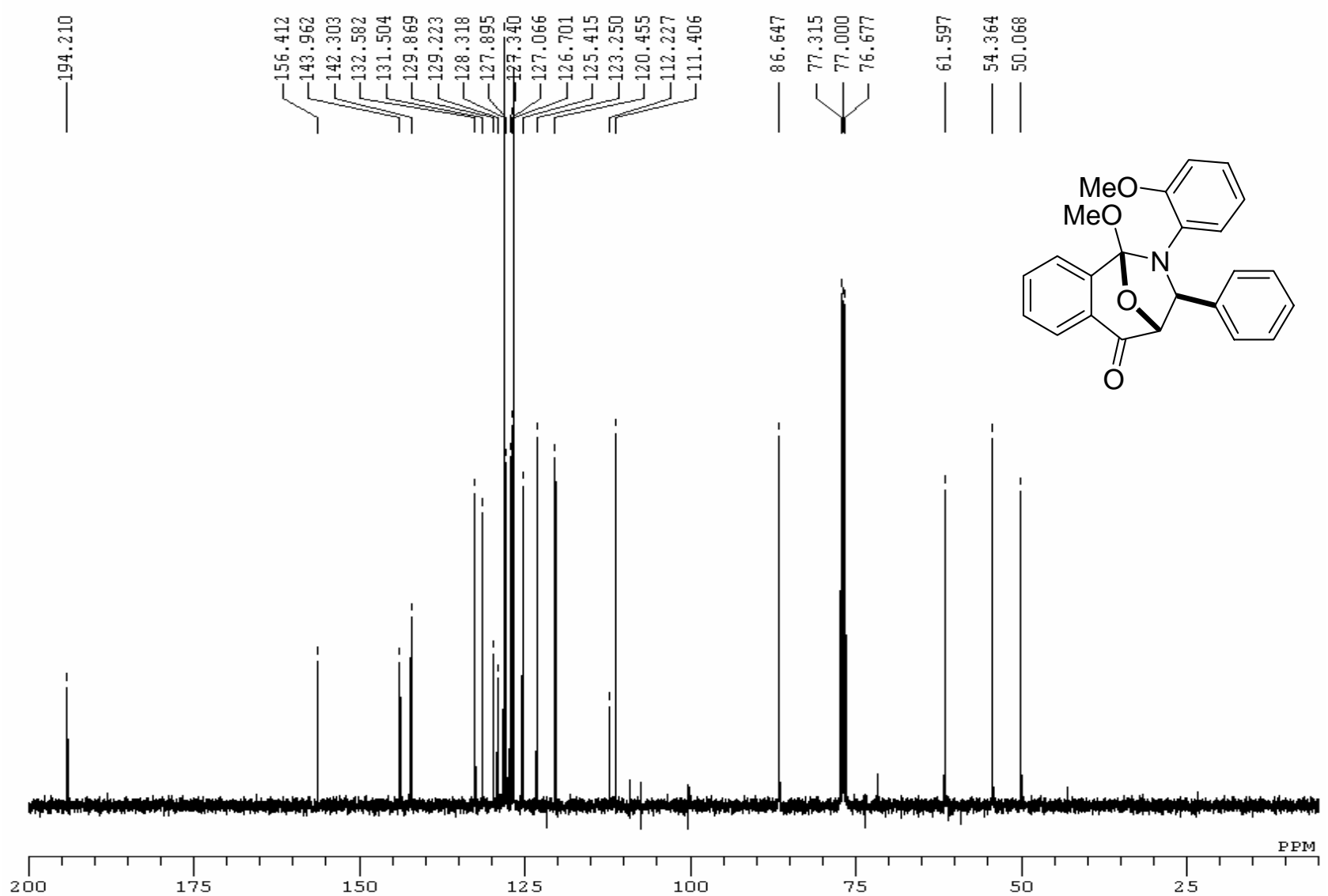


${ }^{1} \mathrm{H}$ NMR of Compound endo-3f

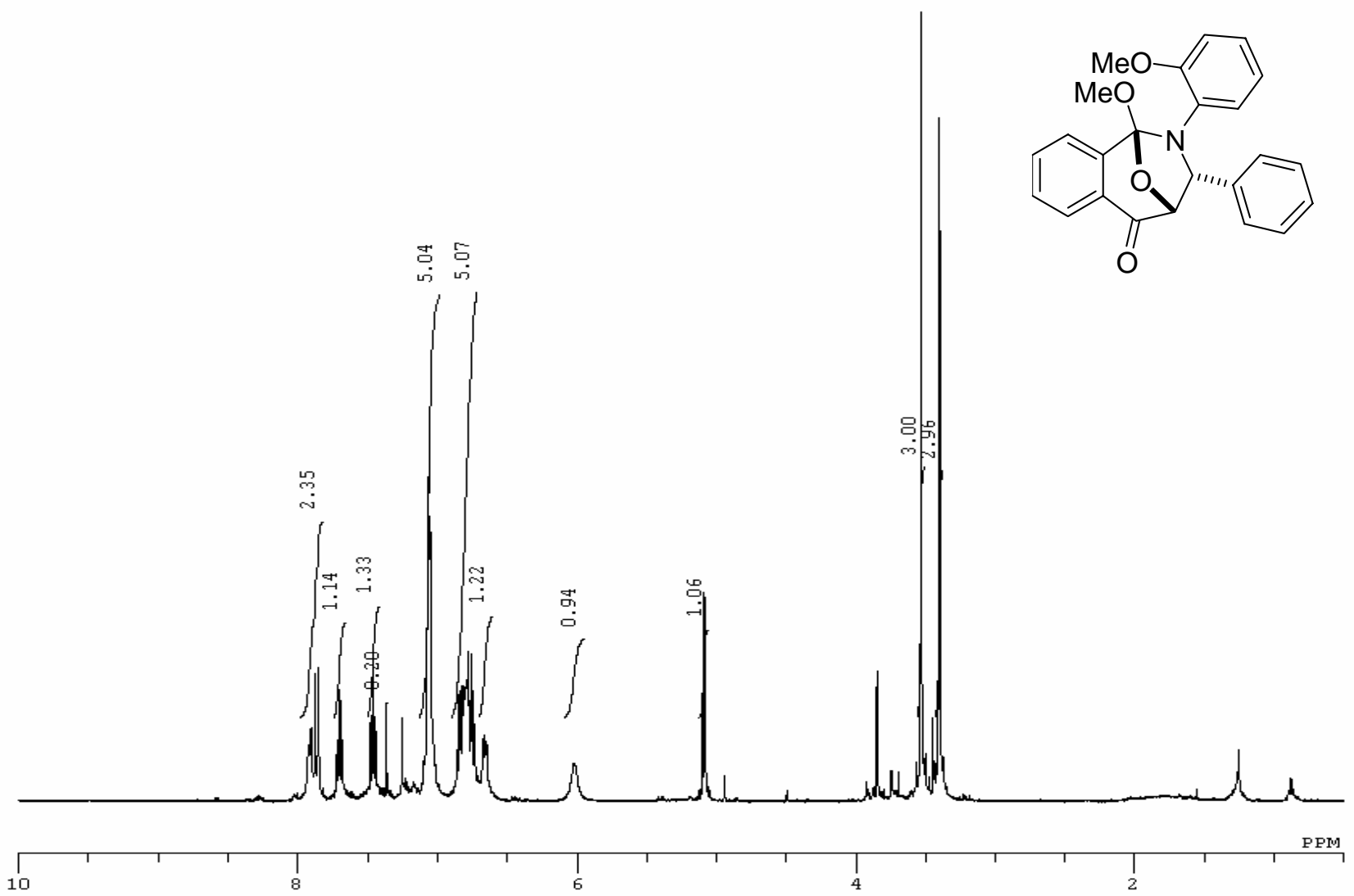

${ }^{1} \mathrm{H}$ NMR of Compound exo-3g

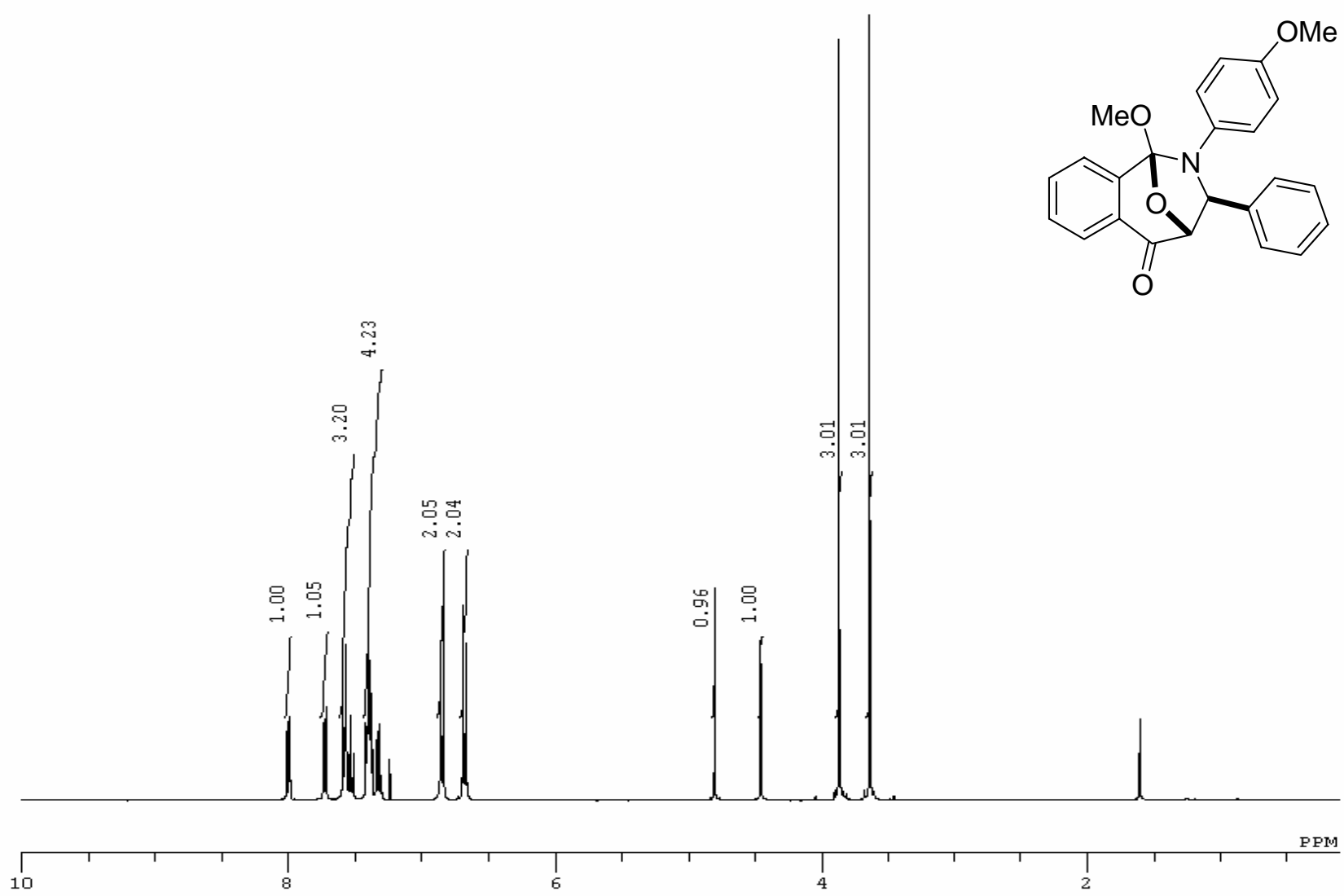


${ }^{13} \mathrm{C}$ NMR of Compound exo-3g

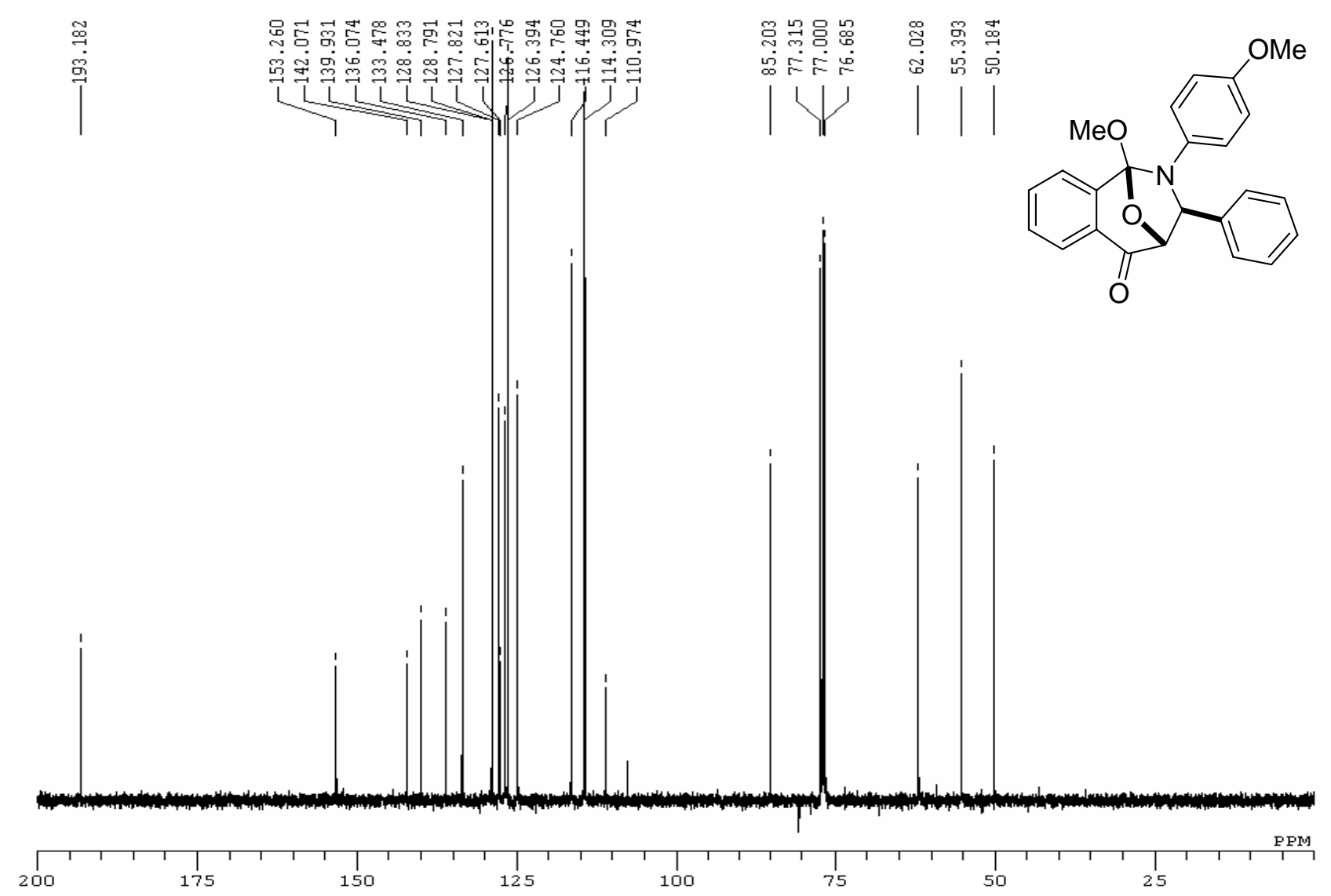

${ }^{1} \mathrm{H}$ NMR of Compound endo-3g

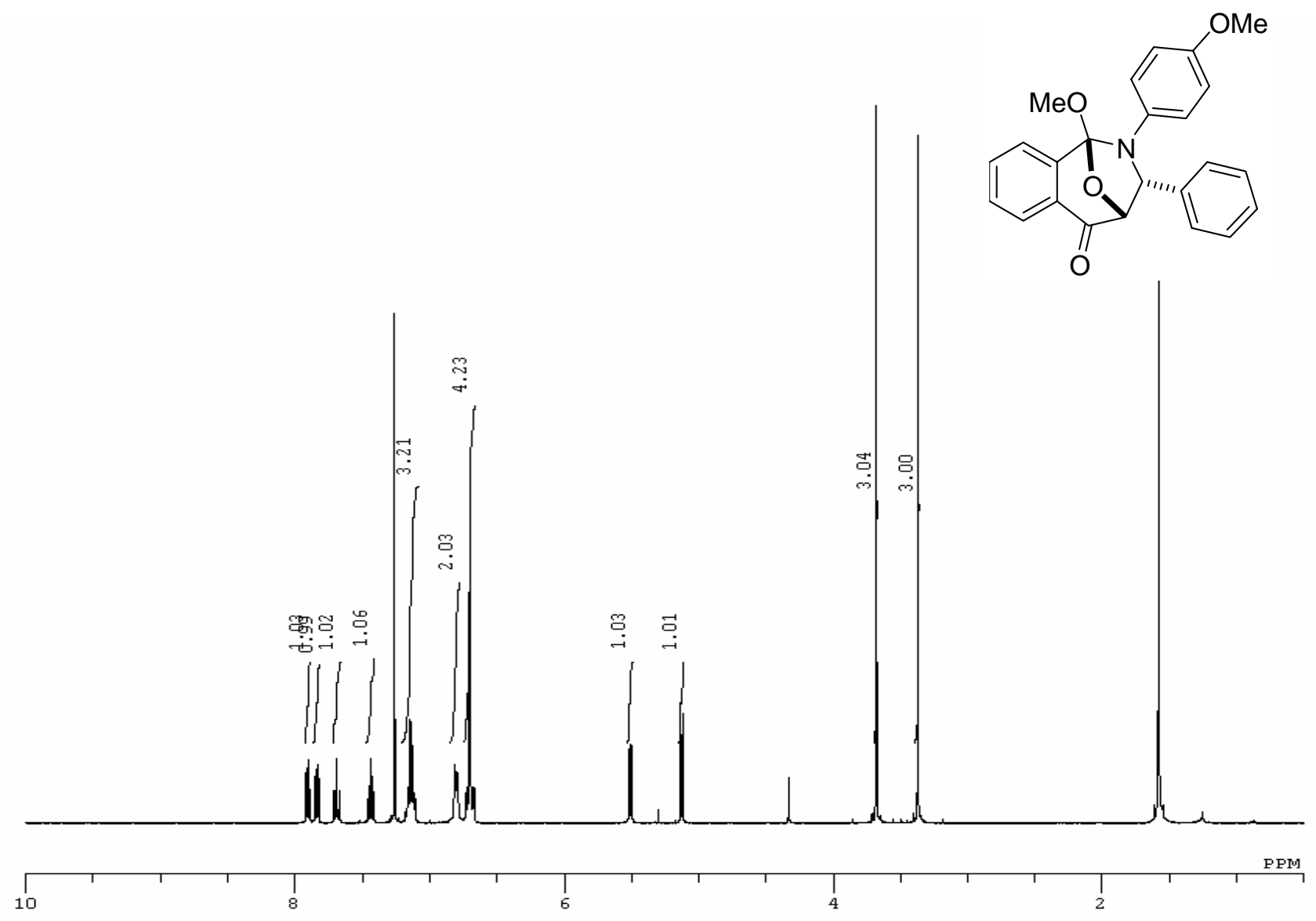


${ }^{1} \mathrm{H}$ NMR of Compound exo-3h

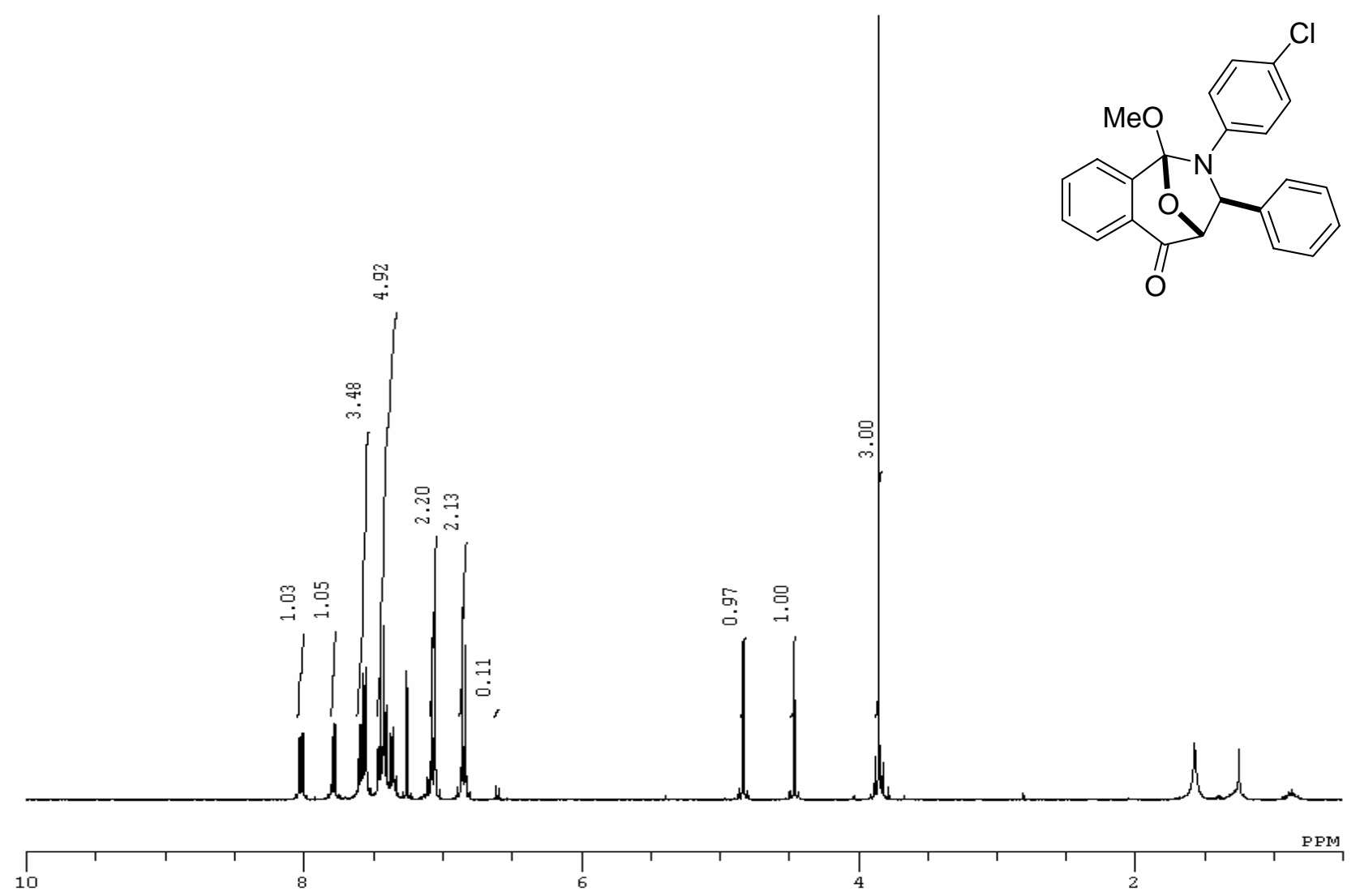

${ }^{13} \mathrm{C}$ NMR of Compound exo-3h

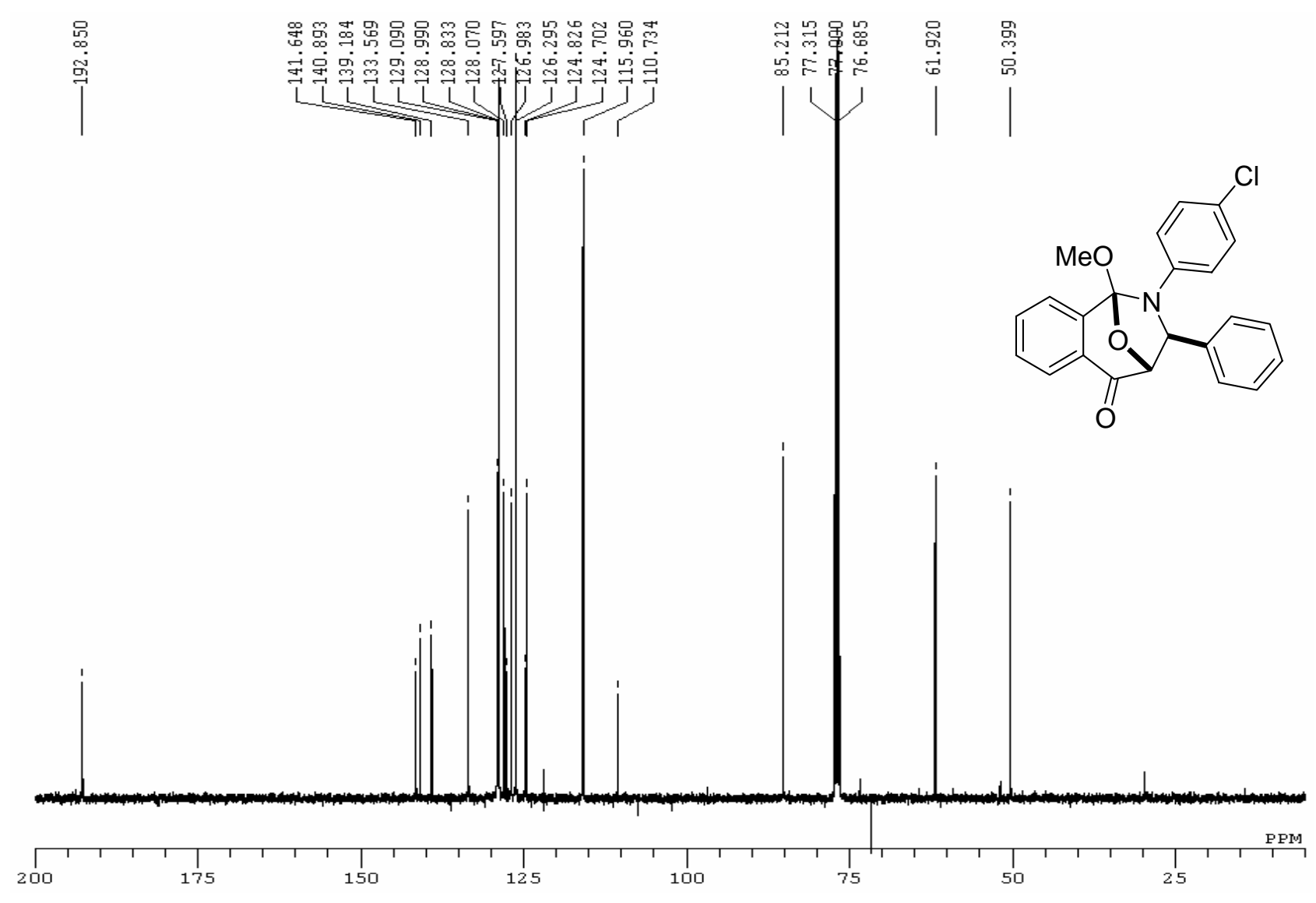


${ }^{1}$ H NMR of Compound exo-3i
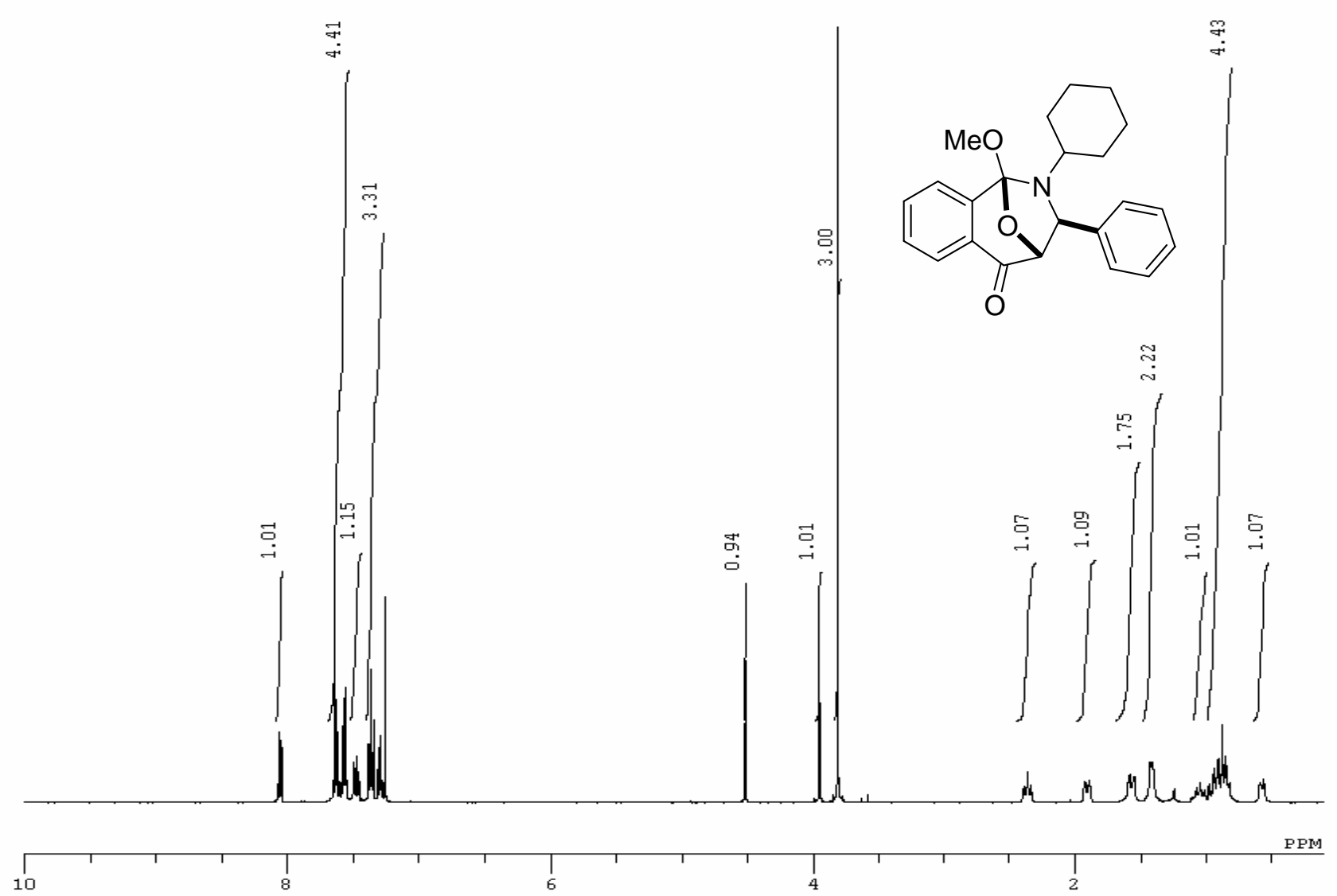

${ }^{13} \mathrm{C}$ NMR of Compound exo-3i

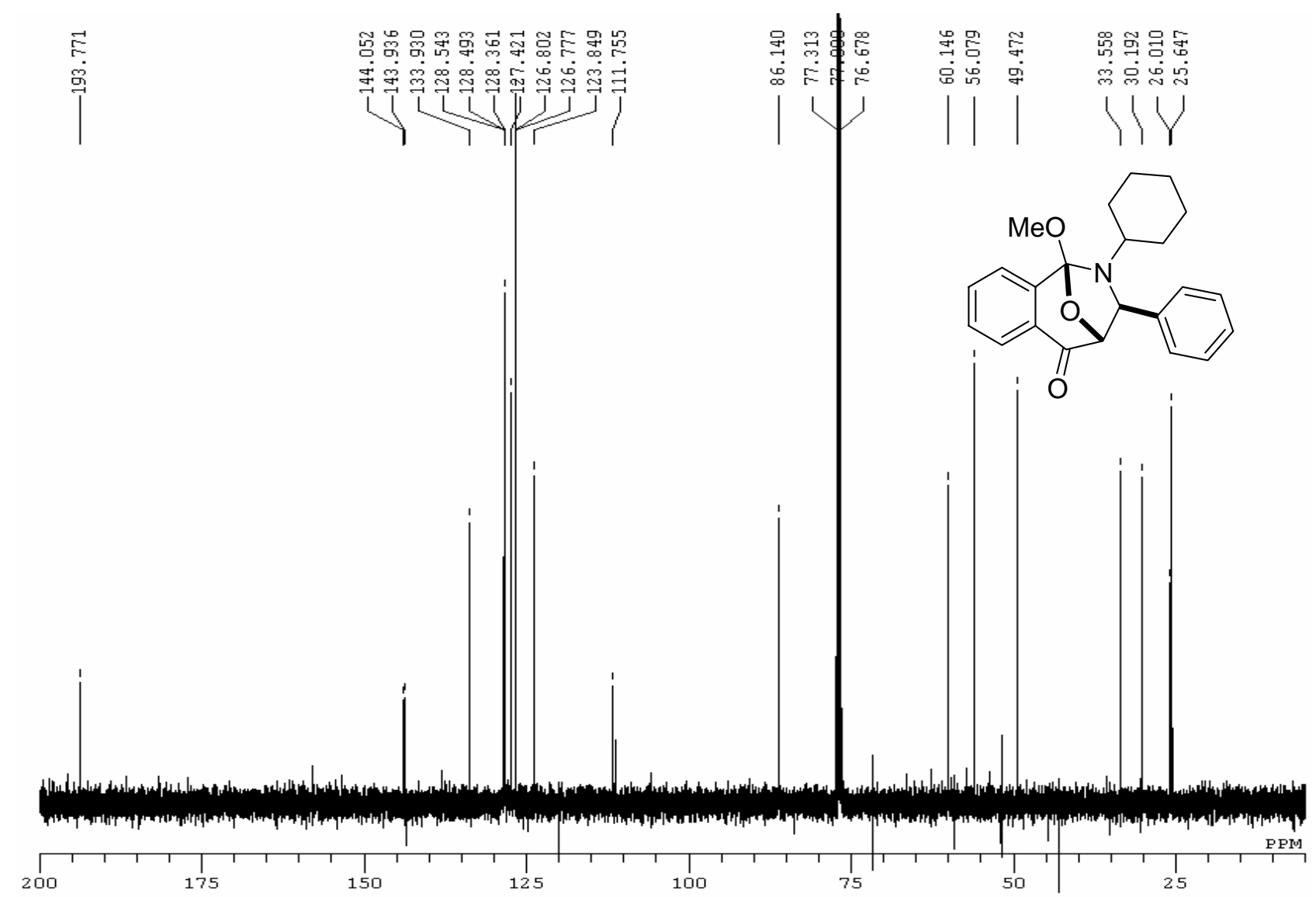


${ }^{1} \mathrm{H}$ NMR of Compound endo-3i

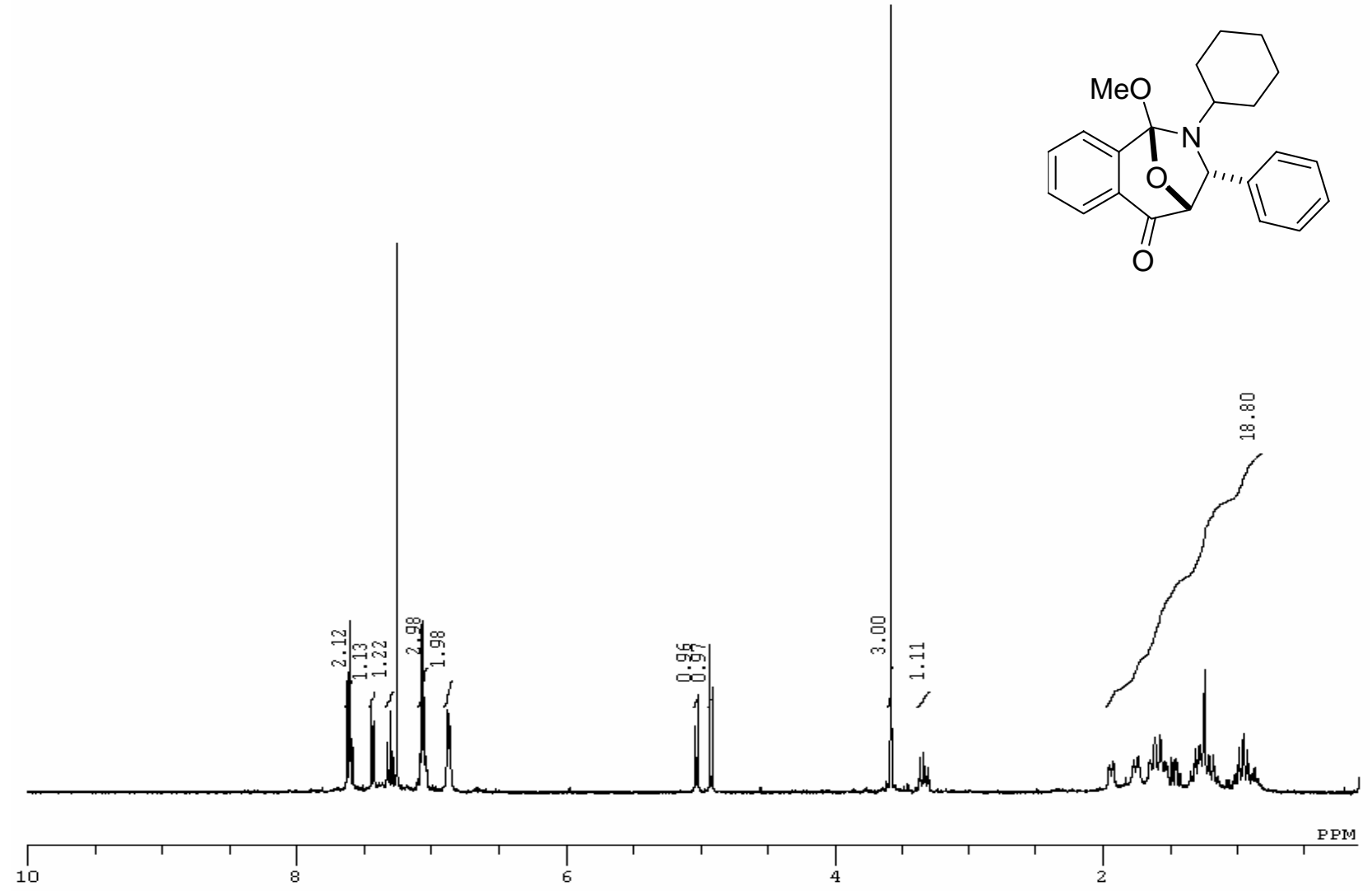

${ }^{1} \mathrm{H}$ NMR of Compound exo-3j

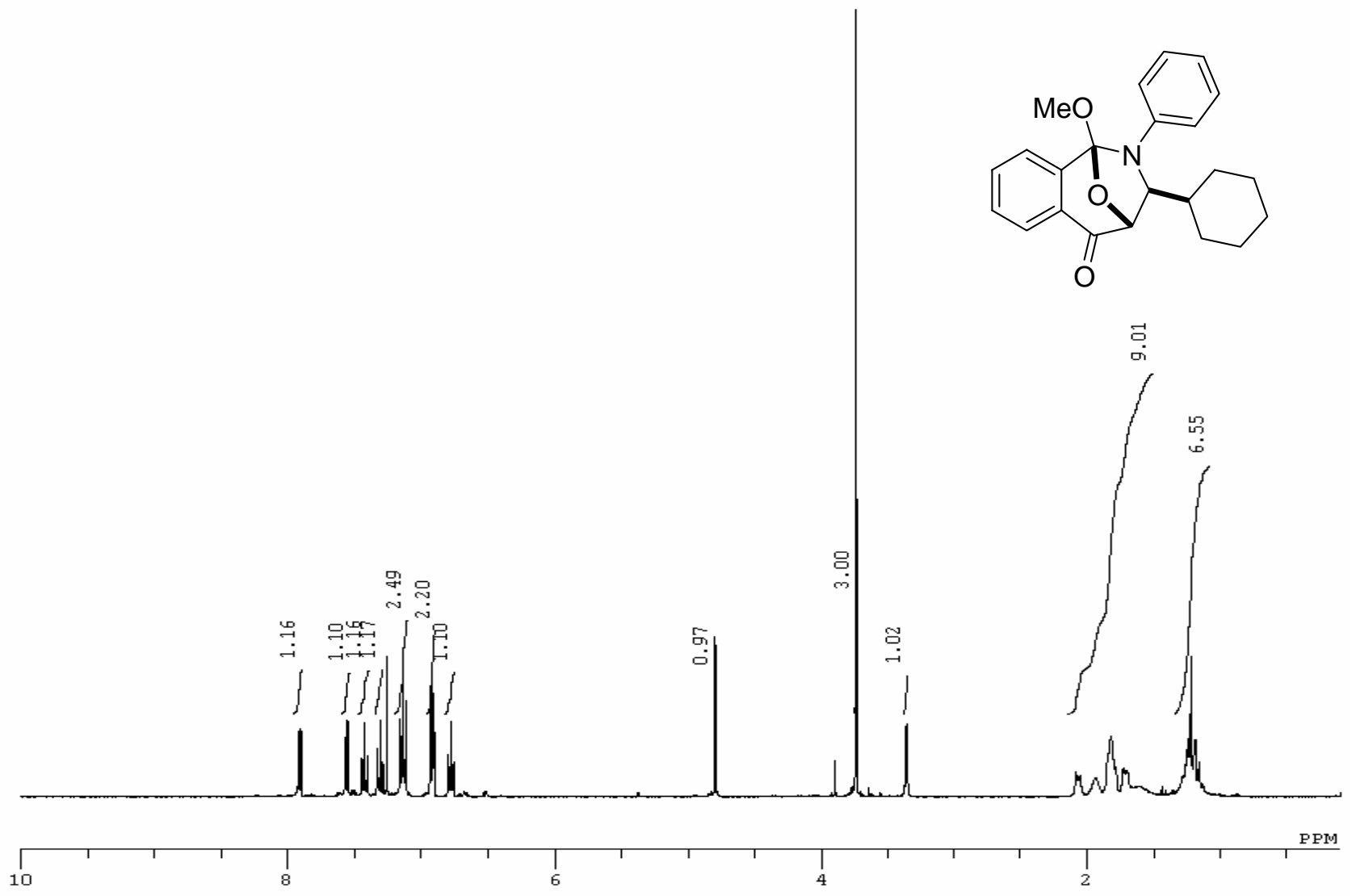


${ }^{13} \mathrm{C}$ NMR of Compound exo-3j

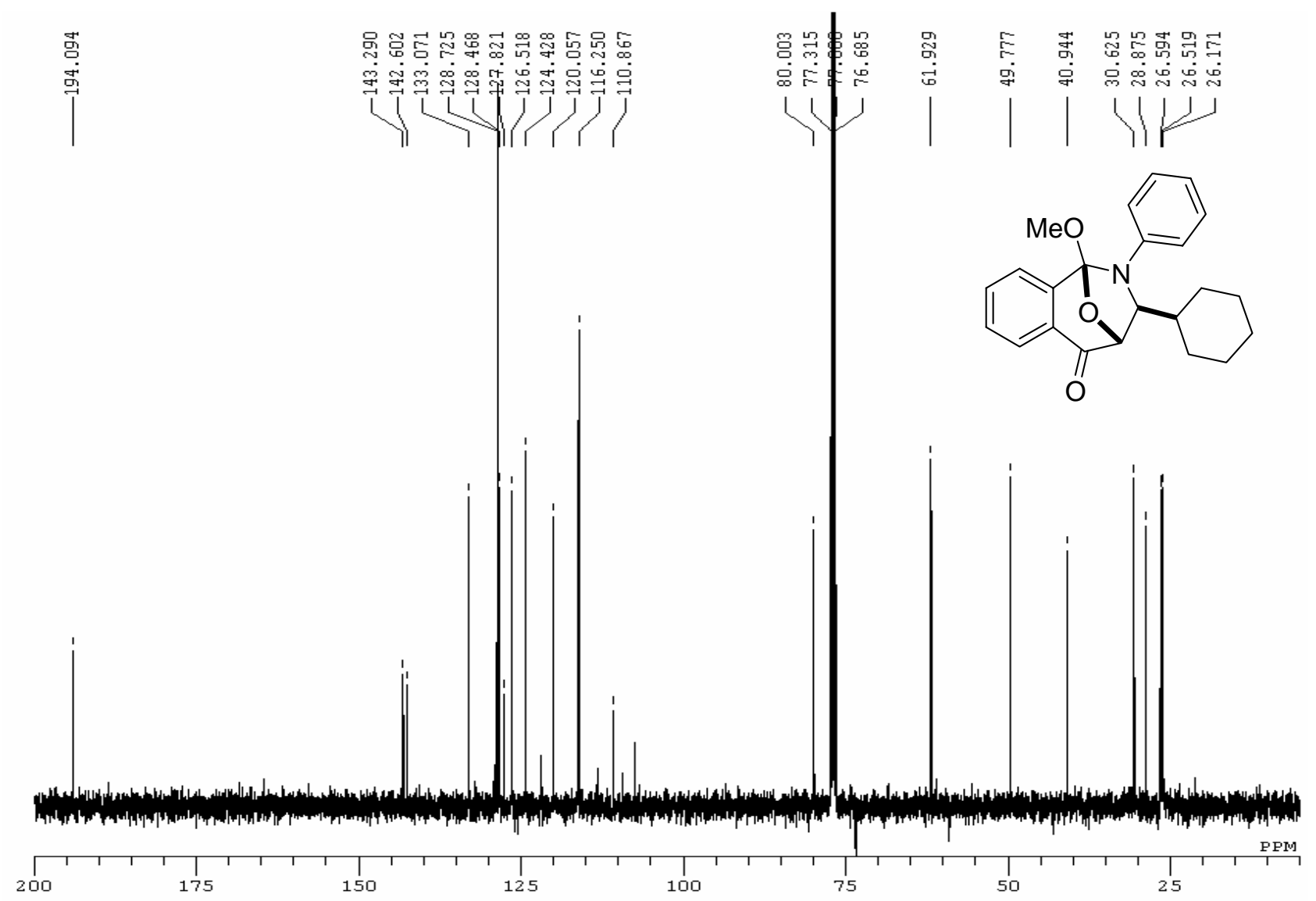

${ }^{1} \mathrm{H}$ NMR of Compound endo-3k

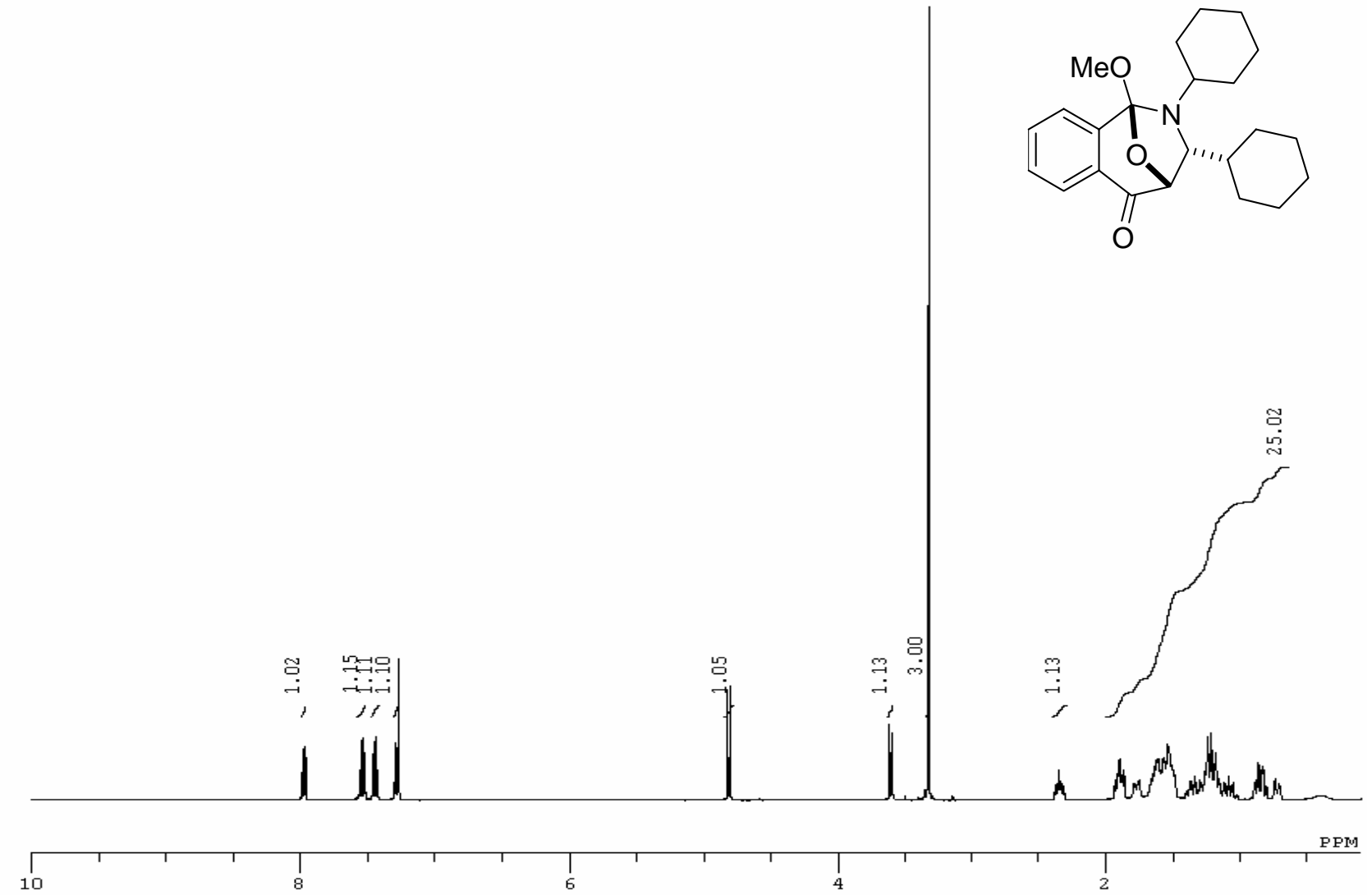


${ }^{13} \mathrm{C}$ NMR of Compound endo-3k

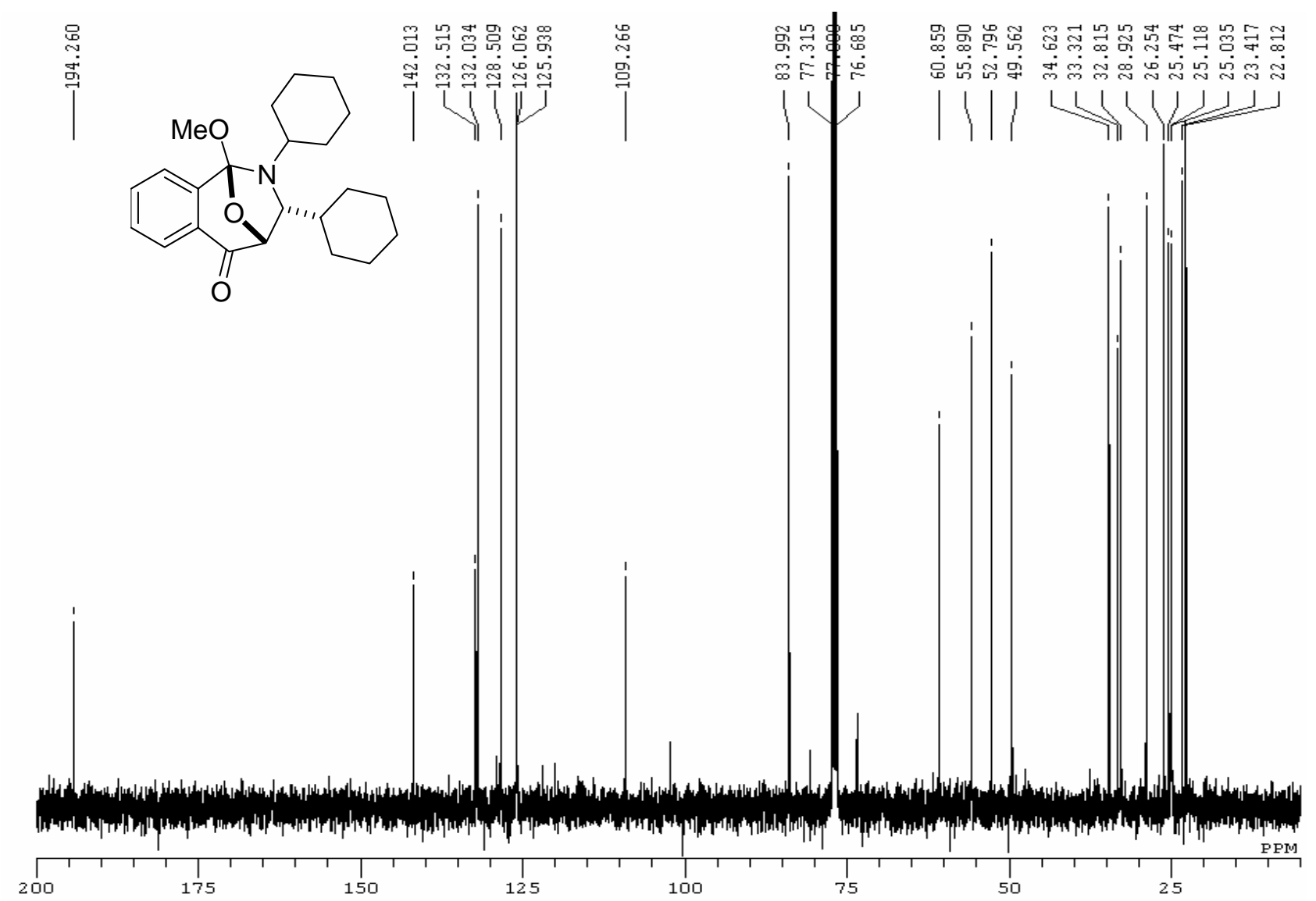

${ }^{1} \mathrm{H}$ NMR of Compound exo-31

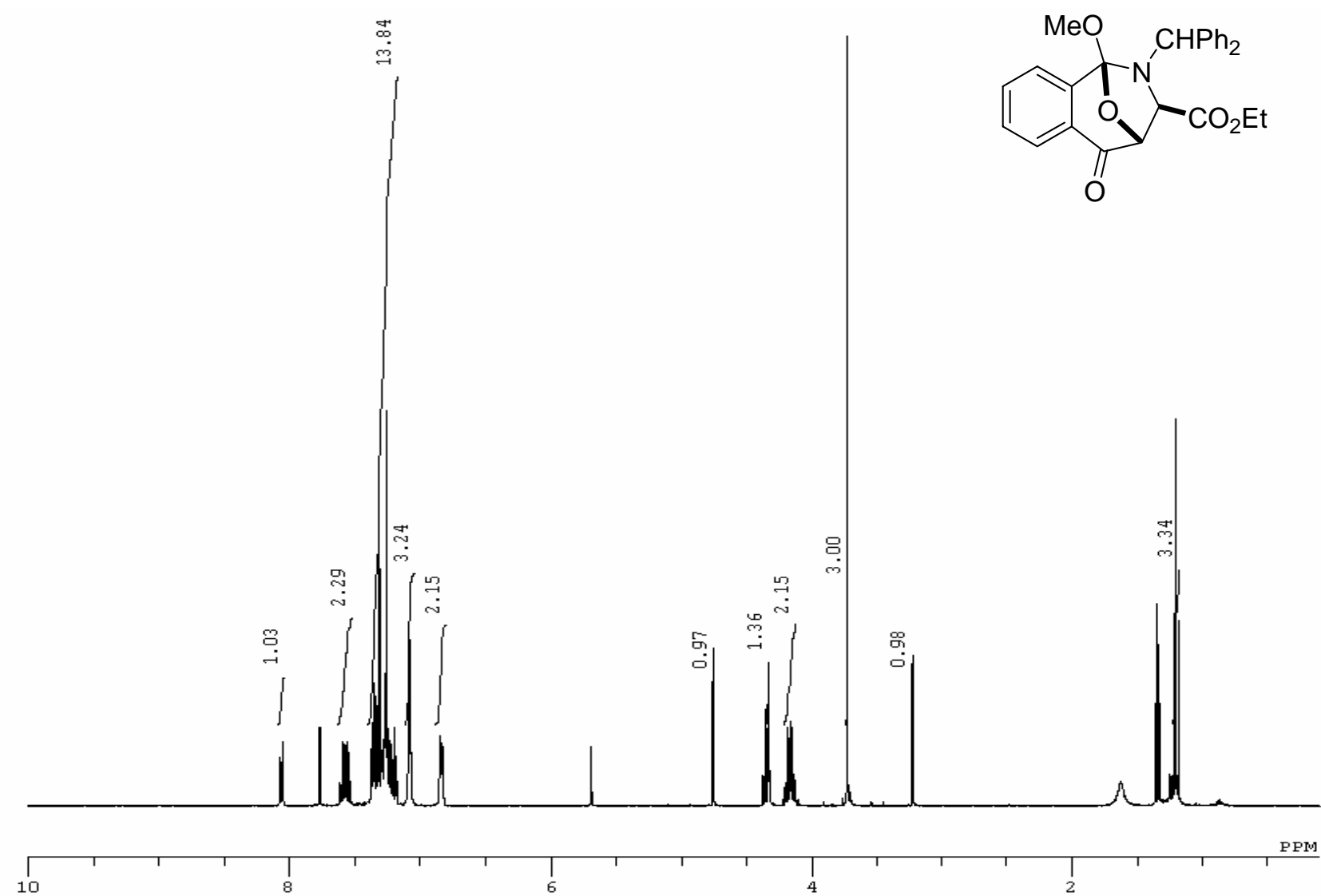


${ }^{1} \mathrm{H}$ NMR of Compound endo-31

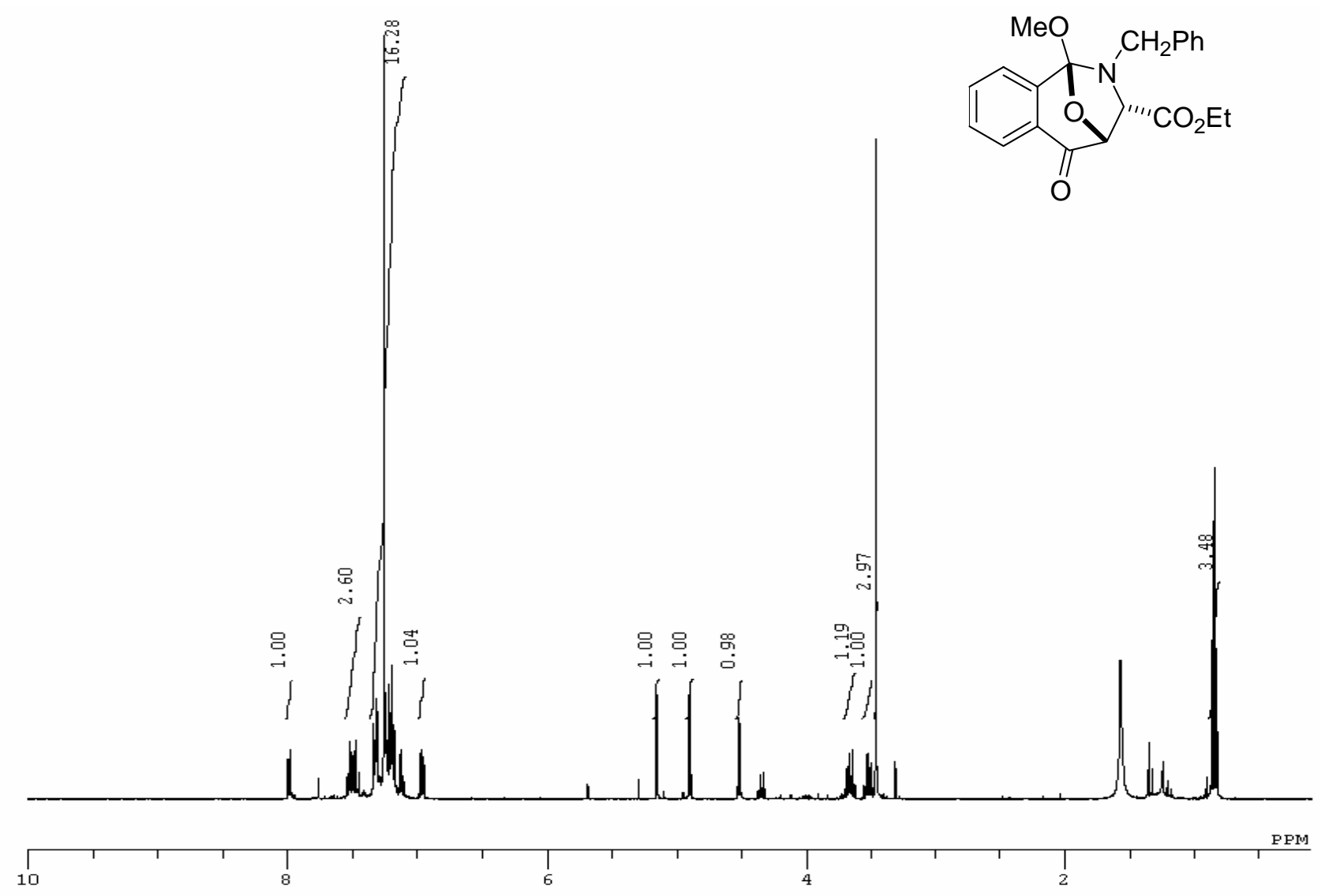


${ }^{1} \mathrm{H}$ NMR of Compound exo-6c

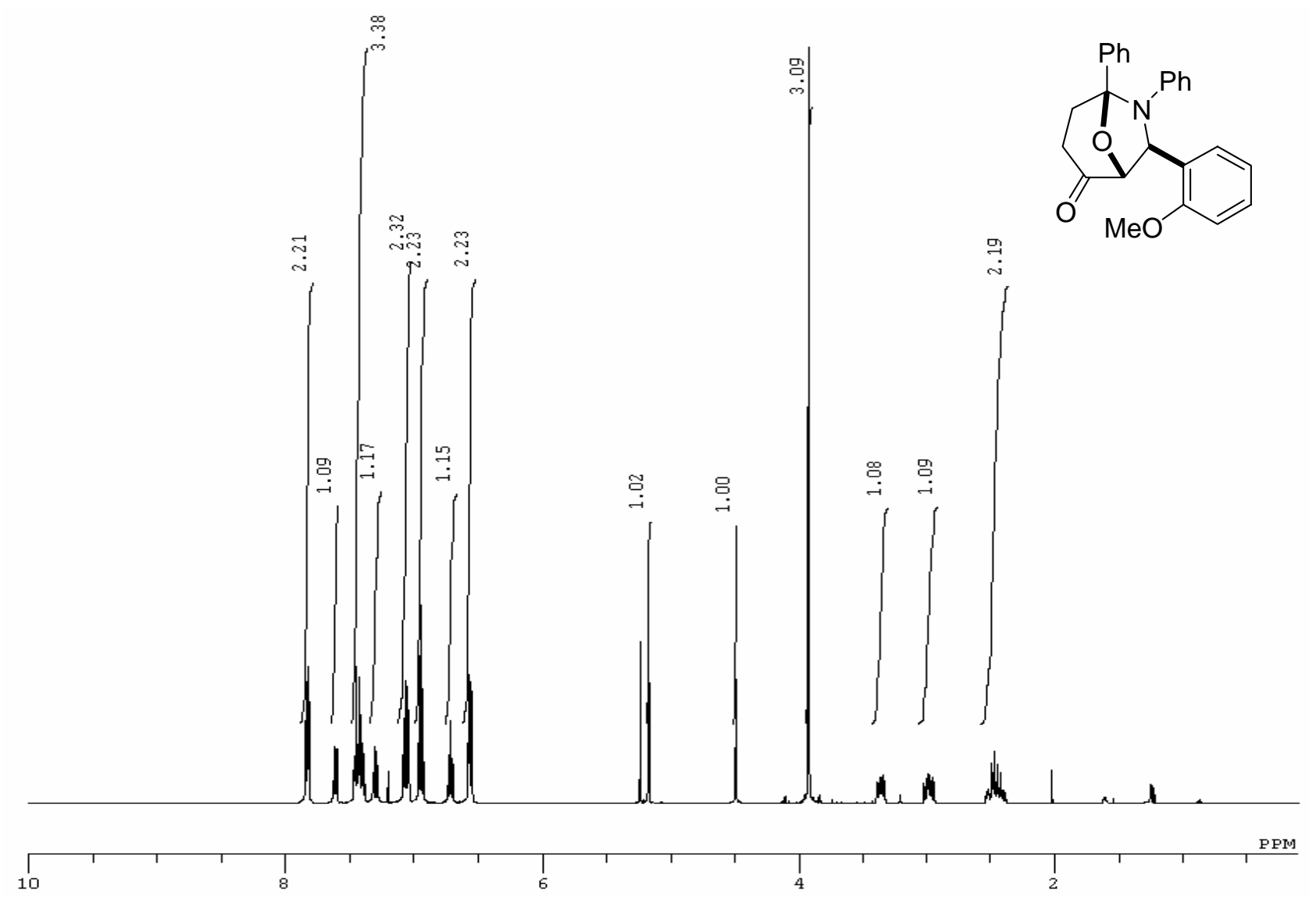

${ }^{13} \mathrm{C}$ NMR of Compound exo-6c

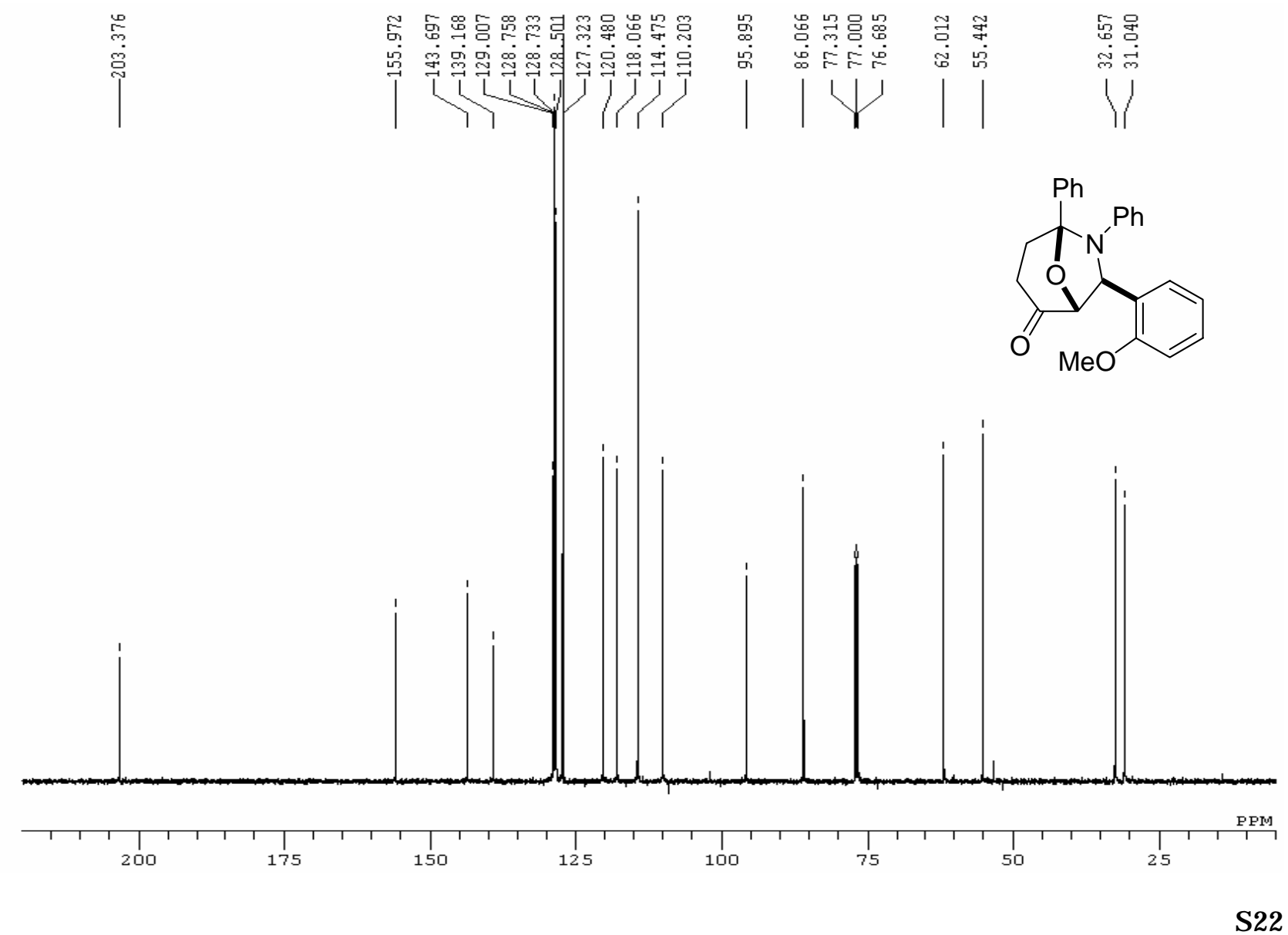


${ }^{1} \mathrm{H}$ NMR of Compound endo-6c

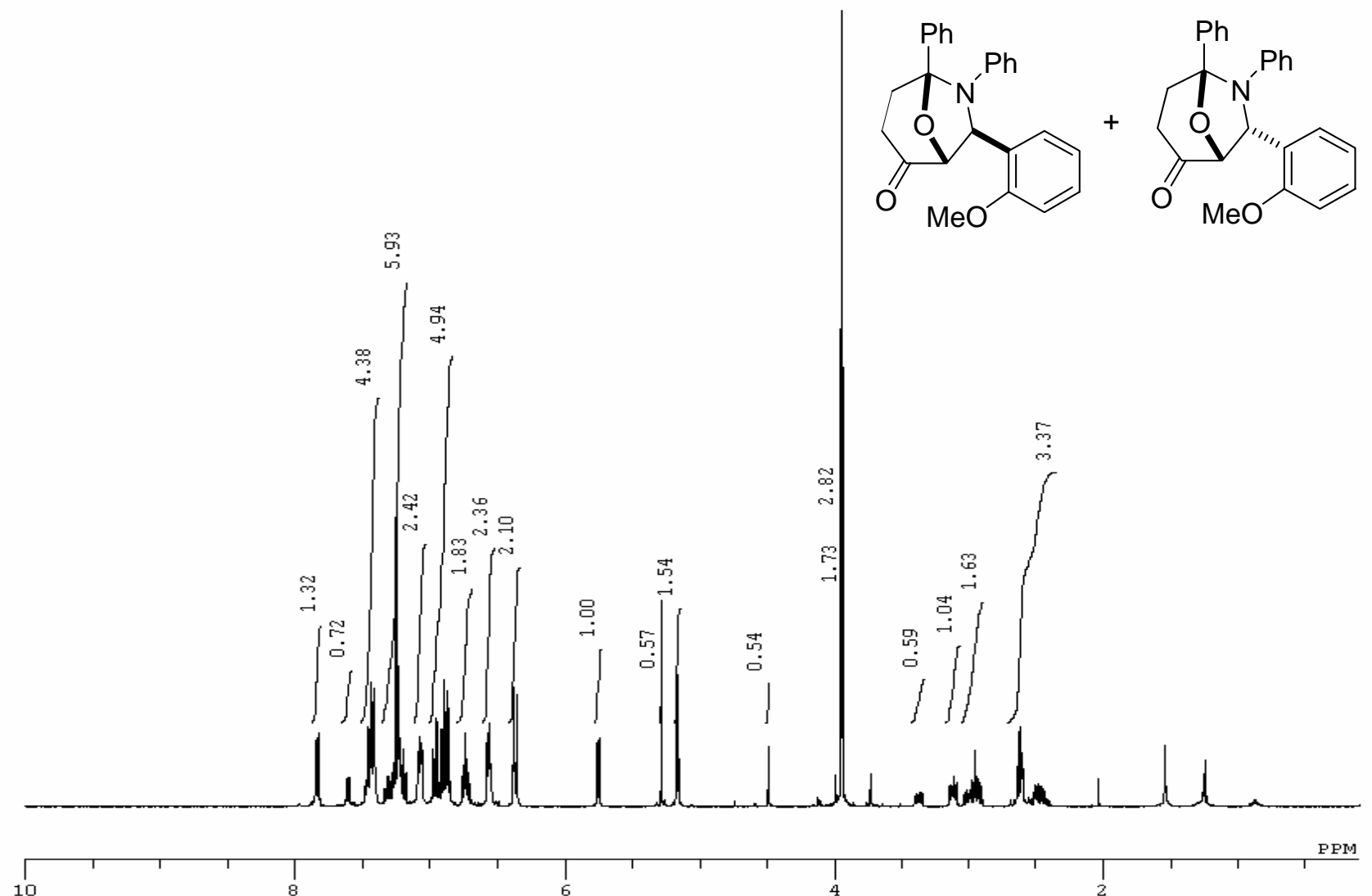

${ }^{13} \mathrm{C}$ NMR of Compound endo-6c

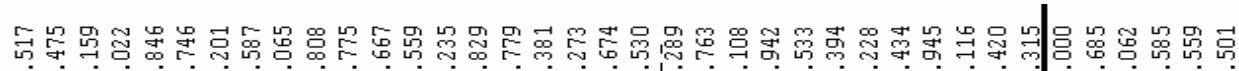

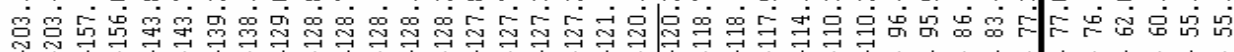

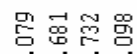

ज्ञां क्षित्र

$1+$

$11 Y Y$

(1/<smiles>COc1ccccc1[C@@H]1[C@H]2OC(c3ccccc3)(CCC2=O)N(c2ccccc2)[C@H]1c1ccccc1OC</smiles>

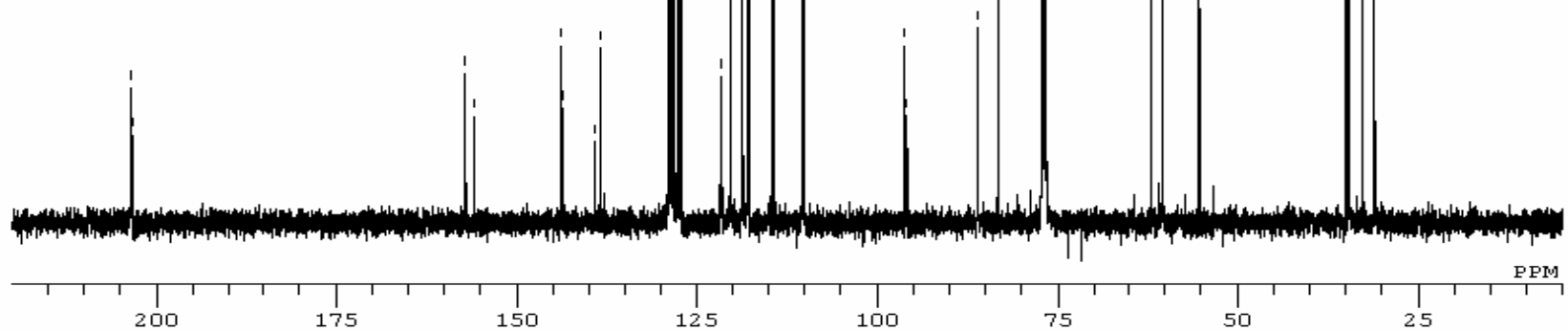


${ }^{1} \mathrm{H}$ NMR of Compound exo-9c
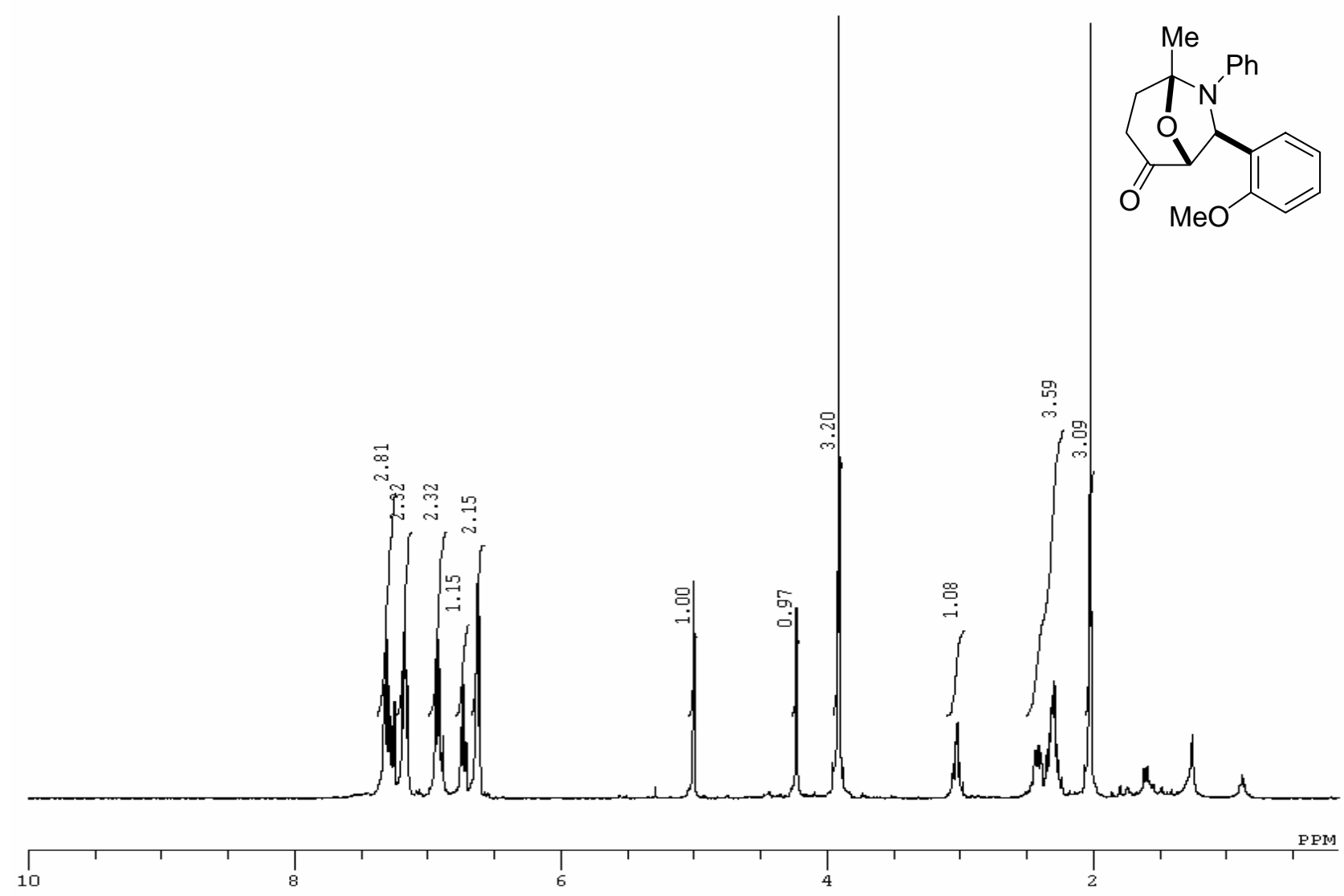

${ }^{13} \mathrm{C}$ NMR of Compound exo-9c

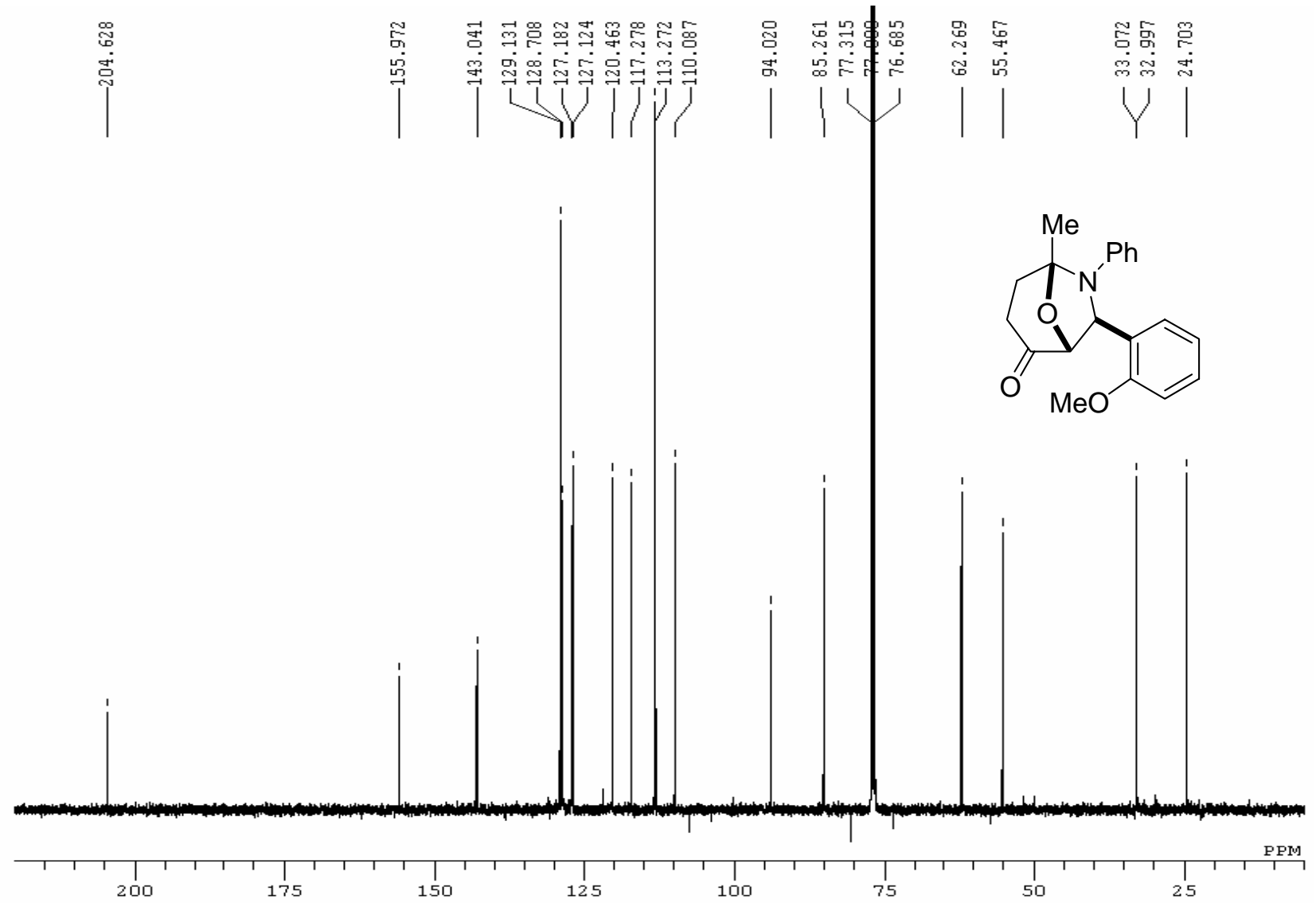


${ }^{1} \mathrm{H}$ NMR of Compound endo-9c

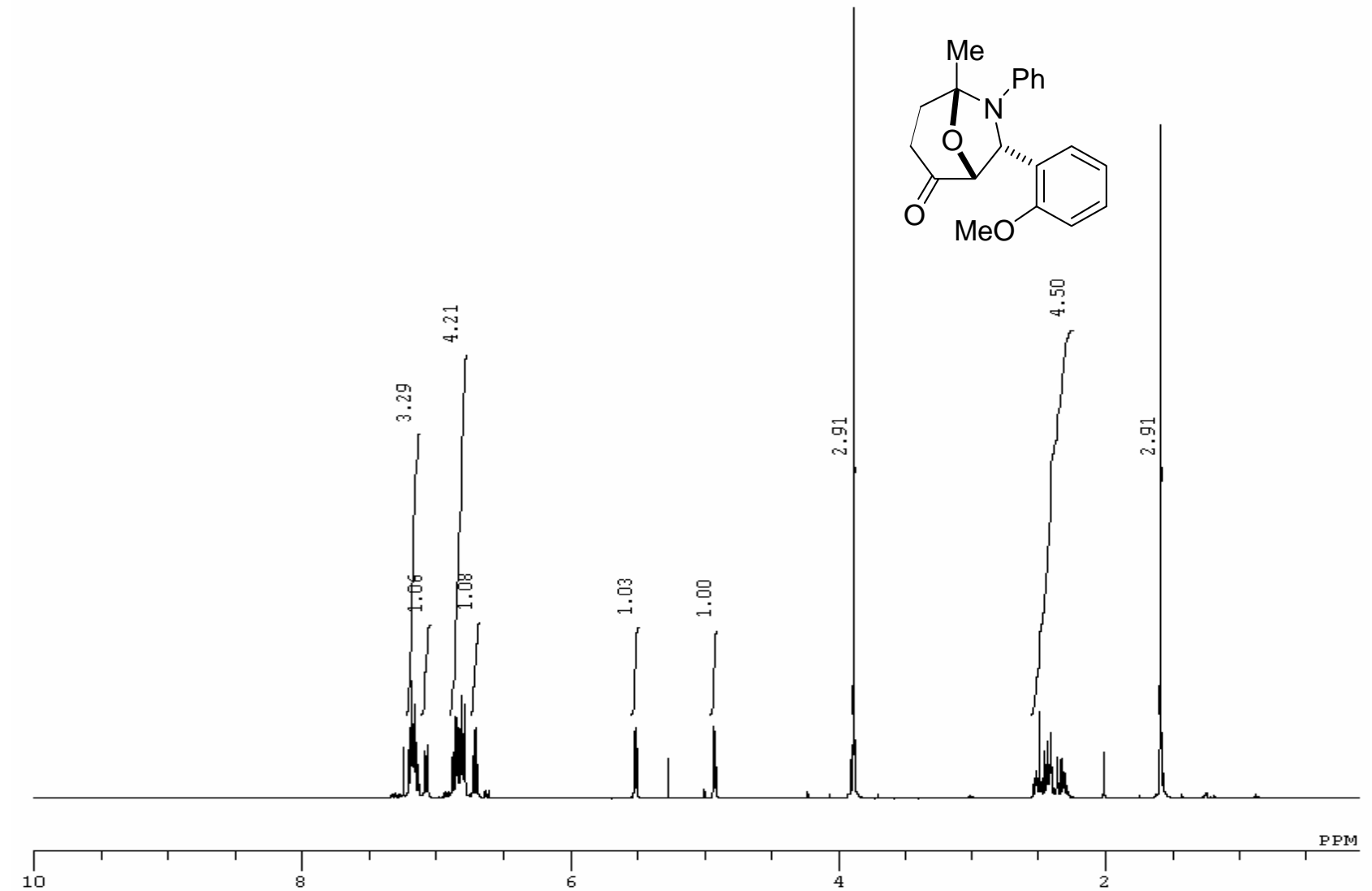

${ }^{13} \mathrm{C}$ NMR of Compound endo-9c

| | |

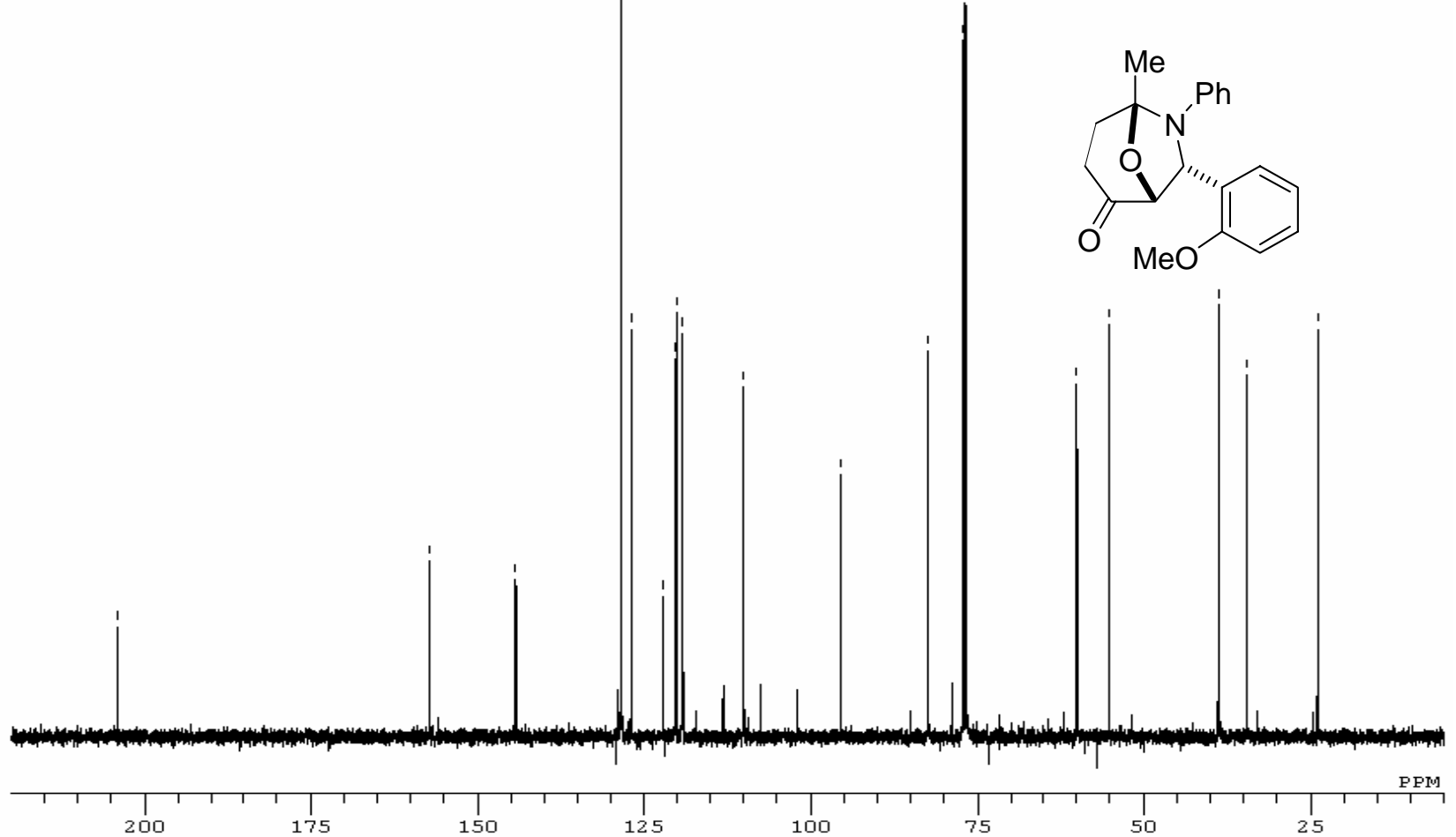


${ }^{1} \mathrm{H}$ NMR of Compound exo-11c

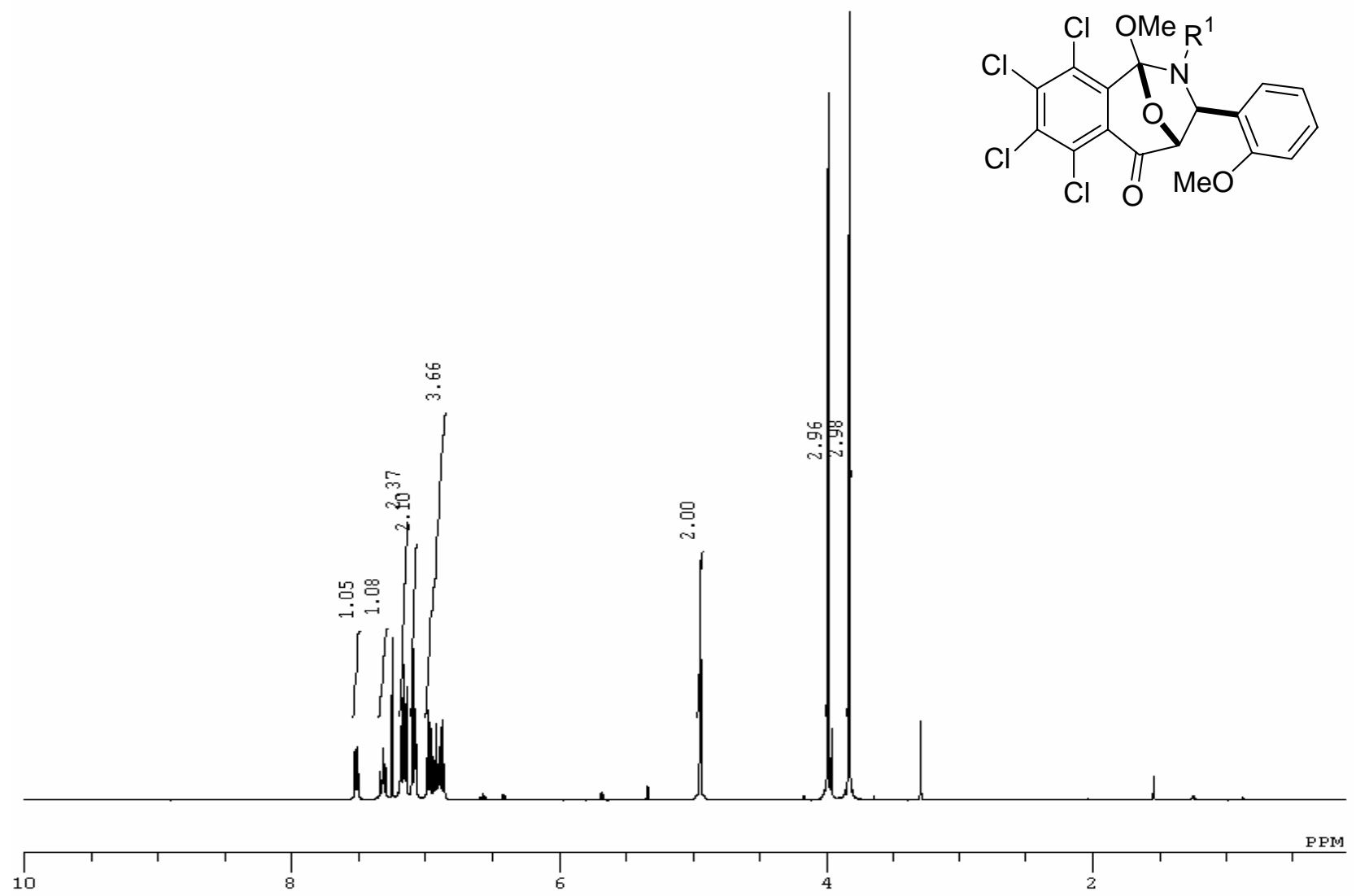

${ }^{13} \mathrm{C}$ NMR of Compound exo-11c

i

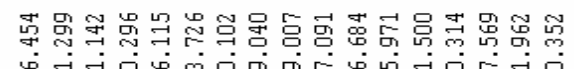

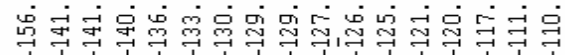

LLLLLLL

1)

品品总

11

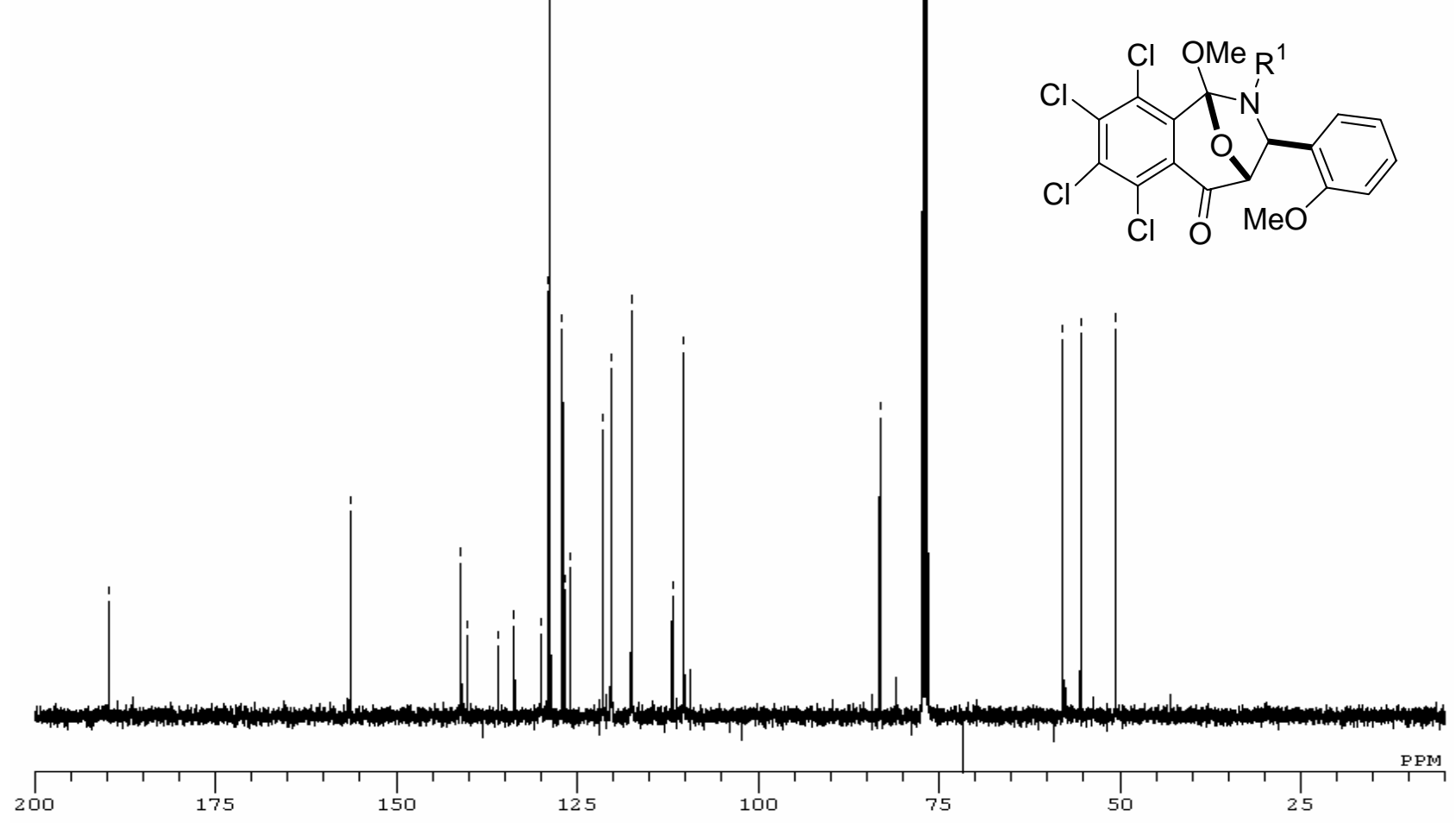




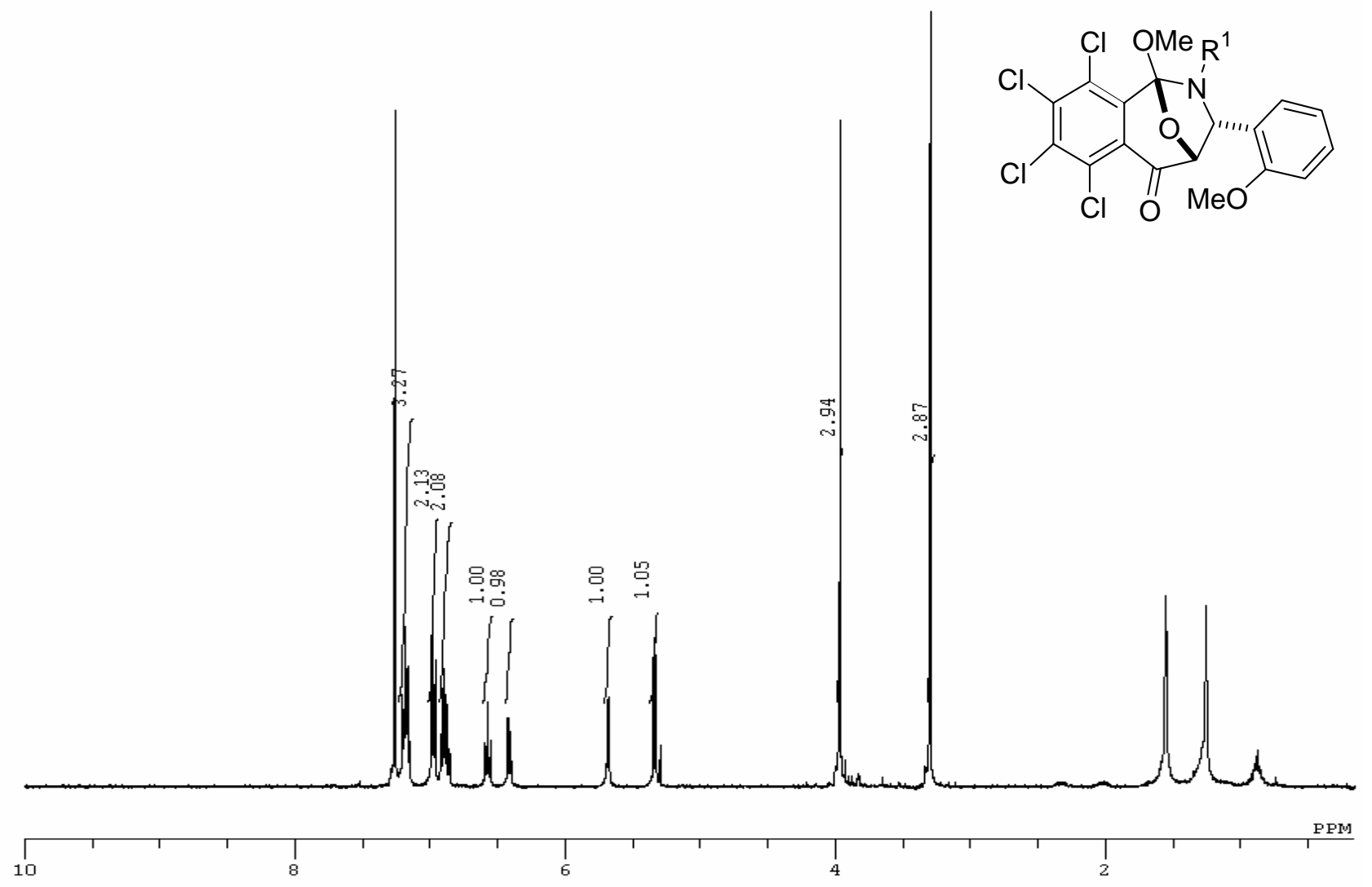

${ }^{13} \mathrm{C}$ NMR of Compound endo-11c

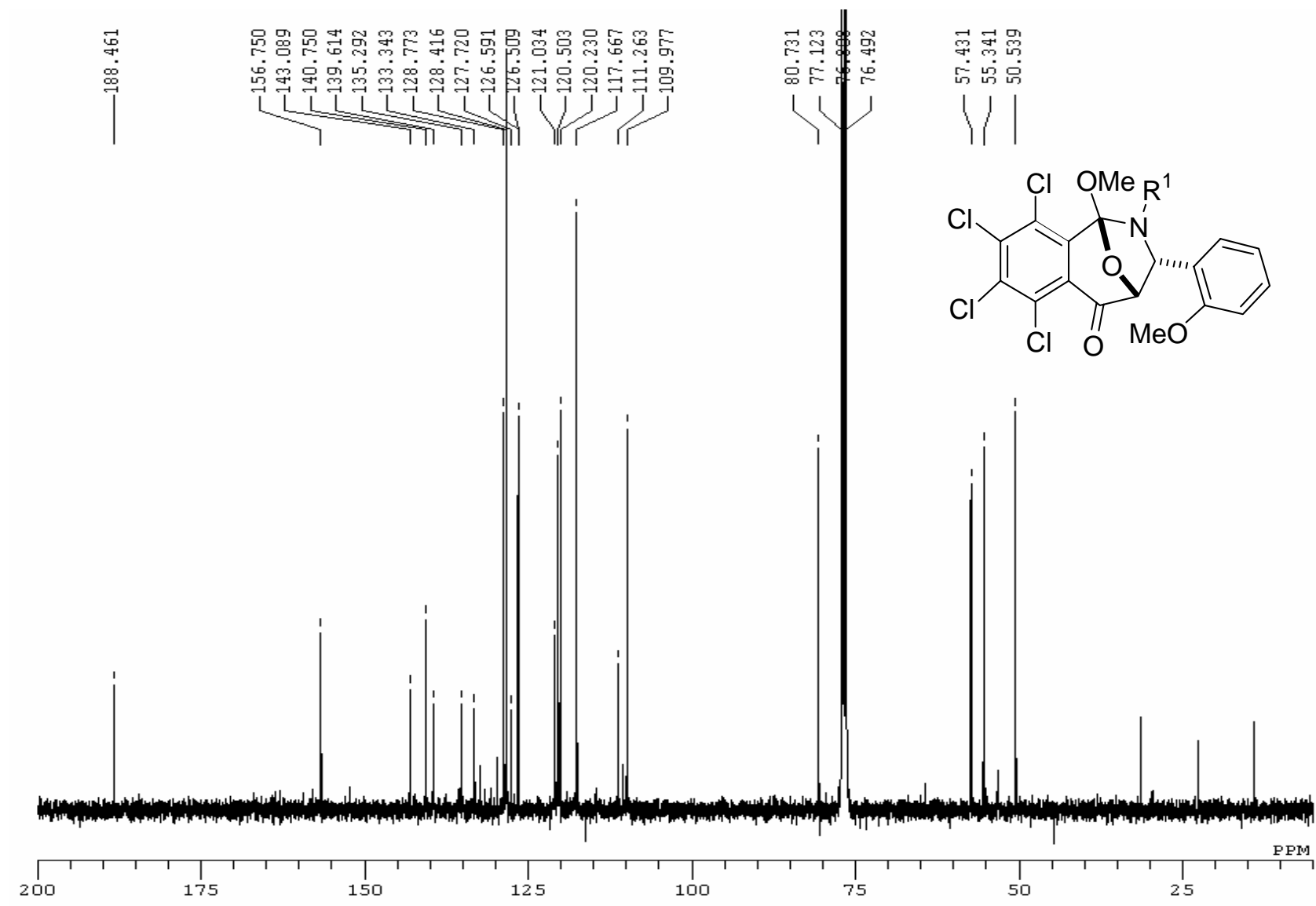




$$
\text { X-ray Structure Report of exo-3g }
$$

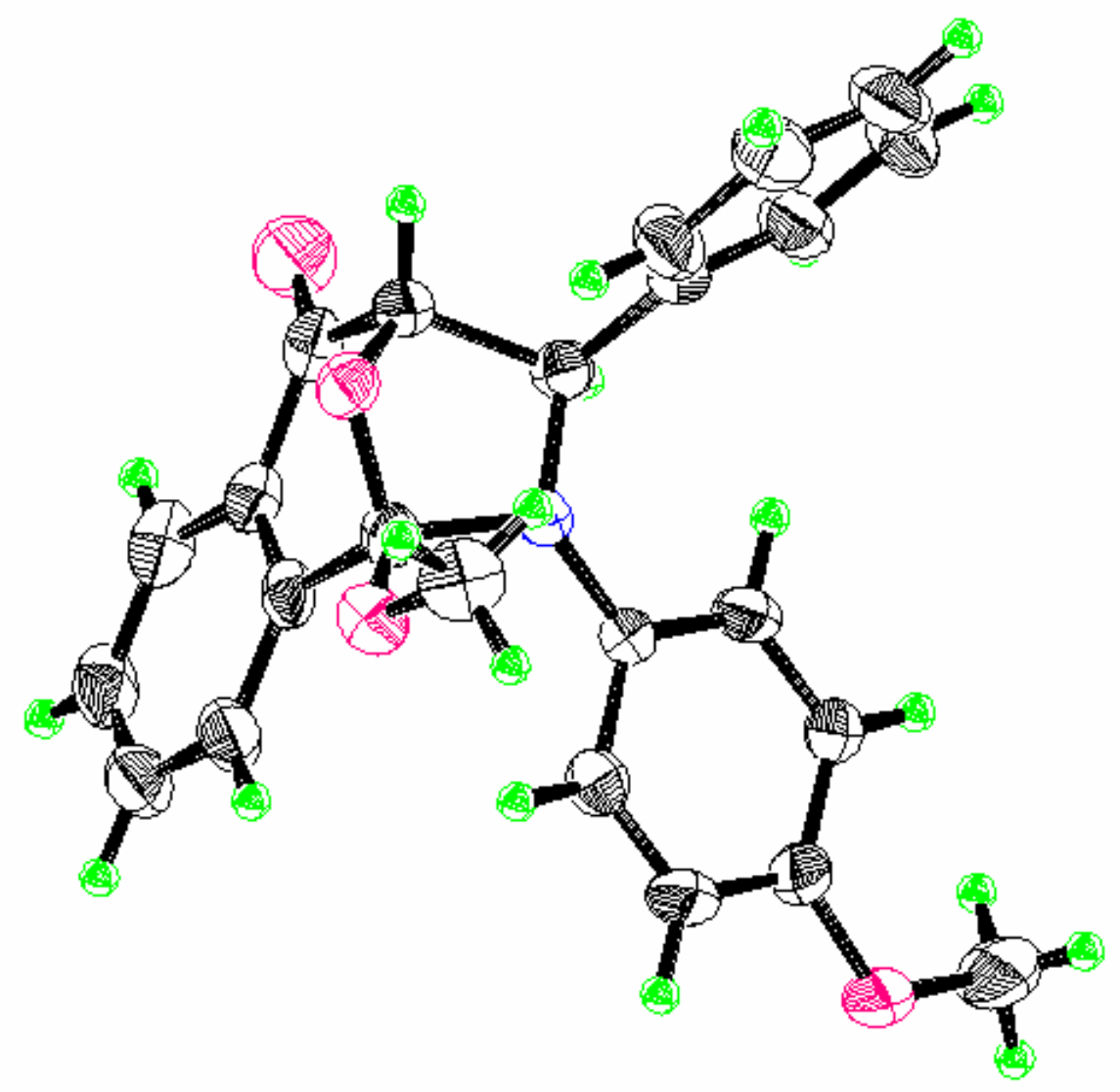

J anuary 27, 2004 


\section{Experimental}

\section{Data Collection}

A colorless prismatic crystal of $\mathrm{C}_{24} \mathrm{H}_{21} \mathrm{NO}_{4}$ having approximate dimensions of $0.32 \times 0.58 \times 1.00 \mathrm{~mm}$ was mounted on a glass fiber. All measurements were made on a Rigaku AFC5S diffractometer with graphite monochromated Mo-K $\alpha$ radiation.

Cell constants and an orientation matrix for data collection, obtained from a least-squares refinement using the setting angles of 20 carefully centered reflections in the range $20.02<2 \theta<27.65^{\circ}$ corresponded to a primitive monoclinic cell with dimensions:

$$
\begin{aligned}
& \mathrm{a}=8.476(9) \AA \\
& \mathrm{b}=12.54(1) \AA \quad \quad \quad \beta=95.71(8)^{\mathrm{O}} \\
& \mathrm{c}=18.38(1) \AA \\
& \mathrm{V}=1943(3) \AA^{3}
\end{aligned}
$$

For $Z=4$ and F.W. $=387.43$, the calculated density is $1.32 \mathrm{~g} / \mathrm{cm}^{3}$. The systematic absences of:

hOl: $h \pm 2 n$

Ok0: $k \pm 2 n$

uniquely determine the space group to be:

$$
\text { P21/a (\#14) }
$$

The data were collected at a temperature of $23 \pm 1^{\circ} \mathrm{C}$ using the $\omega-2 \theta$ scan technique to a maximum $2 \theta$ value of $55.0^{\circ}$. Omega scans of several intense reflections, made prior to data collection, had an average width at half-height of $0.08^{\circ}$ with a take-off angle of $6.0^{\circ}$. Scans of $(1.47+0.30 \tan \theta)^{0}$ were made at a speed of $16.0 \% / \mathrm{min}$ (in $\omega)$. The weak reflections $(I<10.0 \sigma(I))$ were rescanned (maximum of 3 scans) and the counts were accumulated to ensure good counting statistics. Stationary background counts were recorded on each side of the reflection. The ratio of peak counting time to background counting time was 2:1. The diameter of the incident beam collimator was $1.0 \mathrm{~mm}$, the crystal to detector distance was $258 \mathrm{~mm}$, and the detector aperture was 6.0 x $6.0 \mathrm{~mm}$ (horizontal x vertical). 


\section{Data Reduction}

Of the 4966 reflections which were collected, 4660 were unique $\left(R_{\text {int }}=0.045\right)$. The intensities of three representative reflections were measured after every 150 reflections. No decay correction was applied.

The linear absorption coefficient, $\mu$, for Mo-K $\alpha$ radiation is $0.9 \mathrm{~cm}^{-1}$. The data were corrected for Lorentz and polarization effects.

\section{$\underline{\text { Structure Solution and Refinement }}$}

The structure was solved by direct methods ${ }^{1}$ and expanded using Fourier techniques ${ }^{2}$. The non-hydrogen atoms were refined anisotropically. Hydrogen atoms were included but not refined. The final cycle of full-matrix least-squares refinement 3 on $\mathrm{F}$ was based on 1980 observed reflections $(\mathrm{I}>2.00 \sigma(\mathrm{I}))$ and 262 variable parameters and converged (largest parameter shift was 0.00 times its esd) with unweighted and weighted agreement factors of:

$$
\begin{gathered}
\mathrm{R}=\Sigma|| \mathrm{Fo}|-| \mathrm{FC}|| / \Sigma|\mathrm{Fo}|=0.067 \\
\mathrm{R}_{\mathrm{W}}=\left[\Sigma \mathrm{W}(|\mathrm{Fo}|-\mid \mathrm{FC})^{2} / \Sigma \mathrm{WFo}^{2}\right]^{1 / 2}=0.069
\end{gathered}
$$

The standard deviation of an observation of unit weight 4 was 2.05. The weighting scheme was based on counting statistics and included a factor $(p=0.018)$ to downweight the intense reflections. Plots of $\Sigma w(|F o|-|F c|)^{2}$ versus $|F o|$, reflection order in data collection, $\sin \theta / \lambda$ and various classes of indices showed no unusual trends. The maximum and minimum peaks on the final difference Fourier map corresponded to 0.60 and $-0.41 \mathrm{e}^{-} / \AA^{3}$, respectively.

Neutral atom scattering factors were taken from Cromer and Waber 5. Anomalous dispersion effects were included in $\mathrm{Fcal}^{6}{ }^{6}$; the values for $\Delta \mathrm{f}^{\prime}$ and $\Delta \mathrm{f}^{\prime \prime}$ were those of Creagh and McAuley7. The values for the mass attenuation coefficients are those of Creagh and Hubbell 8 . All calculations were performed using the teXsan 9 crystallographic software package of Molecular Structure Corporation. 


\section{References}

(1) SIR92: Altomare, A., Cascarano, M., Giacovazzo, C., Guagliardi, A. (1994). J . Appl. Cryst., 26, 343.

(2) DIRDIF 94: Beurskens, P.T., Admiraal, G., Beurskens, G., Bosman, W.P., de Gelder, R., I srael, R. and Smits, J.M.M.(1994). The DIRDIF-94 program system, Technical Report of the Crystallography Laboratory, University of Nijmegen, The Netherlands.

(3) Least Squares function minimized:

$$
\begin{aligned}
& \Sigma w\left(\left|F_{0}\right|-\left|F_{c}\right|\right)^{2} \text { where } \\
& w=1 /\left[\sigma^{2}(\mathrm{Fo})\right]=\left[\sigma^{2} \mathrm{c}(\mathrm{Fo})+\mathrm{p}^{2} \mathrm{Fo}^{2} / 4\right]^{-1} \\
& \sigma_{\mathrm{C}}(\mathrm{Fo})=\text { e.s.d. based on counting statistics } \\
& p=\text { p-factor }
\end{aligned}
$$

(4) Standard deviation of an observation of unit weight:

$$
\begin{aligned}
& {\left[\sum m\left(\mathrm{~F}_{\mathrm{o}}|-| \mathrm{F}_{\mathrm{C}}\right)^{2} /\left(\mathrm{N}_{\mathrm{O}}-\mathrm{N}_{\mathrm{v}}\right)\right]^{1 / 2}} \\
& \text { where: } \quad \begin{aligned}
\mathrm{N}_{\mathrm{O}} & =\text { number of observations } \\
\mathrm{N}_{\mathrm{V}} & =\text { number of variables }
\end{aligned}
\end{aligned}
$$

(5) Cromer, D. T. \& Waber, J . T.; "International Tables for X-ray Crystallography", Vol. IV, The Kynoch Press, Birmingham, England, Table 2.2 A (1974).

(6) I bers, J . A. \& Hamilton, W. C.; Acta Crystallogr., 17, 781 (1964).

(7) Creagh, D. C. \& McAuley, W.J ;; "International Tables for Crystallography", Vol C, (A.J .C. Wilson, ed.), Kluwer Academic Publishers, Boston, Table 4.2.6.8, pages 219-222 (1992). 
(8) Creagh, D. C. \& Hubbell, J .H..; "International Tables for Crystallography", Vol C, (A.J .C. Wilson, ed.), Kluwer Academic Publishers, Boston, Table 4.2.4.3, pages 200-206 (1992).

(9) teXsan for Windows version 1.06: Crystal Structure Analysis Package, Molecular Structure Corporation (1997-9). 
A. Crystal Data

Empirical Formula

Formula Weight

Crystal Color, Habit

Crystal Dimensions

Crystal System

Lattice Type

No. of Reflections Used for Unit

Cell Determination (2 $\theta$ range)

Omega Scan Peak Width

at Half-height

Lattice Parameters

Space Group

$Z$ value

$D_{\text {calc }}$

$F_{000}$

$\mu(\operatorname{MoK} \alpha)$
$\mathrm{C}_{24} \mathrm{H}_{21} \mathrm{NO}_{4}$

387.43

colorless, prismatic

$0.32 \times 0.58 \times 1.00 \mathrm{~mm}$

monoclinic

Primitive

$20\left(20.0-27.6^{0}\right)$

$0.08^{\circ}$

$a=8.476(9) \AA$

$\mathrm{b}=12.54(1) \AA$

$\mathrm{c}=18.38(1) \AA$

$\beta=95.71(8) \circ$

$V=1943(3) \AA^{3}$

P21/a (\#14)

4

$1.324 \mathrm{~g} / \mathrm{cm}^{3}$

816.00

$0.90 \mathrm{~cm}^{-1}$ 
B. Intensity M easurements

Diffractometer

Radiation

Attenuator

Take-off Angle

Detector Aperture

Crystal to Detector Distance

Voltage, Current

Temperature

Scan Type

Scan Rate

Scan Width

$2 \theta \max$

No. of Reflections Measured

Corrections
Rigaku AFC5S

$\operatorname{MoK} \alpha(\lambda=0.71069 \AA)$

graphite monochromated

Zr foil (factors $=1.00,2.26,5.12,11.58$ )

$6.0^{\circ}$

$6.0 \mathrm{~mm}$ horizontal

$6.0 \mathrm{~mm}$ vertical

$258 \mathrm{~mm}$

$50 \mathrm{kV}, 30 \mathrm{~mA}$

$23.0^{\circ} \mathrm{C}$

$\omega-2 \theta$

$16.0 \%$ min (in $\omega$ ) (up to 3 scans)

$(1.47+0.30 \tan \theta)^{\mathrm{O}}$

$55.0^{\circ}$

Total : 4966

Unique: $4660\left(R_{\text {int }}=0.045\right)$

Lorentz-polarization 
C. Structure Solution and Refinement

Structure Solution

Refinement

Function Minimized

Least Squares Weights

p-factor

Anomalous Dispersion

No. Observations $(I>2.00 \sigma(I))$

No. Variables

Reflection/Parameter Ratio

Residuals: R; Rw

Goodness of Fit Indicator

Max Shift/Error in Final Cycle

Maximum peak in Final Diff. Map

Minimum peak in Final Diff. Map
Direct Methods (SIR92)

Full-matrix least-squares on $\mathrm{F}$

$\Sigma w(|F o|-|F c|)^{2}$

$1 / \sigma^{2}(\mathrm{Fo})=4 \mathrm{Fo}^{2} / \sigma^{2}\left(\mathrm{Fo}^{2}\right)$

0.0180

All non-hydrogen atoms

1980

262

7.56

$0.067 ; 0.069$

2.05

0.00

$0.60 \mathrm{e}^{-} / \AA^{3}$

$-0.41 e^{-} / \AA^{3}$ 
Table 1. Atomic coordinates and $\mathrm{B}_{\mathrm{iso}} / \mathrm{B}_{\mathrm{eq}}$

$\begin{array}{lllll}\text { atom } & \mathrm{x} & \mathrm{y} & \mathrm{z} & \mathrm{B} \text { eq } \\ \mathrm{O}(1) & 0.4634(3) & -0.1006(2) & 0.1586(1) & 2.95(6) \\ \mathrm{O}(2) & 0.8294(3) & 0.0304(2) & 0.1819(1) & 4.94(8) \\ \mathrm{O}(3) & 0.1263(3) & 0.2583(2) & 0.4510(1) & 4.00(7) \\ \mathrm{O}(4) & 0.2868(3) & -0.1509(2) & 0.2397(1) & 3.14(6) \\ \mathrm{N}(1) & 0.3550(3) & 0.0320(2) & 0.2199(1) & 2.62(6) \\ \mathrm{C}(1) & 0.5426(4) & -0.0965(2) & 0.2862(2) & 2.70(8) \\ \mathrm{C}(2) & 0.5286(4) & -0.1464(3) & 0.3530(2) & 3.49(9) \\ \mathrm{C}(3) & 0.6595(5) & -0.1572(3) & 0.4037(2) & 4.0(1) \\ \mathrm{C}(4) & 0.8058(5) & -0.1192(3) & 0.3888(2) & 4.3(1) \\ \mathrm{C}(5) & 0.8227(4) & -0.0703(3) & 0.3218(2) & 3.9(1) \\ \mathrm{C}(6) & 0.6911(4) & -0.0597(3) & 0.2708(2) & 2.85(8) \\ \mathrm{C}(7) & 0.7075(4) & -0.0089(3) & 0.1979(2) & 3.25(9) \\ \mathrm{C}(8) & 0.5559(4) & -0.0079(3) & 0.1468(2) & 3.00(8) \\ \mathrm{C}(9) & 0.4488(4) & 0.0864(2) & 0.1674(2) & 2.59(8) \\ \mathrm{C}(10) & 0.4054(4) & -0.0802(2) & 0.2280(2) & 2.74(8) \\ \mathrm{C}(11) & 0.3494(4) & 0.1336(3) & 0.1028(2) & 2.77(8) \\ \mathrm{C}(12) & 0.3596(4) & 0.2413(3) & 0.0878(2) & 3.8(1) \\ \mathrm{C}(13) & 0.2705(5) & 0.2841(3) & 0.0274(3) & 5.0(1) \\ \mathrm{C}(14) & 0.1759(5) & 0.2211(4) & -0.0193(2) & 5.0(1) \\ \mathrm{C}(15) & 0.1661(5) & 0.1132(3) & -0.0051(2) & 4.7(1) \\ \mathrm{C}(16) & 0.2516(5) & 0.0703(3) & 0.0561(2) & 4.0(1) \\ \mathrm{C}(17) & 0.3014(4) & 0.0907(3) & 0.2783(2) & 2.42(8) \\ \mathrm{C}(18) & 0.3075(4) & 0.2010(3) & 0.2799(2) & 3.34(9) \\ \mathrm{C}(19) & 0.2506(4) & 0.2603(3) & 0.3360(2) & 3.35(9) \\ \mathrm{C}(20) & 0.1858(4) & 0.2076(3) & 0.3925(2) & 3.03(8) \\ \mathrm{C}(21) & 0.1773(4) & 0.0979(3) & 0.3913(2) & 3.33(9) \\ \mathrm{C}(22) & 0.2332(4) & 0.0396(3) & 0.3357(2) & 3.01(8) \\ \mathrm{C}(23) & 0.1373(5) & 0.3713(3) & 0.4527(2) & 5.5(1) \\ \mathrm{C}(24) & 0.1459(4) & -0.1460(3) & 0.1896(2) & 4.2(1) \\ \mathrm{H}(1) & 0.4286 & -0.1732 & 0.3637 & 4.187 \\ \mathrm{H}(2) & 0.6487 & -0.1910 & 0.4492 & 4.754 \\ \mathrm{H}(3) & 0.8949 & -0.1264 & 0.4241 & 5.109 \\ \mathrm{H}(4) & 0.9233 & -0.0446 & 0.3112 & 4.696 \\ \mathrm{H}(5) & 0.5779 & -0.0031 & 0.0972 & 3.600 \\ \mathrm{H}(6) & 0.5125 & 0.1404 & 0.1918 & 3.103 \\ \mathrm{H}(7) & 0.4275 & 0.2858 & 0.1188 & 4.585 \\ \mathrm{H}(8) & 0.2753 & 0.3586 & 0.0184 & 6.058\end{array}$




$\begin{array}{llrrr}H(9) & 0.1175 & 0.2512 & -0.0612 & 5.940 \\ H(10) & 0.1008 & 0.0685 & -0.0371 & 5.614 \\ H(11) & 0.2431 & -0.0037 & 0.0661 & 4.808\end{array}$


Table 1. Atomic coordinates and $\mathrm{B}_{\mathrm{iso}} / \mathrm{B}_{\mathrm{eq}}$ (continued)

$\begin{array}{lcccl}\text { atom } & x & y & z & B_{\text {eq }} \\ \mathrm{H}(12) & 0.3520 & 0.2376 & 0.2416 & 4.009 \\ \mathrm{H}(13) & 0.2561 & 0.3360 & 0.3355 & 4.015 \\ \mathrm{H}(14) & 0.1320 & 0.0617 & 0.4296 & 3.999 \\ \mathrm{H}(15) & 0.2257 & -0.0360 & 0.3362 & 3.609 \\ \mathrm{H}(16) & 0.1414 & 0.3953 & 0.5019 & 6.619 \\ \mathrm{H}(17) & 0.2306 & 0.3931 & 0.4322 & 6.619 \\ \mathrm{H}(18) & 0.0471 & 0.4011 & 0.4252 & 6.619 \\ \mathrm{H}(19) & 0.0557 & -0.1415 & 0.2162 & 4.984 \\ \mathrm{H}(20) & 0.1501 & -0.0848 & 0.1593 & 4.984 \\ \mathrm{H}(21) & 0.1387 & -0.2083 & 0.1600 & 4.984\end{array}$

$B_{\text {eq }}=8 / 3 \pi^{2}\left(U_{11}\left(a a^{*}\right)^{2}+U_{22}\left(b b^{*}\right)^{2}+U_{33}\left(c c^{*}\right)^{2}+2 U_{12}\left(a a^{*} b b^{*}\right) \cos \gamma+2 U_{13}\left(a a^{*} c c^{*}\right) \cos \beta+2 U_{23}\left(b b^{*} c c^{*}\right) \cos \alpha\right)$ 
Table 2. Anisotropic Displacement Parameters

$\begin{array}{lllllll}\text { atom } & U_{11} & U_{22} & U_{33} & U_{12} & U_{13} & U_{23} \\ \mathrm{O}(1) & 0.047(2) & 0.032(1) & 0.033(1) & 0.004(1) & 0.004(1) & -0.007(1) \\ \mathrm{O}(2) & 0.040(2) & 0.076(2) & 0.073(2) & 0.001(1) & 0.013(1) & 0.016(2) \\ \mathrm{O}(3) & 0.073(2) & 0.048(2) & 0.034(1) & 0.013(1) & 0.019(1) & 0.000(1) \\ \mathrm{O}(4) & 0.045(1) & 0.035(1) & 0.039(1) & -0.006(1) & -0.002(1) & -0.000(1) \\ \mathrm{N}(1) & 0.044(2) & 0.026(2) & 0.031(2) & 0.003(1) & 0.008(1) & 0.003(1) \\ \mathrm{C}(1) & 0.042(2) & 0.024(2) & 0.036(2) & 0.006(2) & -0.002(2) & -0.005(2) \\ \mathrm{C}(2) & 0.052(2) & 0.036(2) & 0.043(2) & 0.006(2) & 0.001(2) & 0.005(2) \\ \mathrm{C}(3) & 0.066(3) & 0.045(2) & 0.038(2) & 0.012(2) & -0.000(2) & 0.005(2) \\ \mathrm{C}(4) & 0.061(3) & 0.059(3) & 0.038(2) & 0.024(2) & -0.017(2) & -0.006(2) \\ \mathrm{C}(5) & 0.043(2) & 0.045(2) & 0.060(3) & 0.006(2) & -0.002(2) & -0.010(2) \\ \mathrm{C}(6) & 0.035(2) & 0.034(2) & 0.040(2) & 0.005(2) & 0.003(2) & -0.006(2) \\ \mathrm{C}(7) & 0.040(2) & 0.036(2) & 0.049(2) & 0.009(2) & 0.007(2) & -0.003(2) \\ \mathrm{C}(8) & 0.049(2) & 0.034(2) & 0.032(2) & 0.006(2) & 0.011(2) & 0.002(2) \\ \mathrm{C}(9) & 0.037(2) & 0.029(2) & 0.033(2) & -0.003(2) & 0.007(2) & -0.001(2) \\ \mathrm{C}(10) & 0.044(2) & 0.025(2) & 0.036(2) & 0.001(2) & 0.004(2) & -0.003(2) \\ \mathrm{C}(11) & 0.040(2) & 0.039(2) & 0.028(2) & 0.006(2) & 0.012(2) & 0.006(2) \\ \mathrm{C}(12) & 0.060(3) & 0.043(2) & 0.043(2) & -0.001(2) & 0.008(2) & 0.010(2) \\ \mathrm{C}(13) & 0.073(3) & 0.051(3) & 0.070(3) & 0.010(2) & 0.018(3) & 0.030(2) \\ \mathrm{C}(14) & 0.057(3) & 0.085(4) & 0.046(3) & 0.013(3) & 0.009(2) & 0.030(3) \\ \mathrm{C}(15) & 0.066(3) & 0.071(3) & 0.039(2) & 0.010(2) & -0.003(2) & 0.003(2) \\ \mathrm{C}(16) & 0.067(3) & 0.047(2) & 0.037(2) & 0.008(2) & -0.004(2) & 0.004(2) \\ \mathrm{C}(17) & 0.033(2) & 0.030(2) & 0.029(2) & 0.003(2) & 0.003(1) & 0.003(2) \\ \mathrm{C}(18) & 0.060(3) & 0.036(2) & 0.034(2) & 0.002(2) & 0.016(2) & 0.007(2) \\ \mathrm{C}(19) & 0.059(2) & 0.031(2) & 0.039(2) & 0.012(2) & 0.014(2) & 0.003(2) \\ \mathrm{C}(20) & 0.042(2) & 0.041(2) & 0.031(2) & 0.008(2) & 0.004(2) & -0.001(2) \\ \mathrm{C}(21) & 0.047(2) & 0.044(2) & 0.037(2) & -0.003(2) & 0.015(2) & 0.007(2) \\ \mathrm{C}(22) & 0.044(2) & 0.027(2) & 0.044(2) & -0.003(2) & 0.008(2) & 0.002(2) \\ \mathrm{C}(23) & 0.108(4) & 0.056(3) & 0.050(3) & 0.013(3) & 0.030(2) & -0.012(2) \\ \mathrm{C}(24) & 0.044(2) & 0.057(3) & 0.055(3) & -0.013(2) & -0.001(2) & -0.007(2)\end{array}$

The general temperature factor expression:exp $\left(-2 \pi^{2}\left(a * 2 U_{11} h^{2}+b * 2 U_{22} k^{2}+\left.c^{2} U_{33}\right|^{2}\right.\right.$ $\left.\left.+2 a * b * U_{12} h k+2 a * c * U_{13} h l+2 b * c * U_{23} k l\right)\right)$ 
Table 3. Bond Lengths $(\AA)$

$\begin{array}{llllll}\text { atom } & \text { atom } & \text { distance } & \text { atom } & \text { atom } & \text { distance } \\ \mathrm{O}(1) & \mathrm{C}(8) & 1.431(5) & \mathrm{O}(1) & \mathrm{C}(10) & 1.435(5) \\ \mathrm{O}(2) & \mathrm{C}(7) & 1.208(5) & \mathrm{O}(3) & \mathrm{C}(20) & 1.387(5) \\ \mathrm{O}(3) & \mathrm{C}(23) & 1.419(6) & \mathrm{O}(4) & \mathrm{C}(10) & 1.373(5) \\ \mathrm{O}(4) & \mathrm{C}(24) & 1.435(6) & \mathrm{N}(1) & \mathrm{C}(9) & 1.476(6) \\ \mathrm{N}(1) & \mathrm{C}(10) & 1.474(5) & \mathrm{N}(1) & \mathrm{C}(17) & 1.412(5) \\ \mathrm{C}(1) & \mathrm{C}(2) & 1.392(6) & \mathrm{C}(1) & \mathrm{C}(6) & 1.396(6) \\ \mathrm{C}(1) & \mathrm{C}(10) & 1.514(6) & \mathrm{C}(2) & \mathrm{C}(3) & 1.384(7) \\ \mathrm{C}(2) & \mathrm{H}(1) & 0.95 & \mathrm{C}(3) & \mathrm{C}(4) & 1.382(7) \\ \mathrm{C}(3) & \mathrm{H}(2) & 0.95 & \mathrm{C}(4) & \mathrm{C}(5) & 1.395(7) \\ \mathrm{C}(4) & \mathrm{H}(3) & 0.95 & \mathrm{C}(5) & \mathrm{C}(6) & 1.391(7) \\ \mathrm{C}(5) & \mathrm{H}(4) & 0.95 & \mathrm{C}(6) & \mathrm{C}(7) & 1.501(7) \\ \mathrm{C}(7) & \mathrm{C}(8) & 1.515(7) & \mathrm{C}(8) & \mathrm{C}(9) & 1.560(6) \\ \mathrm{C}(8) & \mathrm{H}(5) & 0.95 & \mathrm{C}(9) & \mathrm{C}(11) & 1.507(6) \\ \mathrm{C}(9) & \mathrm{H}(6) & 0.95 & \mathrm{C}(11) & \mathrm{C}(12) & 1.382(7) \\ \mathrm{C}(11) & \mathrm{C}(16) & 1.383(7) & \mathrm{C}(12) & \mathrm{C}(13) & 1.387(7) \\ \mathrm{C}(12) & \mathrm{H}(7) & 0.95 & \mathrm{C}(13) & \mathrm{C}(14) & 1.367(8) \\ \mathrm{C}(13) & \mathrm{H}(8) & 0.95 & \mathrm{C}(14) & \mathrm{C}(15) & 1.383(8) \\ \mathrm{C}(14) & \mathrm{H}(9) & 0.95 & \mathrm{C}(15) & \mathrm{C}(16) & 1.384(7) \\ \mathrm{C}(15) & \mathrm{H}(10) & 0.95 & \mathrm{C}(16) & \mathrm{H}(11) & 0.95 \\ \mathrm{C}(17) & \mathrm{C}(18) & 1.383(6) & \mathrm{C}(17) & \mathrm{C}(22) & 1.408(6) \\ \mathrm{C}(18) & \mathrm{C}(19) & 1.395(6) & \mathrm{C}(18) & \mathrm{H}(12) & 0.95 \\ \mathrm{C}(19) & \mathrm{C}(20) & 1.389(6) & \mathrm{C}(19) & \mathrm{H}(13) & 0.95 \\ \mathrm{C}(20) & \mathrm{C}(21) & 1.378(7) & \mathrm{C}(21) & \mathrm{C}(22) & 1.378(7) \\ \mathrm{C}(21) & \mathrm{H}(14) & 0.95 & \mathrm{C}(22) & \mathrm{H}(15) & 0.95 \\ \mathrm{C}(23) & \mathrm{H}(16) & 0.95 & \mathrm{C}(23) & \mathrm{H}(17) & 0.95 \\ \mathrm{C}(23) & \mathrm{H}(18) & 0.95 & \mathrm{C}(24) & \mathrm{H}(19) & 0.95 \\ \mathrm{C}(24) & \mathrm{H}(20) & 0.95 & \mathrm{C}(24) & \mathrm{H}(21) & 0.95\end{array}$


Table 4. Bond Angles( $\left.{ }^{\circ}\right)$

\begin{tabular}{|c|c|c|c|c|c|c|c|}
\hline atom & atom & atom & angle & atom & atom & atom & angle \\
\hline$C(8)$ & $\mathrm{O}(1)$ & $C(10)$ & $103.3(3)$ & $C(20)$ & $\mathrm{O}(3)$ & $C(23)$ & $116.5(4)$ \\
\hline$C(10)$ & $\mathrm{O}(4)$ & $C(24)$ & $116.5(4)$ & $C(9)$ & $N(1)$ & $C(10)$ & 109.7(4) \\
\hline$C(9)$ & $N(1)$ & $C(17)$ & 119.4(4) & $C(10)$ & $N(1)$ & $C(17)$ & $122.2(4)$ \\
\hline$C(2)$ & $C(1)$ & $C(6)$ & $119.1(5)$ & $C(2)$ & $C(1)$ & $C(10)$ & 123.8(5) \\
\hline$C(6)$ & $C(1)$ & $C(10)$ & $117.1(4)$ & $C(1)$ & $C(2)$ & $C(3)$ & $120.3(5)$ \\
\hline$C(1)$ & $C(2)$ & $H(1)$ & 119 & $C(3)$ & $C(2)$ & $H(1)$ & 119 \\
\hline$C(2)$ & $C(3)$ & $C(4)$ & $120.5(5)$ & $C(2)$ & $C(3)$ & $H(2)$ & 119 \\
\hline$C(4)$ & $C(3)$ & $H(2)$ & 119 & $C(3)$ & $C(4)$ & $C(5)$ & $120.0(5)$ \\
\hline$C(3)$ & $C(4)$ & $\mathrm{H}(3)$ & 120 & $C(5)$ & $C(4)$ & $\mathrm{H}(3)$ & 120 \\
\hline$C(4)$ & $C(5)$ & $C(6)$ & 119.4(5) & $C(4)$ & C(5) & $\mathrm{H}(4)$ & 120 \\
\hline$C(6)$ & $C(5)$ & $\mathrm{H}(4)$ & 120 & $C(1)$ & $C(6)$ & $C(5)$ & $120.6(5)$ \\
\hline$C(1)$ & $C(6)$ & $C(7)$ & 119.2(4) & $C(5)$ & $C(6)$ & $C(7)$ & $120.2(5)$ \\
\hline $\mathrm{O}(2)$ & $C(7)$ & $C(6)$ & $123.2(5)$ & $\mathrm{O}(2)$ & $C(7)$ & $C(8)$ & $122.6(5)$ \\
\hline$C(6)$ & $C(7)$ & $C(8)$ & $114.1(4)$ & $\mathrm{O}(1)$ & $C(8)$ & $C(7)$ & $110.0(4)$ \\
\hline $\mathrm{O}(1)$ & $C(8)$ & $C(9)$ & $103.8(4)$ & $\mathrm{O}(1)$ & $C(8)$ & $H(5)$ & 111 \\
\hline$C(7)$ & $C(8)$ & $C(9)$ & 109.5(4) & $C(7)$ & $C(8)$ & $H(5)$ & 111 \\
\hline$C(9)$ & $C(8)$ & $H(5)$ & 111 & $N(1)$ & $C(9)$ & $C(8)$ & $100.0(4)$ \\
\hline$N(1)$ & $C(9)$ & $C(11)$ & $113.6(4)$ & $N(1)$ & $C(9)$ & $H(6)$ & 109 \\
\hline$C(8)$ & $C(9)$ & $C(11)$ & $113.5(4)$ & $C(8)$ & $C(9)$ & $H(6)$ & 109 \\
\hline$C(11)$ & $C(9)$ & $H(6)$ & 109 & $\mathrm{O}(1)$ & $C(10)$ & $\mathrm{O}(4)$ & $110.2(4)$ \\
\hline$O(1)$ & $C(10)$ & $N(1)$ & 101.7(3) & $\mathrm{O}(1)$ & $C(10)$ & $C(1)$ & $107.3(4)$ \\
\hline O(4) & $C(10)$ & $N(1)$ & $115.0(4)$ & $\mathrm{O}(4)$ & $C(10)$ & $C(1)$ & $108.9(4)$ \\
\hline$N(1)$ & $C(10)$ & $C(1)$ & $113.2(4)$ & $C(9)$ & $C(11)$ & $C(12)$ & $120.0(5)$ \\
\hline$C(9)$ & $C(11)$ & $C(16)$ & $121.1(4)$ & $C(12)$ & $C(11)$ & $C(16)$ & $118.8(5)$ \\
\hline$C(11)$ & $C(12)$ & $C(13)$ & $119.9(5)$ & $C(11)$ & $C(12)$ & $\mathrm{H}(7)$ & 120 \\
\hline$C(13)$ & $C(12)$ & $H(7)$ & 120 & $C(12)$ & $C(13)$ & $C(14)$ & $121.2(5)$ \\
\hline$C(12)$ & $C(13)$ & $\mathrm{H}(8)$ & 119 & $C(14)$ & $C(13)$ & $\mathrm{H}(8)$ & 119 \\
\hline$C(13)$ & $C(14)$ & $C(15)$ & 119.3(5) & $C(13)$ & $C(14)$ & $H(9)$ & 120 \\
\hline$C(15)$ & $C(14)$ & $H(9)$ & 120 & $C(14)$ & $C(15)$ & $C(16)$ & $119.8(6)$ \\
\hline$C(14)$ & $C(15)$ & $\mathrm{H}(10)$ & 120 & $C(16)$ & $C(15)$ & $H(10)$ & 120 \\
\hline$C(11)$ & $C(16)$ & $C(15)$ & $121.0(5)$ & $C(11)$ & $C(16)$ & $\mathrm{H}(11)$ & 119 \\
\hline$C(15)$ & $C(16)$ & $\mathrm{H}(11)$ & 119 & $N(1)$ & $C(17)$ & $C(18)$ & $121.6(4)$ \\
\hline$N(1)$ & $C(17)$ & $C(22)$ & $121.3(4)$ & $C(18)$ & $C(17)$ & $C(22)$ & $117.1(4)$ \\
\hline$C(17)$ & $C(18)$ & $C(19)$ & $122.3(5)$ & $C(17)$ & $C(18)$ & $\mathrm{H}(12)$ & 118 \\
\hline$C(19)$ & $C(18)$ & $\mathrm{H}(12)$ & 118 & $C(18)$ & $C(19)$ & $C(20)$ & $119.3(5)$ \\
\hline$C(18)$ & $C(19)$ & $\mathrm{H}(13)$ & 120 & $C(20)$ & $C(19)$ & $\mathrm{H}(13)$ & 120 \\
\hline O(3) & $C(20)$ & $C(19)$ & $124.3(4)$ & $\mathrm{O}(3)$ & $C(20)$ & $C(21)$ & $116.5(5)$ \\
\hline
\end{tabular}


$\mathrm{C}(19) \quad \mathrm{C}(20) \quad \mathrm{C}(21) \quad 119.2(5) \quad \mathrm{C}(20) \quad \mathrm{C}(21) \quad \mathrm{C}(22) \quad 121.3(5)$

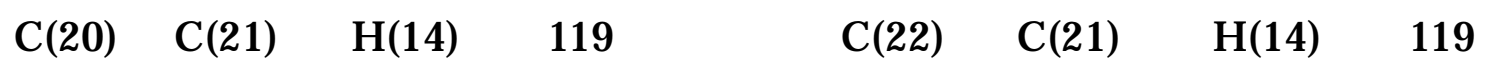

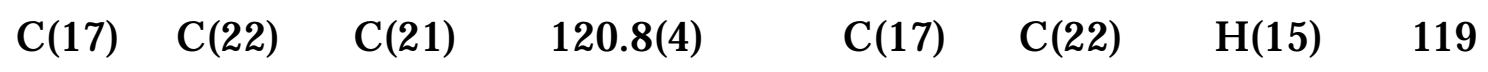


Table 4. Bond Angles( ${ }^{\circ}$ (continued)

$\begin{array}{llllllll}\text { atom } & \text { atom } & \text { atom } & \text { angle } & \text { atom } & \text { atom } & \text { atom } & \text { angle } \\ \mathrm{C}(21) & \mathrm{C}(22) & \mathrm{H}(15) & 119 & \mathrm{O}(3) & \mathrm{C}(23) & \mathrm{H}(16) & 109 \\ \mathrm{O}(3) & \mathrm{C}(23) & \mathrm{H}(17) & 109 & \mathrm{O}(3) & \mathrm{C}(23) & \mathrm{H}(18) & 109 \\ \mathrm{H}(16) & \mathrm{C}(23) & \mathrm{H}(17) & 109 & \mathrm{H}(16) & \mathrm{C}(23) & \mathrm{H}(18) & 109 \\ \mathrm{H}(17) & \mathrm{C}(23) & \mathrm{H}(18) & 109 & \mathrm{O}(4) & \mathrm{C}(24) & \mathrm{H}(19) & 109 \\ \mathrm{O}(4) & \mathrm{C}(24) & \mathrm{H}(20) & 109 & \mathrm{O}(4) & \mathrm{C}(24) & \mathrm{H}(21) & 109 \\ \mathrm{H}(19) & \mathrm{C}(24) & \mathrm{H}(20) & 109 & \mathrm{H}(19) & \mathrm{C}(24) & \mathrm{H}(21) & 109 \\ \mathrm{H}(20) & \mathrm{C}(24) & \mathrm{H}(21) & 109 & & & & \end{array}$


Table 5. Torsion Angles( $\left({ }^{\circ}\right)$

atom atom atom atom angle

$O(1) \quad C(8) \quad C(7) \quad O(2) \quad 149.1(5)$

$\mathrm{O}(1) \quad \mathrm{C}(8) \quad \mathrm{C}(9) \quad \mathrm{N}(1) \quad 26.8(4)$

$\mathrm{O}(1) \quad \mathrm{C}(10) \quad \mathrm{O}(4) \quad \mathrm{C}(24) \quad-63.1(5)$

$\mathrm{O}(1) \quad \mathrm{C}(10) \quad \mathrm{N}(1) \quad \mathrm{C}(17) \quad-174.3(4)$

$\mathrm{O}(1) \quad \mathrm{C}(10) \quad \mathrm{C}(1) \quad \mathrm{C}(6) \quad 39.8(5)$

$\mathrm{O}(2) \quad \mathrm{C}(7) \quad \mathrm{C}(6) \quad \mathrm{C}(5) \quad-4.9(8)$

$\mathrm{O}(3) \quad \mathrm{C}(20) \quad \mathrm{C}(19) \quad \mathrm{C}(18) \quad 179.8(4)$

$\mathrm{O}(4) \quad \mathrm{C}(10) \quad \mathrm{O}(1) \quad \mathrm{C}(8) \quad 166.9(3)$

$\mathrm{O}(4) \quad \mathrm{C}(10) \quad \mathrm{N}(1) \quad \mathrm{C}(17) \quad 66.7(6)$

$\mathrm{O}(4) \quad \mathrm{C}(10) \quad \mathrm{C}(1) \quad \mathrm{C}(6) \quad 159.0(4)$

$\mathrm{N}(1) \quad \mathrm{C}(9) \quad \mathrm{C}(11) \quad \mathrm{C}(12) \quad 122.7(5)$

$\mathrm{N}(1) \quad \mathrm{C}(10) \quad \mathrm{O}(1) \quad \mathrm{C}(8) \quad 44.4(4)$

$\mathrm{N}(1) \quad \mathrm{C}(10) \quad \mathrm{C}(1) \quad \mathrm{C}(2) \quad 109.0(5)$

$\mathrm{N}(1) \quad \mathrm{C}(17) \quad \mathrm{C}(18) \quad \mathrm{C}(19) \quad 178.0(5)$

$C(1) \quad C(2) \quad C(3) \quad C(4) \quad-0.3(8)$

$C(1) \quad C(6) \quad C(7) \quad C(8) \quad-2.4(6)$

$\mathrm{C}(1) \quad \mathrm{C}(10) \mathrm{O}(4) \quad \mathrm{C}(24) \quad 179.4(4)$

C(1) $\quad \mathrm{C}(10) \quad \mathrm{N}(1) \quad \mathrm{C}(17) \quad-59.5(6)$

$C(2) \quad C(1) \quad C(6) \quad C(7) \quad 178.1(4)$

$\mathrm{C}(3) \quad \mathrm{C}(2) \quad \mathrm{C}(1) \quad \mathrm{C}(6) \quad 1.3(7)$

$C(3) \quad C(4) \quad C(5) \quad C(6) \quad 0.5(8)$

$C(5) \quad C(6) \quad C(1) \quad C(10) \quad 179.1(4)$

$C(6) \quad C(7) \quad C(8) \quad C(9) \quad 80.6(5)$

$\begin{array}{lllll}C(7) & C(8) & O(1) & C(10) & 71.9(4)\end{array}$

$\mathrm{C}(8) \quad \mathrm{C}(9) \quad \mathrm{N}(1) \quad \mathrm{C}(10) \quad 0.4(5)$

$C(8) \quad C(9) \quad C(11) \quad C(12)-123.8(5)$

$\mathrm{C}(9) \quad \mathrm{N}(1) \quad \mathrm{C}(17) \quad \mathrm{C}(18) \quad 12.6(7)$

$\mathrm{C}(9) \quad \mathrm{C}(8) \quad \mathrm{O}(1) \quad \mathrm{C}(10) \quad-45.2(4)$

$C(9) \quad C(11) \quad C(16) \quad C(15)-177.0(5)$

$\mathrm{C}(10) \quad \mathrm{N}(1) \quad \mathrm{C}(17) \quad \mathrm{C}(18) \quad 156.9(4)$

C(11) $\quad \mathrm{C}(9) \quad \mathrm{N}(1) \quad \mathrm{C}(17) \quad-90.0(5)$

C(11) C(16) C(15) C(14) -1.1(9)

$C(12) \quad C(13) \quad C(14) \quad C(15) \quad 2(1)$

$C(13) \quad C(14) \quad C(15) \quad C(16) \quad 0(1)$

C(17) $\quad$ C(22) $\quad C(21) \quad C(20) \quad 0.1(8)$

$C(18) \quad C(19) \quad C(20) \quad C(21) \quad-0.9(8)$

$\mathrm{C}(19) \quad \mathrm{C}(20) \mathrm{O}(3) \quad \mathrm{C}(23) \quad-1.5(7)$ atom atom atom atom angle

$\mathrm{O}(1) \quad \mathrm{C}(8) \quad \mathrm{C}(7) \quad \mathrm{C}(6) \quad-32.9(5)$

$\mathrm{O}(1) \quad \mathrm{C}(8) \quad \mathrm{C}(9) \quad \mathrm{C}(11) \quad-94.7(4)$

$\mathrm{O}(1) \quad \mathrm{C}(10) \quad \mathrm{N}(1) \quad \mathrm{C}(9) \quad-27.0(5)$

$\mathrm{O}(1) \quad \mathrm{C}(10) \quad \mathrm{C}(1) \quad \mathrm{C}(2) \quad-139.6(4)$

$\mathrm{O}(2) \quad \mathrm{C}(7) \quad \mathrm{C}(6) \quad \mathrm{C}(1) \quad 175.6(5)$

$\mathrm{O}(2) \quad \mathrm{C}(7) \quad \mathrm{C}(8) \quad \mathrm{C}(9) \quad-97.4(6)$

$\mathrm{O}(3) \quad \mathrm{C}(20) \quad \mathrm{C}(21) \quad \mathrm{C}(22) \quad-179.9(4)$

$\mathrm{O}(4) \quad \mathrm{C}(10) \quad \mathrm{N}(1) \quad \mathrm{C}(9) \quad-146.1(4)$

$\mathrm{O}(4) \quad \mathrm{C}(10) \quad \mathrm{C}(1) \quad \mathrm{C}(2) \quad-20.3(6)$

$\mathrm{N}(1) \quad \mathrm{C}(9) \quad \mathrm{C}(8) \quad \mathrm{C}(7) \quad-90.7(4)$

$N(1) \quad C(9) \quad C(11) \quad C(16) \quad-59.8(6)$

$\mathrm{N}(1) \quad \mathrm{C}(10) \quad \mathrm{O}(4) \quad \mathrm{C}(24) \quad 51.1(5)$

$\mathrm{N}(1) \quad \mathrm{C}(10) \quad \mathrm{C}(1) \quad \mathrm{C}(6) \quad-71.6(5)$

$\mathrm{N}(1) \quad \mathrm{C}(17) \quad \mathrm{C}(22) \quad \mathrm{C}(21) \quad-178.2(4)$

C(1) $C(6) \quad C(5) \quad C(4) \quad 0.6(7)$

C(1) $\quad \mathrm{C}(10) \quad \mathrm{O}(1) \quad \mathrm{C}(8) \quad-74.7(4)$

C(1) $\quad \mathrm{C}(10) \quad \mathrm{N}(1) \quad \mathrm{C}(9) \quad 87.7(5)$

$C(2) \quad C(1) \quad C(6) \quad C(5) \quad-1.4(7)$

$C(2) \quad C(3) \quad C(4) \quad C(5) \quad-0.6(8)$

$C(3) \quad C(2) \quad C(1) \quad C(10)-179.3(5)$

$C(4) \quad C(5) \quad C(6) \quad C(7) \quad-179.0(5)$

$C(5) \quad C(6) \quad C(7) \quad C(8) \quad 177.1(4)$

$C(7) \quad C(6) \quad C(1) \quad C(10) \quad-1.3(6)$

$C(7) \quad C(8) \quad C(9) \quad C(11) \quad 147.9(4)$

$\mathrm{C}(8) \quad \mathrm{C}(9) \quad \mathrm{N}(1) \quad \mathrm{C}(17) \quad 148.7(4)$

$C(8) \quad C(9) \quad C(11) \quad C(16) \quad 53.7(6)$

$\mathrm{C}(9) \quad \mathrm{N}(1) \quad \mathrm{C}(17) \quad \mathrm{C}(22)-170.1(4)$

$\mathrm{C}(9) \quad \mathrm{C}(11) \quad \mathrm{C}(12) \quad \mathrm{C}(13) \quad 178.6(5)$

$\mathrm{C}(10) \quad \mathrm{N}(1) \quad \mathrm{C}(9) \quad \mathrm{C}(11) \quad 121.7(4)$

C(10) $N(1) \quad C(17) \quad C(22) \quad-25.8(7)$

$C(11) \quad C(12) \quad C(13) \quad C(14) \quad-2.2(9)$

$C(12) \quad C(11) \quad C(16) \quad C(15) \quad 0.5(8)$

C(13) C(12) C(11) C(16) $1.1(8)$

$C(17) \quad C(18) \quad C(19) \quad C(20) \quad 0.2(8)$

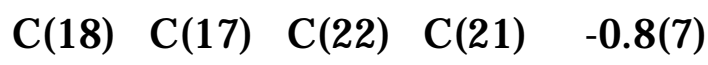

$\begin{array}{lllll}C(19) & C(18) & C(17) & C(22) & 0.6(8)\end{array}$

$C(19) \quad C(20) \quad C(21) \quad C(22) \quad 0.7(8)$ 
$C(21) \quad C(20) \quad O(3) \quad C(23) \quad 179.1(5)$ 
Table 6. Non-bonded Contacts out to $3.60 \AA$

atom atom distance $A D C$ atom atom distance $A D C$

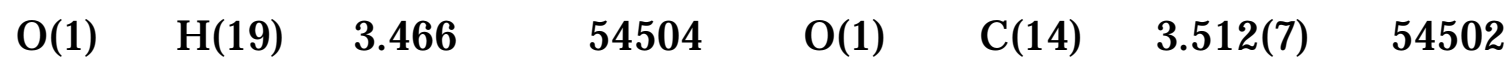

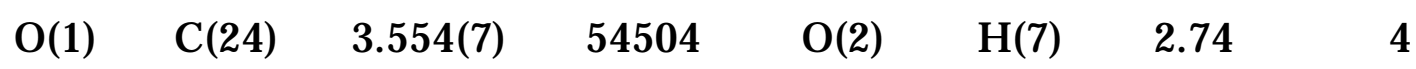

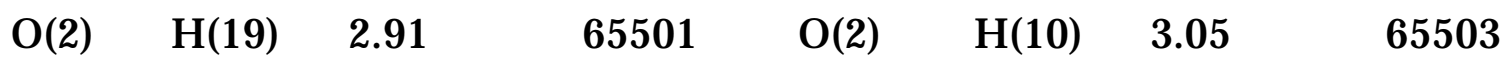

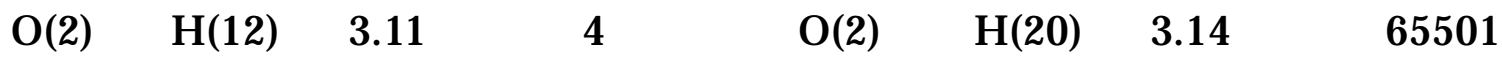

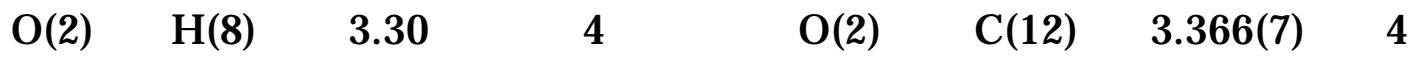

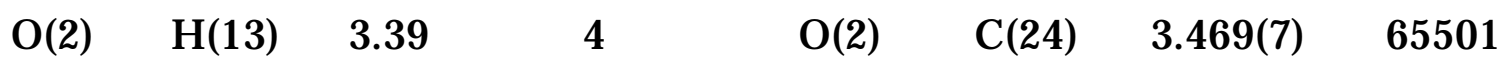

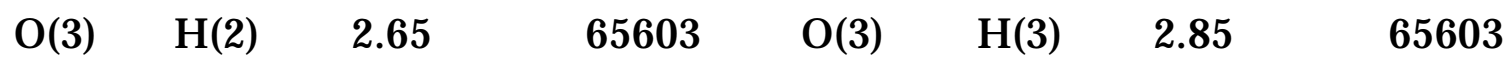

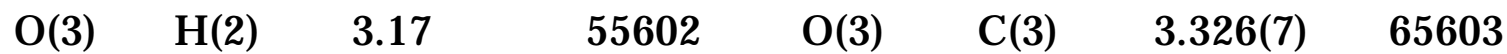

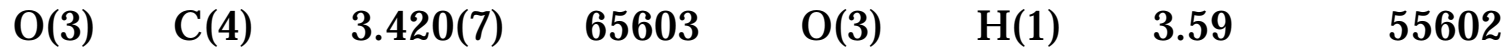

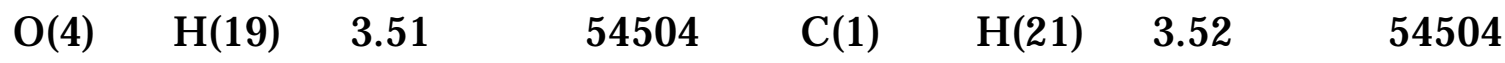

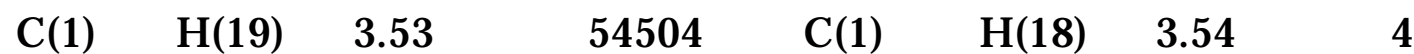

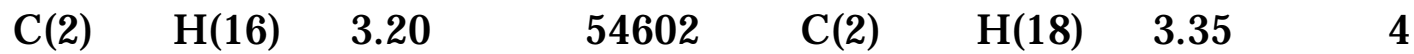

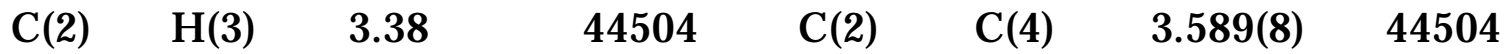

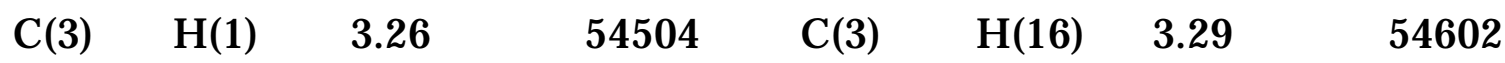

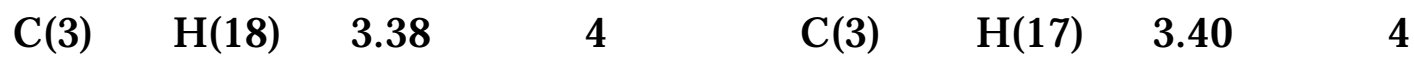

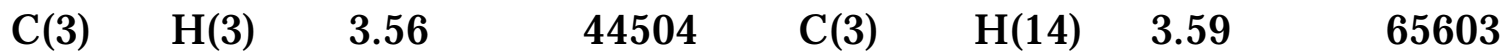

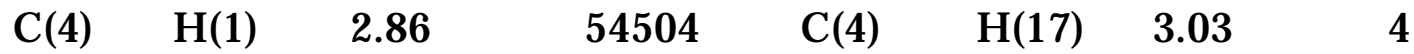

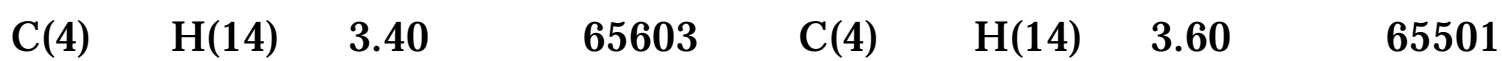

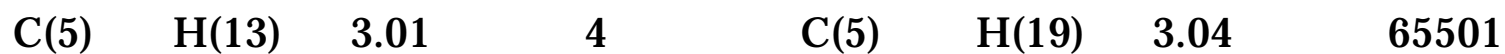

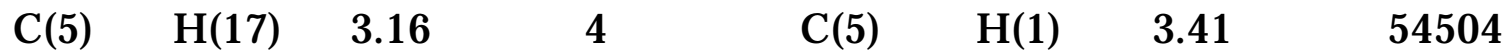

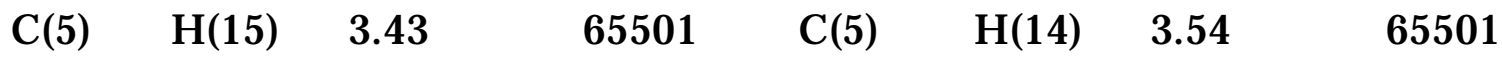

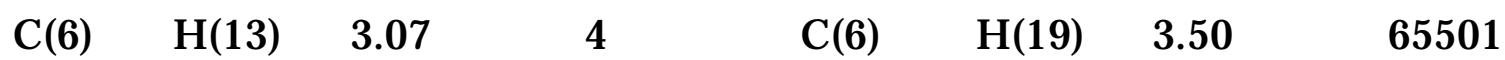

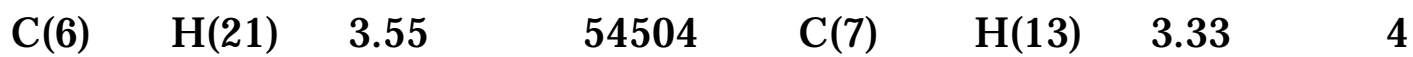

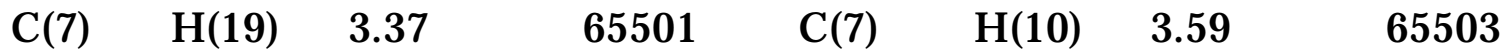

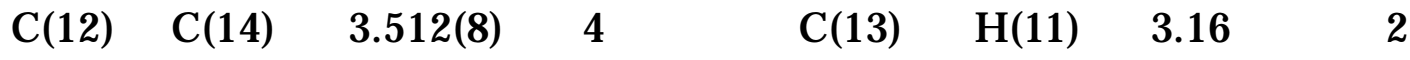

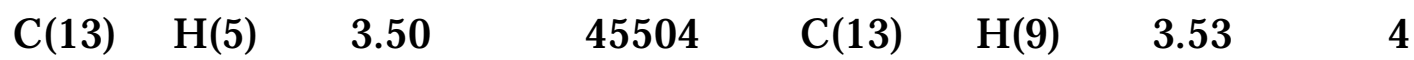

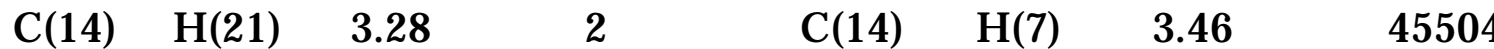

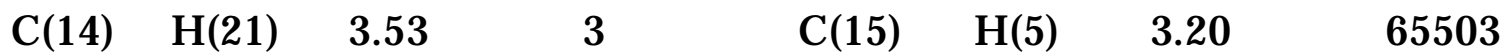

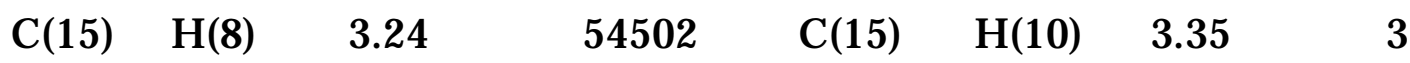

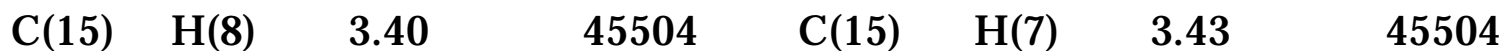

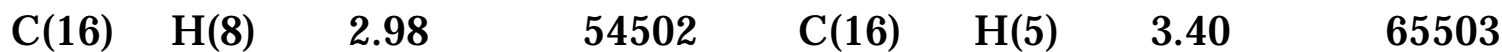

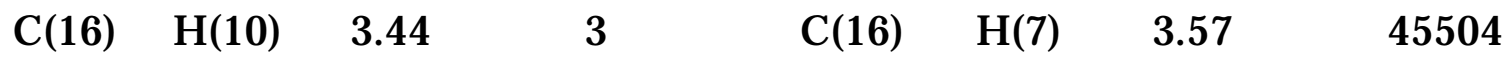

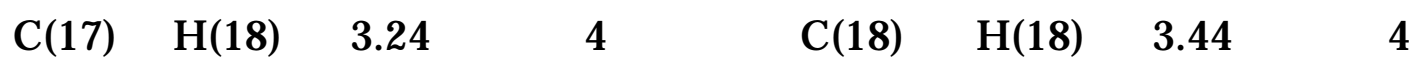

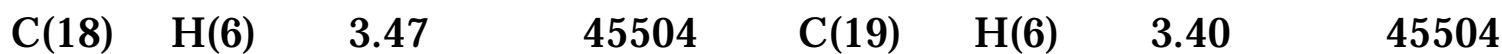

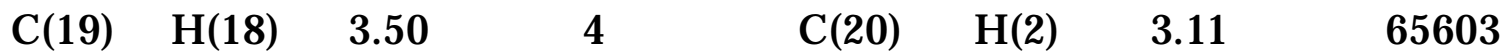

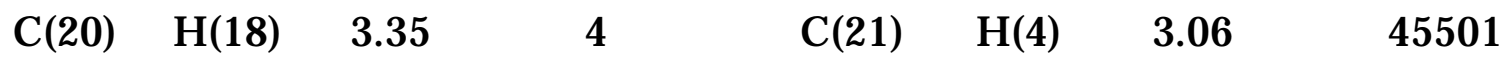




$\begin{array}{llllllll}\mathrm{C}(21) & \mathrm{H}(18) & 3.13 & 4 & \mathrm{C}(21) & \mathrm{H}(2) & 3.36 & 65603 \\ \mathrm{C}(21) & \mathrm{H}(16) & 3.47 & 54602 & \mathrm{C}(21) & \mathrm{H}(3) & 3.52 & 65603 \\ \mathrm{C}(22) & \mathrm{H}(4) & 2.83 & 45501 & \mathrm{C}(22) & \mathrm{H}(18) & 3.08 & 4\end{array}$


Table 6. Non-bonded Contacts out to $3.60 \AA$ (continued)

$\begin{array}{llllllll}\text { atom } & \text { atom } & \text { distance } & \mathrm{ADC} & \text { atom } & \text { atom } & \text { distance } & \mathrm{ADC} \\ \mathrm{C}(22) & \mathrm{H}(16) & 3.56 & 54602 & \mathrm{C}(23) & \mathrm{H}(2) & 3.26 & 55602 \\ \mathrm{C}(23) & \mathrm{H}(2) & 3.32 & 65603 & \mathrm{C}(23) & \mathrm{H}(1) & 3.52 & 55602 \\ \mathrm{C}(24) & \mathrm{H}(4) & 3.32 & 45501 & \mathrm{C}(24) & \mathrm{H}(9) & 3.35 & 3 \\ \mathrm{C}(24) & \mathrm{H}(10) & 3.46 & 3 & \mathrm{C}(24) & \mathrm{H}(9) & 3.49 & 54502 \\ \mathrm{H}(1) & \mathrm{H}(16) & 2.74 & 54602 & \mathrm{H}(1) & \mathrm{H}(3) & 2.77 & 44504 \\ \mathrm{H}(1) & \mathrm{H}(2) & 3.43 & 44504 & \mathrm{H}(2) & \mathrm{H}(16) & 2.91 & 54602 \\ \mathrm{H}(2) & \mathrm{H}(3) & 3.14 & 44504 & \mathrm{H}(2) & \mathrm{H}(18) & 3.19 & 54602 \\ \mathrm{H}(2) & \mathrm{H}(16) & 3.20 & 65603 & \mathrm{H}(2) & \mathrm{H}(14) & 3.20 & 65603 \\ \mathrm{H}(2) & \mathrm{H}(17) & 3.43 & 65603 & \mathrm{H}(3) & \mathrm{H}(14) & 2.84 & 65603 \\ \mathrm{H}(3) & \mathrm{H}(14) & 3.09 & 65501 & \mathrm{H}(3) & \mathrm{H}(17) & 3.25 & 4 \\ \mathrm{H}(3) & \mathrm{H}(15) & 3.56 & 65501 & \mathrm{H}(4) & \mathrm{H}(19) & 2.48 & 65501 \\ \mathrm{H}(4) & \mathrm{H}(15) & 2.56 & 65501 & \mathrm{H}(4) & \mathrm{H}(14) & 2.98 & 65501 \\ \mathrm{H}(4) & \mathrm{H}(13) & 3.03 & 4 & \mathrm{H}(4) & \mathrm{H}(17) & 3.46 & 4 \\ \mathrm{H}(4) & \mathrm{H}(20) & 3.58 & 65501 & \mathrm{H}(5) & \mathrm{H}(8) & 2.94 & 4 \\ \mathrm{H}(5) & \mathrm{H}(10) & 3.15 & 65503 & \mathrm{H}(5) & \mathrm{H}(11) & 3.50 & 65503 \\ \mathrm{H}(5) & \mathrm{H}(9) & 3.53 & 54502 & \mathrm{H}(6) & \mathrm{H}(13) & 3.20 & 4 \\ \mathrm{H}(6) & \mathrm{H}(12) & 3.31 & 4 & \mathrm{H}(8) & \mathrm{H}(11) & 2.32 & 2 \\ \mathrm{H}(8) & \mathrm{H}(10) & 2.84 & 2 & \mathrm{H}(8) & \mathrm{H}(10) & 3.17 & 4 \\ \mathrm{H}(8) & \mathrm{H}(20) & 3.46 & 2 & \mathrm{H}(8) & \mathrm{H}(21) & 3.53 & 2 \\ \mathrm{H}(9) & \mathrm{H}(21) & 2.74 & 3 & \mathrm{H}(9) & \mathrm{H}(21) & 2.93 & 2 \\ \mathrm{H}(9) & \mathrm{H}(11) & 3.30 & 2 & \mathrm{H}(9) & \mathrm{H}(19) & 3.37 & 3 \\ \mathrm{H}(9) & \mathrm{H}(20) & 3.45 & 3 & \mathrm{H}(9) & \mathrm{H}(20) & 3.47 & 2 \\ \mathrm{H}(10) & \mathrm{H}(10) & 2.86 & 3 & \mathrm{H}(10) & \mathrm{H}(20) & 2.94 & 3 \\ \mathrm{H}(10) & \mathrm{H}(11) & 3.02 & 3 & \mathrm{H}(10) & \mathrm{H}(21) & 3.37 & 3 \\ \mathrm{H}(10) & \mathrm{H}(19) & 3.54 & 3 & \mathrm{H}(14) & \mathrm{H}(16) & 3.02 & 54602 \\ \mathrm{H}(14) & \mathrm{H}(17) & 3.42 & 54602 & \mathrm{H}(14) & \mathrm{H}(17) & 3.45 & 45504 \\ \mathrm{H}(14) & \mathrm{H}(18) & 3.56 & 4 & \mathrm{H}(15) & \mathrm{H}(16) & 3.19 & 54602 \\ \mathrm{H}(15) & \mathrm{H}(18) & 3.47 & 4 & \mathrm{H}(16) & \mathrm{H}(18) & 3.36 & 56603 \\ \mathrm{H}(16) & \mathrm{H}(16) & 3.55 & 56603 & & & & \\ & & & & & & & \end{array}$


The ADC (atom designator code) specifies the position of an atom in a crystal. The 5-digit number shown in the table is a composite of three one-digit numbers and one two-digit number: TA (first digit) + TB (second digit) + TC (third digit) + SN (last two digits). TA, TB and TC are the crystal lattice translation digits al ong cell edges $a, b$ and c. A translation digit of 5 indicates the origin unit cell. If TA $=4$, this indicates a translation of one unit cell length along the a-axis in the negative direction. Each translation digit can range in value from 1 to 9 and thus \pm 4 lattice translations from the origin ( $\mathrm{TA}=5, \mathrm{~TB}=5, \mathrm{TC}=5$ ) can be represented.

The SN, or symmetry operator number, refers to the number of the symmetry operator used to generate the coordinates of the target atom. A list of symmetry operators relevant to this structure is given below.

For a given intermolecular contact, the first atom (origin atom) is located in the origin unit cell and its position can be generated using the identity operator ( $\mathrm{SN}=1)$. Thus, the $A D C$ for an origin atom is always 55501. The position of the second atom (target atom) can be generated using the ADC and the coordinates of the atom in the parameter table. For example, an ADC of 47502 refers to the target atom moved through symmetry operator two, then translated -1 cell translations along the a axis, +2 cell translations along the $b$ axis, and 0 cell translations al ong the $c$ axis.

An ADC of 1 indicates an intermolecular contact between two fragments (eg. cation and anion) that reside in the same asymmetric unit.

Symmetry Operators:
(1) $+\mathrm{X}, \quad+\mathrm{Y}, \quad+\mathrm{Z}$
(2) $\quad 1 / 2-X, 1 / 2+Y,-Z$
(3) $\quad-X, \quad-Y, \quad-Z$
(4) $1 / 2+X, 1 / 2-Y,+Z$ 
X-ray Structure Report of endo-3g

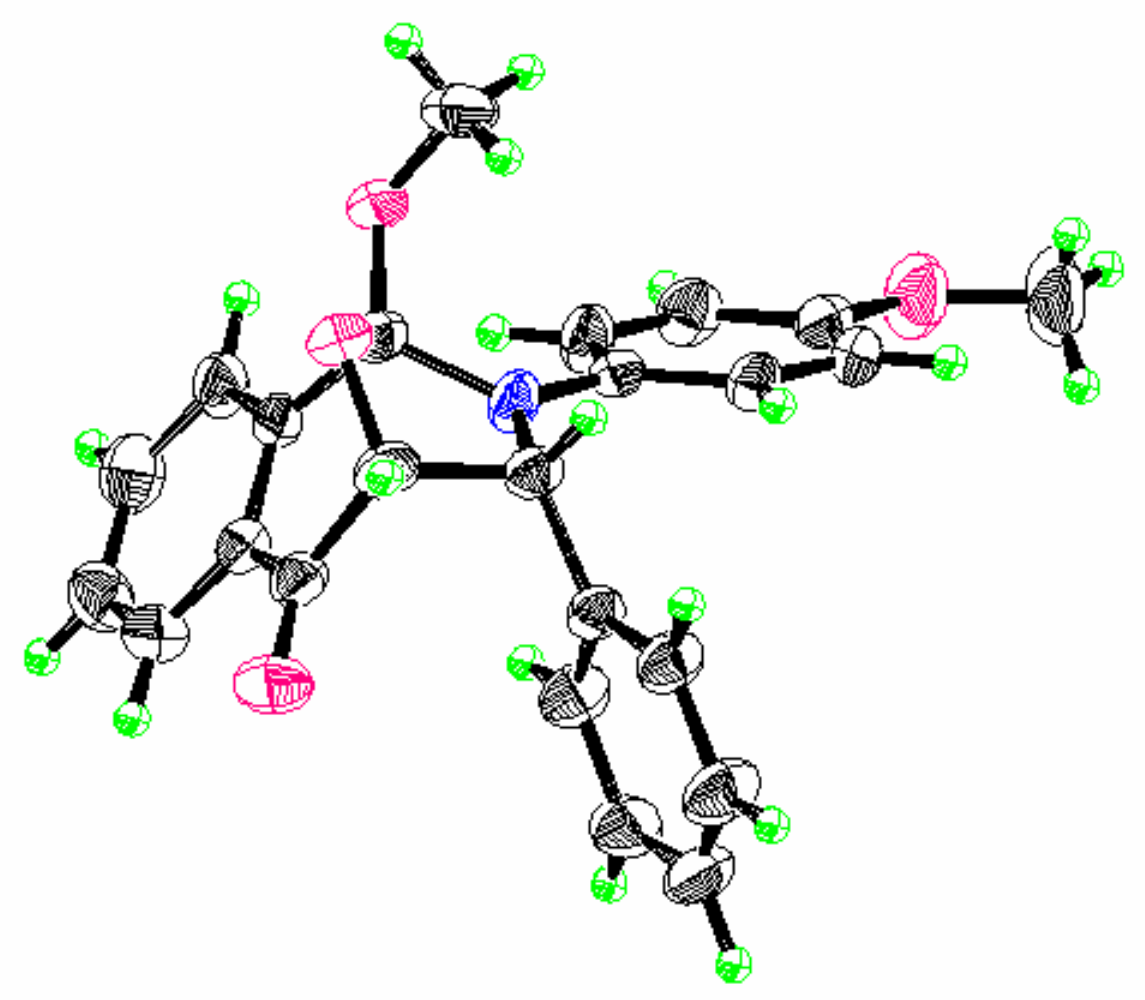

February 2, 2004 


\section{Experimental}

\section{Data Collection}

A col orless prismatic crystal of $\mathrm{C}_{24} \mathrm{H}_{21} \mathrm{NO}_{4}$ having approximate dimensions of $0.24 \times 0.38 \times 0.62 \mathrm{~mm}$ was mounted on a glass fiber. All measurements were made on a Rigaku AFC5S diffractometer with graphite monochromated Mo-K $\alpha$ radiation.

Cell constants and an orientation matrix for data collection, obtained from a least-squares refinement using the setting angles of 25 carefully centered reflections in the range $21.11<2 \theta<24.66^{\circ}$ corresponded to a primitive triclinic cell with dimensions:

$$
\begin{array}{lll}
a=10.501(6) \AA & \alpha=104.10(6)^{\mathrm{O}} \\
\mathrm{b}=11.277(7) \AA & \beta=93.87(6)^{\mathrm{O}} \\
\mathrm{c}=9.106(7) \AA & \gamma=68.20(4)^{\mathrm{O}} \\
\mathrm{V}=971(1) \AA^{3} &
\end{array}
$$

For $Z=2$ and F.W. $=387.43$, the calculated density is $1.33 \mathrm{~g} / \mathrm{cm}^{3}$. Based on a statistical analysis of intensity distribution, and the successful solution and refinement of the structure, the space group was determined to be:

$$
\text { P-1 (\#2) }
$$

The data were collected at a temperature of $23 \pm 10 \mathrm{C}$ using the $\omega-2 \theta$ scan technique to a maximum $2 \theta$ value of $55.0^{\circ}$. Omega scans of several intense reflections, made prior to data collection, had an average width at half-height of 0.080 with a take-off angle of $6.0^{\circ}$. Scans of $(1.37+0.30 \tan \theta)^{\circ}$ were made at a speed of $16.0 \% / \mathrm{min}$ (in $\omega$ ). The weak reflections $(I<10.0 \sigma(I))$ were rescanned (maximum of 3 scans) and the counts were accumulated to ensure good counting statistics. Stationary background counts were recorded on each side of the reflection. The ratio of peak counting time to background counting time was 2:1. The diameter of the incident beam collimator was $1.0 \mathrm{~mm}$, the crystal to detector distance was $258 \mathrm{~mm}$, and the detector aperture was 6.0 x $6.0 \mathrm{~mm}$ (horizontal x vertical).

\section{Data Reduction}

Of the 4699 reflections which were collected, 4458 were unique $\left(R_{\text {int }}=0.027\right)$. 
The intensities of three representative reflections were measured after every 150 reflections. No decay correction was applied.

The linear absorption coefficient, $\mu$, for Mo-K $\alpha$ radiation is $0.9 \mathrm{~cm}^{-1}$. The data were corrected for Lorentz and polarization effects.

\section{$\underline{\text { Structure Solution and Refinement }}$}

The structure was solved by direct methods 1 and expanded using Fourier techniques ${ }^{2}$. The non-hydrogen atoms were refined anisotropically. Hydrogen atoms were included but not refined. The final cycle of full-matrix least-squares refinement 3 on $\mathrm{F}$ was based on 2319 observed reflections $(\mathrm{I}>2.00 \sigma(\mathrm{I}))$ and 262 variable parameters and converged (largest parameter shift was 0.00 times its esd) with unweighted and weighted agreement factors of:

$$
\begin{gathered}
\mathrm{R}=\Sigma|| \mathrm{Fo}|-| \mathrm{FC}|| / \Sigma|\mathrm{Fo}|=0.063 \\
\mathrm{R}_{\mathrm{W}}=\left[\Sigma \mathrm{W}(|\mathrm{Fo}|-\mid \mathrm{FC})^{2} / \Sigma \mathrm{WFo}^{2}\right]^{1 / 2}=0.064
\end{gathered}
$$

The standard deviation of an observation of unit weight 4 was 1.90 . The weighting scheme was based on counting statistics and included a factor $(p=0.017)$ to downweight the intense reflections. Plots of $\Sigma w(|F o|-|F c|)^{2}$ versus $|F o|$, reflection order in data collection, $\sin \theta / \lambda$ and various classes of indices showed no unusual trends. The maximum and minimum peaks on the final difference Fourier map corresponded to 0.66 and $-0.61 \mathrm{e}^{-} / \AA^{3}$, respectively.

Neutral atom scattering factors were taken from Cromer and Waber5. Anomalous dispersion effects were included in $\mathrm{Fcal} \mathrm{c}^{6}$; the values for $\Delta \mathrm{f}^{\prime}$ and $\Delta \mathrm{f}^{\prime \prime}$ were those of Creagh and McAuley7. The values for the mass attenuation coefficients are those of Creagh and Hubbell 8 . All calculations were performed using the teXsan 9 crystallographic software package of Molecular Structure Corporation.

\section{References}

(1) SIR92: Altomare, A., Cascarano, M., Giacovazzo, C., Guagliardi, A. (1994). J . Appl. 
Cryst., 26, 343.

(2) DIRDIF 94: Beurskens, P.T., Admiraal, G., Beurskens, G., Bosman, W.P., de Gelder, R., Israel, R. and Smits, J .M.M.(1994). The DIRDIF-94 program system, Technical Report of the Crystallography Laboratory, University of Nijmegen, The Netherlands.

(3) Least Squares function minimized:

$$
\begin{aligned}
& \sum w\left(\left|\mathrm{Fo}_{\mathrm{o}}-\right| \mathrm{F} \mathrm{d}\right)^{2} \text { where } \\
& w=1 /\left[\sigma^{2}(\mathrm{Fo})\right]=\left[\sigma^{2} \mathrm{c}(\mathrm{Fo})+\mathrm{p}^{2} \mathrm{Fo}^{2} / 4\right]^{-1} \\
& \sigma_{\mathrm{C}}(\mathrm{Fo})=\text { e.s.d. based on counting statistics } \\
& p=\mathrm{p} \text {-factor }
\end{aligned}
$$

(4) Standard deviation of an observation of unit weight:

$$
\begin{aligned}
& {\left[\Sigma m\left(\left|F_{o}\right|-\mid F_{c}\right)^{2} /\left(N_{0}-N_{v}\right)\right]^{1 / 2}} \\
& \text { where: } \quad \mathrm{N}_{\mathrm{O}}=\text { number of observations } \\
& \mathrm{N}_{\mathrm{V}} \text { = number of variables }
\end{aligned}
$$

(5) Cromer, D. T. \& Waber, J . T.; "I nternational Tables for X-ray Crystallography", Vol. IV, The Kynoch Press, Birmingham, England, Table 2.2 A (1974).

(6) I bers, J . A. \& Hamilton, W. C.; Acta Crystallogr., 17, 781 (1964).

(7) Creagh, D. C. \& McAuley, W.J .; "International Tables for Crystallography", Vol C, (A.J .C. Wilson, ed.), Kluwer Academic Publishers, Boston, Table 4.2.6.8, pages 219-222 (1992).

(8) Creagh, D. C. \& Hubbell, J .H..; "International Tables for Crystallography", Vol C, (A.J .C. Wilson, ed.), Kluwer Academic Publishers, Boston, Table 4.2.4.3, pages 200-206 (1992).

(9) teXsan for Windows version 1.06: Crystal Structure Analysis Package, Molecular 
Structure Corporation (1997-9). 
A. Crystal Data

Empirical Formula

Formula Weight

Crystal Color, Habit

Crystal Dimensions

Crystal System

Lattice Type

No. of Reflections Used for Unit

Cell Determination (2 $\theta$ range)

Omega Scan Peak Width

at Half-height

Lattice Parameters

Space Group

$Z$ value

$D_{\text {calc }}$

$\mathrm{F}_{000}$

$\mu(\mathrm{MoK} \alpha)$
$\mathrm{C}_{24} \mathrm{H}_{21} \mathrm{NO}_{4}$

387.43

colorless, prismatic

$0.24 \times 0.38 \times 0.62 \mathrm{~mm}$

triclinic

Primitive

$25(21.1-24.70)$

$0.08^{\circ}$

$a=10.501(6) \AA$

$\mathrm{b}=11.277(7) \AA$

$\mathrm{c}=9.106(7) \AA$

$\alpha=104.10(6) \circ$

$\beta=93.87(6)^{\circ}$

$\gamma=68.20(4) \circ$

$V=971(1) \AA^{3}$

P-1 (\#2)

2

$1.325 \mathrm{~g} / \mathrm{cm}^{3}$

408.00

$0.90 \mathrm{~cm}^{-1}$ 
B. Intensity M easurements

Diffractometer

Radiation

Attenuator

Take-off Angle

Detector Aperture

Crystal to Detector Distance

Voltage, Current

Temperature

Scan Type

Scan Rate

Scan Width

$2 \theta \max$

No. of Reflections Measured

Corrections
Rigaku AFC5S

$\operatorname{MoK} \alpha(\lambda=0.71069 \AA)$

graphite monochromated

Zr foil (factors $=1.00,2.26,5.12,11.58$ )

6.00

$6.0 \mathrm{~mm}$ horizontal

$6.0 \mathrm{~mm}$ vertical

$258 \mathrm{~mm}$

$50 \mathrm{kV}, 30 \mathrm{~mA}$

$23.0^{\circ} \mathrm{C}$

$\omega-2 \theta$

16.0\%/min (in $\omega$ ) (up to 3 scans)

$(1.37+0.30 \tan \theta)^{\mathrm{O}}$

$55.0^{\circ}$

Total : 4699

Unique: $4458\left(\mathrm{R}_{\mathrm{int}}=0.027\right)$

Lorentz-polarization 
C. Structure Solution and Refinement

Structure Solution

Refinement

Function Minimized

Least Squares Weights

p-factor

Anomalous Dispersion

No. Observations $(I>2.00 \sigma(I))$

No. Variables

Reflection/Parameter Ratio

Residuals: R; Rw

Goodness of Fit Indicator

Max Shift/Error in Final Cycle

Maximum peak in Final Diff. Map

Minimum peak in Final Diff. Map
Direct Methods (SIR92)

Full-matrix least-squares on $\mathrm{F}$

$\Sigma w(|F o|-|F c|)^{2}$

$1 / \sigma^{2}(\mathrm{Fo})=4 \mathrm{Fo}^{2} / \sigma^{2}\left(\mathrm{Fo}^{2}\right)$

0.0174

All non-hydrogen atoms

2319

262

8.85

$0.063 ; 0.064$

1.90

0.00

$0.66 \mathrm{e}^{-} / \AA^{3}$

$-0.61 e^{-} / \AA^{3}$ 
Table 1. Atomic coordinates and $\mathrm{B}_{\mathrm{iso}} / \mathrm{B}_{\mathrm{eq}}$

$\begin{array}{lllll}\text { atom } & \mathrm{x} & \mathrm{y} & \mathrm{z} & \mathrm{B} \text { eq } \\ \mathrm{O}(1) & 0.9556(2) & 0.1585(2) & 0.7305(2) & 2.77(4) \\ \mathrm{O}(2) & 0.9519(2) & 0.2033(2) & 1.1222(2) & 3.97(5) \\ \mathrm{O}(3) & 0.1988(2) & 0.3745(2) & 0.5104(3) & 5.05(6) \\ \mathrm{O}(4) & 0.8286(2) & 0.2851(2) & 0.5701(2) & 3.01(5) \\ \mathrm{N}(1) & 0.7245(2) & 0.2166(2) & 0.7447(2) & 2.74(5) \\ \mathrm{C}(1) & 0.8284(3) & 0.3841(3) & 0.8307(3) & 2.37(6) \\ \mathrm{C}(2) & 0.7913(3) & 0.5078(3) & 0.8054(3) & 3.06(7) \\ \mathrm{C}(3) & 0.7882(3) & 0.6125(3) & 0.9245(4) & 3.99(8) \\ \mathrm{C}(4) & 0.8216(3) & 0.5950(3) & 1.0677(4) & 4.02(8) \\ \mathrm{C}(5) & 0.8626(3) & 0.4705(3) & 1.0950(3) & 3.63(7) \\ \mathrm{C}(6) & 0.8670(3) & 0.3646(3) & 0.9771(3) & 2.67(6) \\ \mathrm{C}(7) & 0.9158(3) & 0.2294(3) & 0.9999(3) & 2.79(6) \\ \mathrm{C}(8) & 0.9250(3) & 0.1203(3) & 0.8590(3) & 2.83(6) \\ \mathrm{C}(9) & 0.7838(3) & 0.1090(3) & 0.8185(3) & 2.62(6) \\ \mathrm{C}(10) & 0.8316(3) & 0.2626(3) & 0.7127(3) & 2.65(6) \\ \mathrm{C}(11) & 0.7020(3) & 0.1139(3) & 0.9527(3) & 2.56(6) \\ \mathrm{C}(12) & 0.6200(3) & 0.2322(3) & 1.0456(3) & 3.44(7) \\ \mathrm{C}(13) & 0.5553(3) & 0.2314(3) & 1.1731(3) & 3.86(8) \\ \mathrm{C}(14) & 0.5690(3) & 0.1152(4) & 1.2064(3) & 4.31(9) \\ \mathrm{C}(15) & 0.6489(4) & -0.0019(3) & 1.1134(4) & 4.48(9) \\ \mathrm{C}(16) & 0.7143(3) & -0.0018(3) & 0.9870(3) & 3.33(7) \\ \mathrm{C}(17) & 0.5934(3) & 0.2524(3) & 0.6837(3) & 2.47(6) \\ \mathrm{C}(18) & 0.5212(3) & 0.1676(3) & 0.6550(3) & 2.90(7) \\ \mathrm{C}(19) & 0.3904(3) & 0.2033(3) & 0.5964(3) & 3.20(7) \\ \mathrm{C}(20) & 0.3290(3) & 0.3256(3) & 0.5652(3) & 3.24(7) \\ \mathrm{C}(21) & 0.3993(3) & 0.4109(3) & 0.5933(3) & 3.50(7) \\ \mathrm{C}(22) & 0.5282(3) & 0.3763(3) & 0.6518(3) & 3.22(7) \\ \mathrm{C}(23) & 0.1243(4) & 0.2903(4) & 0.4720(4) & 6.6(1) \\ \mathrm{C}(24) & 0.8449(3) & 0.1730(3) & 0.4469(3) & 4.28(8) \\ \mathrm{H}(1) & 0.7680 & 0.5215 & 0.7066 & 3.674 \\ \mathrm{H}(2) & 0.7625 & 0.6977 & 0.9065 & 4.790 \\ \mathrm{H}(3) & 0.8167 & 0.6682 & 1.1485 & 4.826 \\ \mathrm{H}(4) & 0.8875 & 0.4579 & 1.1939 & 4.357 \\ \mathrm{H}(5) & 0.9918 & 0.0386 & 0.8691 & 3.400 \\ \mathrm{H}(6) & 0.7976 & 0.0287 & 0.7457 & 3.150 \\ \mathrm{H}(7) & 0.6082 & 0.3131 & 1.0219 & 4.127 \\ \mathrm{H}(8) & 0.5009 & 0.3123 & 1.2380 & 4.635\end{array}$




$\begin{array}{llrrr}H(9) & 0.5236 & 0.1155 & 1.2934 & 5.177 \\ H(10) & 0.6590 & -0.0827 & 1.1362 & 5.379 \\ H(11) & 0.7687 & -0.0830 & 0.9227 & 3.998\end{array}$


Table 1. Atomic coordinates and $\mathrm{B}_{\mathrm{iso}} / \mathrm{B}_{\mathrm{eq}}$ (continued)

$\begin{array}{lcccl}\text { atom } & x & y & z & B_{\text {eq }} \\ \mathrm{H}(12) & 0.5626 & 0.0831 & 0.6761 & 3.483 \\ \mathrm{H}(13) & 0.3434 & 0.1438 & 0.5779 & 3.846 \\ \mathrm{H}(14) & 0.3574 & 0.4951 & 0.5716 & 4.198 \\ \mathrm{H}(15) & 0.5739 & 0.4369 & 0.6709 & 3.866 \\ \mathrm{H}(16) & 0.1040 & 0.2702 & 0.5610 & 7.900 \\ \mathrm{H}(17) & 0.0412 & 0.3326 & 0.4261 & 7.900 \\ \mathrm{H}(18) & 0.1775 & 0.2115 & 0.4031 & 7.900 \\ \mathrm{H}(19) & 0.8541 & 0.0995 & 0.4859 & 5.134 \\ \mathrm{H}(20) & 0.7668 & 0.1915 & 0.3850 & 5.134 \\ \mathrm{H}(21) & 0.9247 & 0.1538 & 0.3883 & 5.134\end{array}$

$B_{\text {eq }}=8 / 3 \pi^{2}\left(U_{11}\left(a a^{*}\right)^{2}+U_{22}\left(b b^{*}\right)^{2}+U_{33}\left(c c^{*}\right)^{2}+2 U_{12}\left(a a^{*} b b^{*}\right) \cos \gamma+2 U_{13}\left(a a^{*} c c^{*}\right) \cos \beta+2 U_{23}\left(b b^{*} c c^{*}\right) \cos \alpha\right)$ 
Table 2. Anisotropic Displacement Parameters

$\begin{array}{lllllll}\text { atom } & U_{11} & U_{22} & U_{33} & U_{12} & U_{13} & U_{23} \\ \mathrm{O}(1) & 0.036(1) & 0.036(1) & 0.031(1) & -0.0089(9) & 0.0063(8) & 0.0105(9) \\ \mathrm{O}(2) & 0.061(1) & 0.059(1) & 0.035(1) & -0.019(1) & -0.006(1) & 0.024(1) \\ \mathrm{O}(3) & 0.045(1) & 0.089(2) & 0.076(2) & -0.036(1) & -0.020(1) & 0.038(1) \\ \mathrm{O}(4) & 0.050(1) & 0.047(1) & 0.028(1) & -0.025(1) & -0.0004(9) & 0.0147(9) \\ \mathrm{N}(1) & 0.036(1) & 0.039(1) & 0.041(1) & -0.021(1) & -0.006(1) & 0.020(1) \\ \mathrm{C}(1) & 0.026(1) & 0.033(2) & 0.033(1) & -0.013(1) & 0.000(1) & 0.007(1) \\ \mathrm{C}(2) & 0.044(2) & 0.038(2) & 0.043(2) & -0.023(1) & -0.008(1) & 0.017(1) \\ \mathrm{C}(3) & 0.050(2) & 0.040(2) & 0.065(2) & -0.019(2) & -0.002(2) & 0.013(2) \\ \mathrm{C}(4) & 0.056(2) & 0.045(2) & 0.048(2) & -0.023(2) & 0.004(2) & -0.005(2) \\ \mathrm{C}(5) & 0.049(2) & 0.055(2) & 0.035(2) & -0.022(2) & 0.002(1) & 0.007(2) \\ \mathrm{C}(6) & 0.032(2) & 0.041(2) & 0.032(2) & -0.017(1) & 0.002(1) & 0.009(1) \\ \mathrm{C}(7) & 0.029(1) & 0.046(2) & 0.034(2) & -0.014(1) & 0.002(1) & 0.012(1) \\ \mathrm{C}(8) & 0.037(2) & 0.034(2) & 0.037(2) & -0.008(1) & 0.004(1) & 0.016(1) \\ \mathrm{C}(9) & 0.040(2) & 0.030(1) & 0.031(1) & -0.014(1) & 0.004(1) & 0.008(1) \\ \mathrm{C}(10) & 0.035(2) & 0.042(2) & 0.030(1) & -0.017(1) & -0.001(1) & 0.014(1) \\ \mathrm{C}(11) & 0.035(2) & 0.034(2) & 0.033(2) & -0.013(1) & -0.001(1) & 0.013(1) \\ \mathrm{C}(12) & 0.047(2) & 0.038(2) & 0.048(2) & -0.012(1) & 0.004(1) & 0.016(2) \\ \mathrm{C}(13) & 0.042(2) & 0.055(2) & 0.041(2) & -0.014(2) & 0.008(1) & 0.002(2) \\ \mathrm{C}(14) & 0.051(2) & 0.083(3) & 0.043(2) & -0.032(2) & 0.005(2) & 0.024(2) \\ \mathrm{C}(15) & 0.070(2) & 0.059(2) & 0.056(2) & -0.029(2) & 0.005(2) & 0.028(2) \\ \mathrm{C}(16) & 0.050(2) & 0.036(2) & 0.046(2) & -0.017(1) & 0.005(1) & 0.015(1) \\ \mathrm{C}(17) & 0.037(2) & 0.036(2) & 0.025(1) & -0.017(1) & 0.001(1) & 0.010(1) \\ \mathrm{C}(18) & 0.047(2) & 0.034(2) & 0.038(2) & -0.023(1) & 0.002(1) & 0.011(1) \\ \mathrm{C}(19) & 0.049(2) & 0.049(2) & 0.037(2) & -0.033(2) & -0.001(1) & 0.010(1) \\ \mathrm{C}(20) & 0.038(2) & 0.061(2) & 0.033(2) & -0.025(2) & -0.002(1) & 0.017(2) \\ \mathrm{C}(21) & 0.045(2) & 0.043(2) & 0.049(2) & -0.017(1) & -0.003(1) & 0.019(2) \\ \mathrm{C}(22) & 0.042(2) & 0.041(2) & 0.051(2) & -0.023(1) & -0.007(1) & 0.020(1) \\ \mathrm{C}(23) & 0.065(3) & 0.125(4) & 0.092(3) & -0.065(3) & -0.036(2) & 0.043(3) \\ \mathrm{C}(24) & 0.075(2) & 0.066(2) & 0.028(2) & -0.033(2) & 0.003(2) & 0.011(2)\end{array}$

The general temperature factor expression:exp $\left(-2 \pi^{2}\left(a * 2 U_{11} h^{2}+b^{2} U_{22} k^{2}+\left.c^{2} U_{33}\right|^{2}\right.\right.$ $\left.\left.+2 \mathrm{a} * \mathrm{~b} * \mathrm{U}_{12} \mathrm{hk}+2 \mathrm{a} * \mathrm{c} * \mathrm{U}_{13} \mathrm{hl}+2 \mathrm{~b} * \mathrm{c} * \mathrm{U}_{23} \mathrm{kl}\right)\right)$ 
Table 3. Bond Lengths $(\AA)$

$\begin{array}{llllll}\text { atom } & \text { atom } & \text { distance } & \text { atom } & \text { atom } & \text { distance } \\ \mathrm{O}(1) & \mathrm{C}(8) & 1.434(4) & \mathrm{O}(1) & \mathrm{C}(10) & 1.424(4) \\ \mathrm{O}(2) & \mathrm{C}(7) & 1.221(4) & \mathrm{O}(3) & \mathrm{C}(20) & 1.380(4) \\ \mathrm{O}(3) & \mathrm{C}(23) & 1.410(5) & \mathrm{O}(4) & \mathrm{C}(10) & 1.379(4) \\ \mathrm{O}(4) & \mathrm{C}(24) & 1.436(5) & \mathrm{N}(1) & \mathrm{C}(9) & 1.446(4) \\ \mathrm{N}(1) & \mathrm{C}(10) & 1.473(4) & \mathrm{N}(1) & \mathrm{C}(17) & 1.400(4) \\ \mathrm{C}(1) & \mathrm{C}(2) & 1.375(5) & \mathrm{C}(1) & \mathrm{C}(6) & 1.413(5) \\ \mathrm{C}(1) & \mathrm{C}(10) & 1.512(5) & \mathrm{C}(2) & \mathrm{C}(3) & 1.387(5) \\ \mathrm{C}(2) & \mathrm{H}(1) & 0.95 & \mathrm{C}(3) & \mathrm{C}(4) & 1.368(6) \\ \mathrm{C}(3) & \mathrm{H}(2) & 0.95 & \mathrm{C}(4) & \mathrm{C}(5) & 1.388(6) \\ \mathrm{C}(4) & \mathrm{H}(3) & 0.95 & \mathrm{C}(5) & \mathrm{C}(6) & 1.386(5) \\ \mathrm{C}(5) & \mathrm{H}(4) & 0.95 & \mathrm{C}(6) & \mathrm{C}(7) & 1.480(5) \\ \mathrm{C}(7) & \mathrm{C}(8) & 1.526(5) & \mathrm{C}(8) & \mathrm{C}(9) & 1.547(5) \\ \mathrm{C}(8) & \mathrm{H}(5) & 0.95 & \mathrm{C}(9) & \mathrm{C}(11) & 1.518(5) \\ \mathrm{C}(9) & \mathrm{H}(6) & 0.95 & \mathrm{C}(11) & \mathrm{C}(12) & 1.390(5) \\ \mathrm{C}(11) & \mathrm{C}(16) & 1.373(5) & \mathrm{C}(12) & \mathrm{C}(13) & 1.388(5) \\ \mathrm{C}(12) & \mathrm{H}(7) & 0.95 & \mathrm{C}(13) & \mathrm{C}(14) & 1.370(6) \\ \mathrm{C}(13) & \mathrm{H}(8) & 0.95 & \mathrm{C}(14) & \mathrm{C}(15) & 1.376(6) \\ \mathrm{C}(14) & \mathrm{H}(9) & 0.95 & \mathrm{C}(15) & \mathrm{C}(16) & 1.380(5) \\ \mathrm{C}(15) & \mathrm{H}(10) & 0.95 & \mathrm{C}(16) & \mathrm{H}(11) & 0.95 \\ \mathrm{C}(17) & \mathrm{C}(18) & 1.396(5) & \mathrm{C}(17) & \mathrm{C}(22) & 1.402(5) \\ \mathrm{C}(18) & \mathrm{C}(19) & 1.388(5) & \mathrm{C}(18) & \mathrm{H}(12) & 0.95 \\ \mathrm{C}(19) & \mathrm{C}(20) & 1.380(5) & \mathrm{C}(19) & \mathrm{H}(13) & 0.95 \\ \mathrm{C}(20) & \mathrm{C}(21) & 1.385(5) & \mathrm{C}(21) & \mathrm{C}(22) & 1.371(5) \\ \mathrm{C}(21) & \mathrm{H}(14) & 0.95 & \mathrm{C}(22) & \mathrm{H}(15) & 0.95 \\ \mathrm{C}(23) & \mathrm{H}(16) & 0.95 & \mathrm{C}(23) & \mathrm{H}(17) & 0.95 \\ \mathrm{C}(23) & \mathrm{H}(18) & 0.95 & \mathrm{C}(24) & \mathrm{H}(19) & 0.95 \\ \mathrm{C}(24) & \mathrm{H}(20) & 0.95 & \mathrm{C}(24) & \mathrm{H}(21) & 0.95\end{array}$


Table 4. Bond Angles( $\left.{ }^{\circ}\right)$

\begin{tabular}{|c|c|c|c|c|c|c|c|}
\hline atom & atom & atom & angle & atom & atom & atom & angle \\
\hline$C(8)$ & $\mathrm{O}(1)$ & $C(10)$ & $103.1(3)$ & $C(20)$ & $\mathrm{O}(3)$ & $C(23)$ & $118.0(4)$ \\
\hline$C(10)$ & $\mathrm{O}(4)$ & $C(24)$ & $115.2(3)$ & $C(9)$ & $N(1)$ & $C(10)$ & 109.8(3) \\
\hline$C(9)$ & $N(1)$ & $C(17)$ & $124.7(3)$ & $C(10)$ & $N(1)$ & $C(17)$ & $124.2(3)$ \\
\hline$C(2)$ & $C(1)$ & $C(6)$ & 119.5(3) & $C(2)$ & $C(1)$ & $C(10)$ & $125.3(3)$ \\
\hline$C(6)$ & $C(1)$ & $C(10)$ & $115.3(3)$ & $C(1)$ & $C(2)$ & $C(3)$ & $119.8(4)$ \\
\hline$C(1)$ & $C(2)$ & $H(1)$ & 120 & $C(3)$ & $C(2)$ & $H(1)$ & 120 \\
\hline$C(2)$ & $C(3)$ & C(4) & $121.1(4)$ & $C(2)$ & $C(3)$ & $H(2)$ & 119 \\
\hline$C(4)$ & $C(3)$ & $H(2)$ & 119 & $C(3)$ & $C(4)$ & $C(5)$ & $120.0(4)$ \\
\hline$C(3)$ & $C(4)$ & $\mathrm{H}(3)$ & 119 & $C(5)$ & $C(4)$ & $\mathrm{H}(3)$ & 119 \\
\hline$C(4)$ & $C(5)$ & $C(6)$ & $119.7(4)$ & $C(4)$ & $C(5)$ & $\mathrm{H}(4)$ & 120 \\
\hline$C(6)$ & $C(5)$ & $\mathrm{H}(4)$ & 120 & $C(1)$ & $C(6)$ & $C(5)$ & $119.9(4)$ \\
\hline$C(1)$ & $C(6)$ & $C(7)$ & $118.7(3)$ & $C(5)$ & $C(6)$ & $C(7)$ & $121.4(3)$ \\
\hline $\mathrm{O}(2)$ & $C(7)$ & $C(6)$ & $123.2(4)$ & $\mathrm{O}(2)$ & $C(7)$ & $C(8)$ & $120.6(4)$ \\
\hline$C(6)$ & $C(7)$ & $C(8)$ & $116.2(3)$ & $\mathrm{O}(1)$ & $C(8)$ & $C(7)$ & $108.4(3)$ \\
\hline $\mathrm{O}(1)$ & $C(8)$ & $C(9)$ & 101.9(3) & $\mathrm{O}(1)$ & $C(8)$ & $H(5)$ & 111 \\
\hline$C(7)$ & $C(8)$ & $C(9)$ & $112.0(3)$ & $C(7)$ & $C(8)$ & $H(5)$ & 111 \\
\hline$C(9)$ & $C(8)$ & $H(5)$ & 111 & $N(1)$ & $C(9)$ & $C(8)$ & $99.9(3)$ \\
\hline$N(1)$ & $C(9)$ & $C(11)$ & $115.9(3)$ & $N(1)$ & $C(9)$ & $H(6)$ & 108 \\
\hline$C(8)$ & $C(9)$ & $C(11)$ & $114.4(3)$ & $C(8)$ & $C(9)$ & $H(6)$ & 108 \\
\hline$C(11)$ & $C(9)$ & $H(6)$ & 108 & $\mathrm{O}(1)$ & $C(10)$ & $\mathrm{O}(4)$ & $110.3(3)$ \\
\hline$O(1)$ & $C(10)$ & $N(1)$ & 103.2(3) & $\mathrm{O}(1)$ & $C(10)$ & $C(1)$ & $107.3(3)$ \\
\hline O(4) & $C(10)$ & $N(1)$ & $114.9(3)$ & $\mathrm{O}(4)$ & $C(10)$ & $C(1)$ & 109.6(3) \\
\hline$N(1)$ & $C(10)$ & $C(1)$ & $111.2(3)$ & $C(9)$ & $C(11)$ & $C(12)$ & $121.9(3)$ \\
\hline$C(9)$ & $C(11)$ & $C(16)$ & $118.9(3)$ & $C(12)$ & $C(11)$ & $C(16)$ & $119.1(3)$ \\
\hline$C(11)$ & $C(12)$ & $C(13)$ & $119.6(4)$ & $C(11)$ & $C(12)$ & $H(7)$ & 120 \\
\hline$C(13)$ & $C(12)$ & $\mathrm{H}(7)$ & 120 & $C(12)$ & $C(13)$ & $C(14)$ & $120.7(4)$ \\
\hline$C(12)$ & $C(13)$ & $\mathrm{H}(8)$ & 119 & $C(14)$ & $C(13)$ & $\mathrm{H}(8)$ & 119 \\
\hline$C(13)$ & $C(14)$ & $C(15)$ & $119.7(4)$ & $C(13)$ & $C(14)$ & $H(9)$ & 120 \\
\hline$C(15)$ & $C(14)$ & $H(9)$ & 120 & $C(14)$ & $C(15)$ & $C(16)$ & $119.9(4)$ \\
\hline$C(14)$ & $C(15)$ & $\mathrm{H}(10)$ & 120 & $C(16)$ & $C(15)$ & $\mathrm{H}(10)$ & 120 \\
\hline$C(11)$ & $C(16)$ & $C(15)$ & $121.0(4)$ & $C(11)$ & $C(16)$ & $\mathrm{H}(11)$ & 119 \\
\hline$C(15)$ & $C(16)$ & $\mathrm{H}(11)$ & 119 & $N(1)$ & $C(17)$ & $C(18)$ & $121.5(3)$ \\
\hline$N(1)$ & $C(17)$ & $C(22)$ & $121.2(3)$ & $C(18)$ & $C(17)$ & $C(22)$ & $117.3(3)$ \\
\hline$C(17)$ & $C(18)$ & $C(19)$ & $121.7(3)$ & $C(17)$ & $C(18)$ & $\mathrm{H}(12)$ & 119 \\
\hline$C(19)$ & $C(18)$ & $\mathrm{H}(12)$ & 119 & $C(18)$ & $C(19)$ & $C(20)$ & $119.8(3)$ \\
\hline$C(18)$ & $C(19)$ & $\mathrm{H}(13)$ & 120 & $C(20)$ & $C(19)$ & $\mathrm{H}(13)$ & 120 \\
\hline O(3) & $C(20)$ & $C(19)$ & $125.7(4)$ & $\mathrm{O}(3)$ & $C(20)$ & $C(21)$ & $115.1(4)$ \\
\hline
\end{tabular}




$\begin{array}{llllllll}\mathrm{C}(19) & \mathrm{C}(20) & \mathrm{C}(21) & 119.2(4) & \mathrm{C}(20) & \mathrm{C}(21) & \mathrm{C}(22) & 121.2(4) \\ \mathrm{C}(20) & \mathrm{C}(21) & \mathrm{H}(14) & 119 & \mathrm{C}(22) & \mathrm{C}(21) & \mathrm{H}(14) & 119 \\ \mathrm{C}(17) & \mathrm{C}(22) & \mathrm{C}(21) & 120.8(4) & \mathrm{C}(17) & \mathrm{C}(22) & \mathrm{H}(15) & 119\end{array}$


Table 4. Bond Angles( ${ }^{\circ}$ (continued)

$\begin{array}{llllllll}\text { atom } & \text { atom } & \text { atom } & \text { angle } & \text { atom } & \text { atom } & \text { atom } & \text { angle } \\ \mathrm{C}(21) & \mathrm{C}(22) & \mathrm{H}(15) & 119 & \mathrm{O}(3) & \mathrm{C}(23) & \mathrm{H}(16) & 109 \\ \mathrm{O}(3) & \mathrm{C}(23) & \mathrm{H}(17) & 109 & \mathrm{O}(3) & \mathrm{C}(23) & \mathrm{H}(18) & 109 \\ \mathrm{H}(16) & \mathrm{C}(23) & \mathrm{H}(17) & 109 & \mathrm{H}(16) & \mathrm{C}(23) & \mathrm{H}(18) & 109 \\ \mathrm{H}(17) & \mathrm{C}(23) & \mathrm{H}(18) & 109 & \mathrm{O}(4) & \mathrm{C}(24) & \mathrm{H}(19) & 109 \\ \mathrm{O}(4) & \mathrm{C}(24) & \mathrm{H}(20) & 109 & \mathrm{O}(4) & \mathrm{C}(24) & \mathrm{H}(21) & 109 \\ \mathrm{H}(19) & \mathrm{C}(24) & \mathrm{H}(20) & 109 & \mathrm{H}(19) & \mathrm{C}(24) & \mathrm{H}(21) & 109 \\ \mathrm{H}(20) & \mathrm{C}(24) & \mathrm{H}(21) & 109 & & & & \end{array}$


Table 5. Torsion Angles( $(0)$

atom atom atom atom angle

$\mathrm{O}(1) \quad \mathrm{C}(8) \quad \mathrm{C}(7) \quad \mathrm{O}(2) \quad-146.8(3)$

$\mathrm{O}(1) \quad \mathrm{C}(8) \quad \mathrm{C}(9) \quad \mathrm{N}(1) \quad-36.4(3)$

$\mathrm{O}(1) \quad \mathrm{C}(10) \mathrm{O}(4) \quad \mathrm{C}(24) \quad 56.1(4)$

$\mathrm{O}(1) \quad \mathrm{C}(10) \quad \mathrm{N}(1) \quad \mathrm{C}(17) \quad-151.9(3)$

$\mathrm{O}(1) \quad \mathrm{C}(10) \quad \mathrm{C}(1) \quad \mathrm{C}(6) \quad-44.2(4)$

$\mathrm{O}(2) \quad \mathrm{C}(7) \quad \mathrm{C}(6) \quad \mathrm{C}(5) \quad 1.6(6)$

$\mathrm{O}(3) \quad \mathrm{C}(20) \quad \mathrm{C}(19) \quad \mathrm{C}(18) \quad-178.4(4)$

$\mathrm{O}(4) \quad \mathrm{C}(10) \quad \mathrm{O}(1) \quad \mathrm{C}(8) \quad-162.5(3)$

$\mathrm{O}(4) \quad \mathrm{C}(10) \quad \mathrm{N}(1) \quad \mathrm{C}(17) \quad-31.9(5)$

$\mathrm{O}(4) \quad \mathrm{C}(10) \quad \mathrm{C}(1) \quad \mathrm{C}(6) \quad-164.0(3)$

$\mathrm{N}(1) \quad \mathrm{C}(9) \quad \mathrm{C}(11) \quad \mathrm{C}(12) \quad-30.8(5)$

$\mathrm{N}(1) \quad \mathrm{C}(10) \quad \mathrm{O}(1) \quad \mathrm{C}(8) \quad-39.3(3)$

$N(1) \quad C(10) \quad C(1) \quad C(2) \quad-111.9(4)$

$\mathrm{N}(1) \quad \mathrm{C}(17) \quad \mathrm{C}(18) \quad \mathrm{C}(19) \quad 179.1(4)$

$C(1) \quad C(2) \quad C(3) \quad C(4) \quad 0.0(6)$

$C(1) \quad C(6) \quad C(7) \quad C(8) \quad 2.1(5)$

$C(1) \quad C(10) \quad O(4) \quad C(24) \quad 174.1(3)$

$\mathrm{C}(1) \quad \mathrm{C}(10) \mathrm{N}(1) \quad \mathrm{C}(17) \quad 93.3(4)$

$C(2) \quad C(1) \quad C(6) \quad C(7) \quad-175.8(3)$

$C(3) \quad C(2) \quad C(1) \quad C(6) \quad-2.0(6)$

$C(3) \quad C(4) \quad C(5) \quad C(6) \quad-1.2(6)$

$C(5) \quad C(6) \quad C(1) \quad C(10)-177.6(3)$

$C(6) \quad C(7) \quad C(8) \quad C(9) \quad-80.9(4)$

$\mathrm{C}(7) \quad \mathrm{C}(8) \quad \mathrm{O}(1) \quad \mathrm{C}(10) \quad-70.5(3)$

$\mathrm{C}(8) \quad \mathrm{C}(9) \quad \mathrm{N}(1) \quad \mathrm{C}(10) \quad 12.8(4)$

C(8) $\quad \mathrm{C}(9) \quad \mathrm{C}(11) \quad \mathrm{C}(12) \quad 84.5(4)$

$\mathrm{C}(9) \quad \mathrm{N}(1) \quad \mathrm{C}(17) \quad \mathrm{C}(18) \quad-16.3(5)$

$\mathrm{C}(9) \quad \mathrm{C}(8) \quad \mathrm{O}(1) \quad \mathrm{C}(10) \quad 47.8(3)$

C(9) $\quad C(11) \quad C(16) \quad C(15) \quad 175.8(4)$

C(10) $\quad \mathrm{N}(1) \quad \mathrm{C}(17) \quad \mathrm{C}(18) \quad 149.1(4)$

C(11) C(9) $\mathrm{N}(1) \quad \mathrm{C}(17) \quad-56.7(5)$

$\mathrm{C}(11) \quad \mathrm{C}(16) \quad \mathrm{C}(15) \quad \mathrm{C}(14) \quad 0.5(7)$

$C(12) \quad C(13) \quad C(14) \quad C(15) \quad 0.7(7)$

$C(13) \quad C(14) \quad C(15) \quad C(16) \quad-0.2(7)$

$C(17) \quad C(22) \quad C(21) \quad C(20) \quad 0.6(6)$

$C(18) \quad C(19) \quad C(20) \quad C(21) \quad-0.1(6)$

$\begin{array}{lllll}C(19) & C(20) & O(3) & C(23) & -4.9(6)\end{array}$ atom atom atom atom angle

$\mathrm{O}(1) \quad \mathrm{C}(8) \quad \mathrm{C}(7) \quad \mathrm{C}(6) \quad 30.8(4)$

O(1) $\quad C(8) \quad C(9) \quad C(11) \quad-160.8(3)$

$\mathrm{O}(1) \quad \mathrm{C}(10) \mathrm{N}(1) \quad \mathrm{C}(9) \quad 15.4(4)$

$\mathrm{O}(1) \quad \mathrm{C}(10) \quad \mathrm{C}(1) \quad \mathrm{C}(2) \quad 135.9(4)$

$\mathrm{O}(2) \quad \mathrm{C}(7) \quad \mathrm{C}(6) \quad \mathrm{C}(1) \quad 179.7(3)$

$\mathrm{O}(2) \quad \mathrm{C}(7) \quad \mathrm{C}(8) \quad \mathrm{C}(9) \quad 101.5(4)$

$\begin{array}{lllll}O(3) & C(20) & C(21) & C(22) & 178.3(4)\end{array}$

$\mathrm{O}(4) \quad \mathrm{C}(10) \quad \mathrm{N}(1) \quad \mathrm{C}(9) \quad 135.5(3)$

$\mathrm{O}(4) \quad \mathrm{C}(10) \quad \mathrm{C}(1) \quad \mathrm{C}(2) \quad 16.1(5)$

$\mathrm{N}(1) \quad \mathrm{C}(9) \quad \mathrm{C}(8) \quad \mathrm{C}(7) \quad 79.3(3)$

$\mathrm{N}(1) \quad \mathrm{C}(9) \quad \mathrm{C}(11) \quad \mathrm{C}(16) \quad 152.1(3)$

$\mathrm{N}(1) \quad \mathrm{C}(10) \quad \mathrm{O}(4) \quad \mathrm{C}(24) \quad-59.9(4)$

$\mathrm{N}(1) \quad \mathrm{C}(10) \quad \mathrm{C}(1) \quad \mathrm{C}(6) \quad 68.0(4)$

$\mathrm{N}(1) \quad \mathrm{C}(17) \quad \mathrm{C}(22) \quad \mathrm{C}(21) \quad-179.4(4)$

C(1) $C(6) \quad C(5) \quad C(4) \quad-0.7(6)$

$C(1) \quad C(10) \quad O(1) \quad C(8) \quad 78.2(3)$

C(1) $\quad \mathrm{C}(10) \quad \mathrm{N}(1) \quad \mathrm{C}(9) \quad-99.4(3)$

$\mathrm{C}(2) \quad \mathrm{C}(1) \quad \mathrm{C}(6) \quad \mathrm{C}(5) \quad 2.3(5)$

C(2) $\quad C(3) \quad C(4) \quad C(5) \quad 1.6(7)$

$C(3) \quad C(2) \quad C(1) \quad C(10) \quad 177.9(4)$

$C(4) \quad C(5) \quad C(6) \quad C(7) \quad 177.3(4)$

$C(5) \quad C(6) \quad C(7) \quad C(8) \quad-176.0(3)$

C(7) $\quad C(6) \quad C(1) \quad C(10) \quad 4.3(5)$

$C(7) \quad C(8) \quad C(9) \quad C(11) \quad-45.1(4)$

$\mathrm{C}(8) \quad \mathrm{C}(9) \quad \mathrm{N}(1) \quad \mathrm{C}(17)-179$

$C(8) \quad C(9) \quad C(11) \quad C(16) \quad-92.6(4)$

$\mathrm{C}(9) \quad \mathrm{N}(1) \quad \mathrm{C}(17) \quad \mathrm{C}(22) \quad 162.4(3)$

$C(9) \quad C(11) \quad C(12) \quad C(13)-175.3(4)$

$C(10) \quad N(1) \quad C(9) \quad C(11) \quad 136.1(3)$

$\mathrm{C}(10) \quad \mathrm{N}(1) \quad \mathrm{C}(17) \quad \mathrm{C}(22) \quad-32.2(6)$

C(11) C(12) C(13) C(14) -1.5(6)

$C(12) \quad C(11) \quad C(16) \quad C(15) \quad-1.3(6)$

C(13) C(12) C(11) C(16) 1.8(6)

$C$ (17) $C(18) \quad C(19) \quad C(20) \quad 0.0(6)$

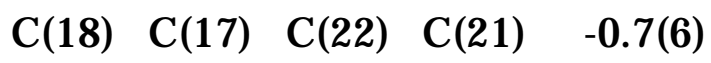

$C(19) \quad C(18) \quad C(17) \quad C(22) \quad 0.4(5)$

$C(19) \quad C(20) \quad C(21) \quad C(22) \quad-0.2(6)$ 
$C(21) \quad C(20) \quad O(3) \quad C(23) \quad 176.8(4)$ 
Table 6. Non-bonded Contacts out to $3.60 \AA$

\begin{tabular}{|c|c|c|c|c|c|c|c|}
\hline atom & atom & distance & ADC & atom & atom & distance & ADC \\
\hline$O(1)$ & $H(16)$ & 3.021 & 65501 & $\mathrm{O}(1)$ & $H(19)$ & 3.125 & 75602 \\
\hline $\mathrm{O}(1)$ & $\mathrm{H}(21)$ & 3.193 & 75602 & $\mathrm{O}(1)$ & $C(24)$ & $3.560(6)$ & 75602 \\
\hline$O(1)$ & $H(3)$ & 3.580 & 76702 & $\mathrm{O}(2)$ & $H(5)$ & 2.588 & 75702 \\
\hline $\mathrm{O}(2)$ & $\mathrm{H}(21)$ & 2.671 & 55601 & $\mathrm{O}(2)$ & $\mathrm{H}(11)$ & 2.749 & 7570 \\
\hline$O(2)$ & $\mathrm{H}(17)$ & 3.060 & 65601 & $\mathrm{O}(2)$ & $\mathrm{H}(20)$ & 3.239 & 556 \\
\hline$O(2)$ & $H(6)$ & 3.348 & 75702 & $\mathrm{O}(2)$ & $C(24)$ & $3.368(5)$ & 556 \\
\hline$O(2)$ & $\mathrm{H}(18)$ & 3.38 & 65601 & $\mathrm{O}(2)$ & $C(8)$ & $3.433(5)$ & \\
\hline$O(2)$ & $C(16)$ & $3.448(6)$ & 75702 & $\mathrm{O}(3)$ & $H(1)$ & 2.63 & 666 \\
\hline$O(3)$ & $H(4)$ & 2.87 & 66702 & $\mathrm{O}(3)$ & $\mathrm{H}(17)$ & 3.27 & $566 C$ \\
\hline $\mathrm{O}(3)$ & $H(3)$ & 3.28 & 66702 & $\mathrm{O}(3)$ & $C(2)$ & $3.470(5)$ & 6660 \\
\hline$O(3)$ & $C(5)$ & $3.578(6)$ & 66702 & $O(4)$ & $H(16)$ & 2.84 & 655 \\
\hline $\mathrm{O}(4)$ & $\mathrm{H}(17)$ & 2.94 & 65501 & $O(4)$ & $H(14)$ & 3.04 & 666 \\
\hline$O(4)$ & $C(23)$ & $3.314(6)$ & 65501 & $C(1)$ & $H(3)$ & 3.55 & 7670 \\
\hline$C(1)$ & $H(16)$ & 3.57 & 65501 & $C(2)$ & $H(8)$ & 3.05 & 667 \\
\hline$C(2)$ & $H(4)$ & 3.53 & 76702 & $C(3)$ & $H(8)$ & 3.20 & 6670 \\
\hline$C(3)$ & $H(10)$ & 3.32 & 56501 & $C(3)$ & $\mathrm{H}(11)$ & 3.37 & 5650 \\
\hline$C(3)$ & $\mathrm{H}(4)$ & 3.39 & 76702 & $C(3)$ & $C(5)$ & $3.437(6)$ & 7670 \\
\hline$C(3)$ & $C(13)$ & $3.538(6)$ & 66702 & $C(4)$ & $\mathrm{H}(10)$ & 3.31 & 5650 \\
\hline$C(4)$ & $C(5)$ & $3.471(6)$ & 76702 & $C(4)$ & $H(16)$ & 3.52 & 6670 \\
\hline$C(4)$ & $C(6)$ & $3.527(6)$ & 76702 & $C(4)$ & $C(20)$ & $3.561(6)$ & 6670 \\
\hline$C(5)$ & $C(20)$ & $3.585(7)$ & 66702 & $C(6)$ & $H(3)$ & 3.45 & 7670 \\
\hline$C(7)$ & $H(11)$ & 3.22 & 75702 & $C(7)$ & $H(5)$ & 3.30 & 757 \\
\hline$C(8)$ & $\mathrm{H}(21)$ & 3.25 & 75602 & $C(8)$ & $\mathrm{H}(5)$ & 3.29 & $757 c$ \\
\hline$C(9)$ & $\mathrm{H}(18)$ & 3.57 & 65602 & $C(10)$ & $H(16)$ & 3.30 & 6550 \\
\hline$C(11)$ & $H(5)$ & 3.53 & 75702 & $C(12)$ & $\mathrm{H}(20)$ & 3.41 & 5560 \\
\hline$C(13)$ & $H(20)$ & 2.82 & 55601 & $C(13)$ & $\mathrm{H}(2)$ & 3.21 & 6670 \\
\hline$C(13)$ & $H(15)$ & 3.43 & 66702 & $C(13)$ & $\mathrm{H}(1)$ & 3.53 & 667 \\
\hline$C(14)$ & $H(20)$ & 2.81 & 55601 & $C(14)$ & $\mathrm{H}(12)$ & 3.42 & 6570 \\
\hline$C(14)$ & $H(2)$ & 3.57 & 66702 & $C(15)$ & $\mathrm{H}(2)$ & 3.26 & 545 \\
\hline$C(15)$ & $H(20)$ & 3.37 & 55601 & $C(15)$ & $\mathrm{H}(12)$ & 3.52 & 6570 \\
\hline$C(15)$ & $H(13)$ & 3.54 & 65702 & $C(15)$ & $\mathrm{H}(3)$ & 3.56 & 545 \\
\hline$C(16)$ & $\mathrm{H}(2)$ & 3.14 & 54501 & $C(16)$ & $\mathrm{H}(5)$ & 3.19 & 7570 \\
\hline$C(18)$ & $H(9)$ & 3.20 & 55401 & $C(18)$ & $H(10)$ & 3.29 & $657 c$ \\
\hline$C(18)$ & $H(13)$ & 3.45 & 65602 & $C(18)$ & $\mathrm{H}(9)$ & 3.54 & 657 \\
\hline$C(19)$ & $H(9)$ & 2.95 & 55401 & $C(19)$ & $\mathrm{H}(3)$ & 2.99 & 667 \\
\hline$C(19)$ & $H(10)$ & 3.22 & 65702 & $C(19)$ & $\mathrm{H}(12)$ & 3.45 & 656 \\
\hline$C(20)$ & $H(3)$ & 3.08 & 66702 & $C(20)$ & $\mathrm{H}(4)$ & 3.14 & \\
\hline
\end{tabular}




$\begin{array}{llllllll}\mathrm{C}(20) & \mathrm{H}(9) & 3.18 & 55401 & \mathrm{C}(20) & \mathrm{H}(1) & 3.25 & 66602 \\ \mathrm{C}(20) & \mathrm{H}(8) & 3.53 & 55401 & \mathrm{C}(21) & \mathrm{H}(1) & 3.24 & 66602 \\ \mathrm{C}(21) & \mathrm{H}(8) & 3.27 & 55401 & \mathrm{C}(21) & \mathrm{H}(4) & 3.33 & 66702\end{array}$


Table 6. Non-bonded Contacts out to $3.60 \AA$ (continued)

$\begin{array}{llllllll}\text { atom } & \text { atom } & \text { distance } & \text { ADC } & \text { atom } & \text { atom } & \text { distance } & \text { ADC } \\ \mathrm{C}(21) & \mathrm{H}(15) & 3.36 & 66602 & \mathrm{C}(22) & \mathrm{H}(14) & 3.28 & 66602 \\ \mathrm{C}(22) & \mathrm{H}(8) & 3.31 & 66702 & \mathrm{C}(23) & \mathrm{H}(21) & 2.99 & 45501 \\ \mathrm{C}(23) & \mathrm{H}(3) & 3.42 & 66702 & \mathrm{C}(23) & \mathrm{H}(6) & 3.48 & 65602 \\ \mathrm{C}(23) & \mathrm{H}(1) & 3.48 & 66602 & \mathrm{C}(23) & \mathrm{H}(4) & 3.59 & 66702 \\ \mathrm{C}(24) & \mathrm{H}(17) & 3.24 & 65501 & \mathrm{C}(24) & \mathrm{H}(16) & 3.31 & 65501 \\ \mathrm{C}(24) & \mathrm{H}(5) & 3.35 & 75602 & \mathrm{C}(24) & \mathrm{H}(14) & 3.59 & 66602 \\ \mathrm{H}(1) & \mathrm{H}(8) & 2.75 & 66702 & \mathrm{H}(1) & \mathrm{H}(14) & 2.77 & 66602 \\ \mathrm{H}(1) & \mathrm{H}(17) & 3.45 & 66602 & \mathrm{H}(2) & \mathrm{H}(11) & 2.47 & 56501 \\ \mathrm{H}(2) & \mathrm{H}(10) & 2.73 & 56501 & \mathrm{H}(2) & \mathrm{H}(8) & 3.01 & 66702 \\ \mathrm{H}(2) & \mathrm{H}(18) & 3.39 & 66602 & \mathrm{H}(2) & \mathrm{H}(4) & 3.51 & 76702 \\ \mathrm{H}(3) & \mathrm{H}(10) & 2.70 & 56501 & \mathrm{H}(3) & \mathrm{H}(16) & 2.72 & 66702 \\ \mathrm{H}(3) & \mathrm{H}(13) & 2.98 & 66702 & \mathrm{H}(4) & \mathrm{H}(17) & 2.89 & 65601 \\ \mathrm{H}(4) & \mathrm{H}(14) & 3.26 & 66702 & \mathrm{H}(4) & \mathrm{H}(16) & 3.35 & 66702 \\ \mathrm{H}(5) & \mathrm{H}(5) & 2.70 & 75702 & \mathrm{H}(5) & \mathrm{H}(21) & 2.71 & 75602 \\ \mathrm{H}(5) & \mathrm{H}(11) & 3.16 & 75702 & \mathrm{H}(5) & \mathrm{H}(20) & 3.39 & 75602 \\ \mathrm{H}(5) & \mathrm{H}(19) & 3.45 & 75602 & \mathrm{H}(6) & \mathrm{H}(18) & 2.64 & 65602 \\ \mathrm{H}(6) & \mathrm{H}(21) & 3.01 & 75602 & \mathrm{H}(7) & \mathrm{H}(15) & 3.51 & 66702 \\ \mathrm{H}(8) & \mathrm{H}(15) & 2.57 & 66702 & \mathrm{H}(8) & \mathrm{H}(20) & 3.00 & 55601 \\ \mathrm{H}(8) & \mathrm{H}(14) & 3.19 & 66702 & \mathrm{H}(8) & \mathrm{H}(14) & 3.32 & 55601 \\ \mathrm{H}(9) & \mathrm{H}(12) & 2.79 & 65702 & \mathrm{H}(9) & \mathrm{H}(20) & 2.99 & 55601 \\ \mathrm{H}(9) & \mathrm{H}(13) & 3.19 & 55601 & \mathrm{H}(9) & \mathrm{H}(13) & 3.21 & 65702 \\ \mathrm{H}(9) & \mathrm{H}(18) & 3.52 & 55601 & \mathrm{H}(9) & \mathrm{H}(12) & 3.57 & 55601 \\ \mathrm{H}(10) & \mathrm{H}(13) & 2.85 & 65702 & \mathrm{H}(10) & \mathrm{H}(12) & 2.98 & 65702 \\ \mathrm{H}(11) & \mathrm{H}(18) & 2.96 & 65602 & \mathrm{H}(12) & \mathrm{H}(13) & 2.90 & 65602 \\ \mathrm{H}(12) & \mathrm{H}(18) & 3.37 & 65602 & \mathrm{H}(14) & \mathrm{H}(15) & 2.73 & 66602 \\ \mathrm{H}(14) & \mathrm{H}(20) & 3.21 & 66602 & \mathrm{H}(14) & \mathrm{H}(14) & 3.40 & 66602 \\ \mathrm{H}(16) & \mathrm{H}(21) & 2.86 & 45501 & \mathrm{H}(17) & \mathrm{H}(21) & 2.67 & 45501 \\ \mathrm{H}(17) & \mathrm{H}(17) & 3.47 & 56602 & \mathrm{H}(18) & \mathrm{H}(21) & 2.95 & 45501 \\ \mathrm{H}(19) & \mathrm{H}(19) & 3.09 & 75602 & \mathrm{H}(19) & \mathrm{H}(21) & 3.34 & 75602 \\ & & & & & & & \end{array}$


The ADC (atom designator code) specifies the position of an atom in a crystal. The 5-digit number shown in the table is a composite of three one-digit numbers and one two-digit number: TA (first digit) + TB (second digit) + TC (third digit) + SN (last two digits). TA, TB and TC are the crystal lattice translation digits along cell edges $a, b$ and c. A translation digit of 5 indicates the origin unit cell. If TA $=4$, this indicates a translation of one unit cell length along the a-axis in the negative direction. Each translation digit can range in value from 1 to 9 and thus \pm 4 lattice translations from the origin ( $\mathrm{TA}=5, \mathrm{~TB}=5, \mathrm{TC}=5)$ can be represented.

The SN, or symmetry operator number, refers to the number of the symmetry operator used to generate the coordinates of the target atom. A list of symmetry operators relevant to this structure is given below.

For a given intermolecular contact, the first atom (origin atom) is located in the origin unit cell and its position can be generated using the identity operator ( $\mathrm{SN}=1$ ). Thus, the ADC for an origin atom is always 55501 . The position of the second atom (target atom) can be generated using the ADC and the coordinates of the atom in the parameter table. For example, an ADC of 47502 refers to the target atom moved through symmetry operator two, then translated -1 cell translations along the a axis, +2 cell translations along the $b$ axis, and 0 cell translations al ong the $c$ axis.

An ADC of 1 indicates an intermolecular contact between two fragments (eg. cation and anion) that reside in the same asymmetric unit.

Symmetry Operators:

$\begin{array}{lllllll}(1) & +X, \quad+Y, & +Z & \text { (2) } & -X, & -Y, & -Z\end{array}$ 


\section{Theoretical Studies of the Effect of Lewis Acid Using MO Calculations}

The energies and coefficients calculated at the reaction sites for carbonyl ylide $\mathbf{A}$ and imines $\mathbf{2 c}-$ d using the ab initio RHF/3-21G method are shown in Figure I and Table II, respectively.

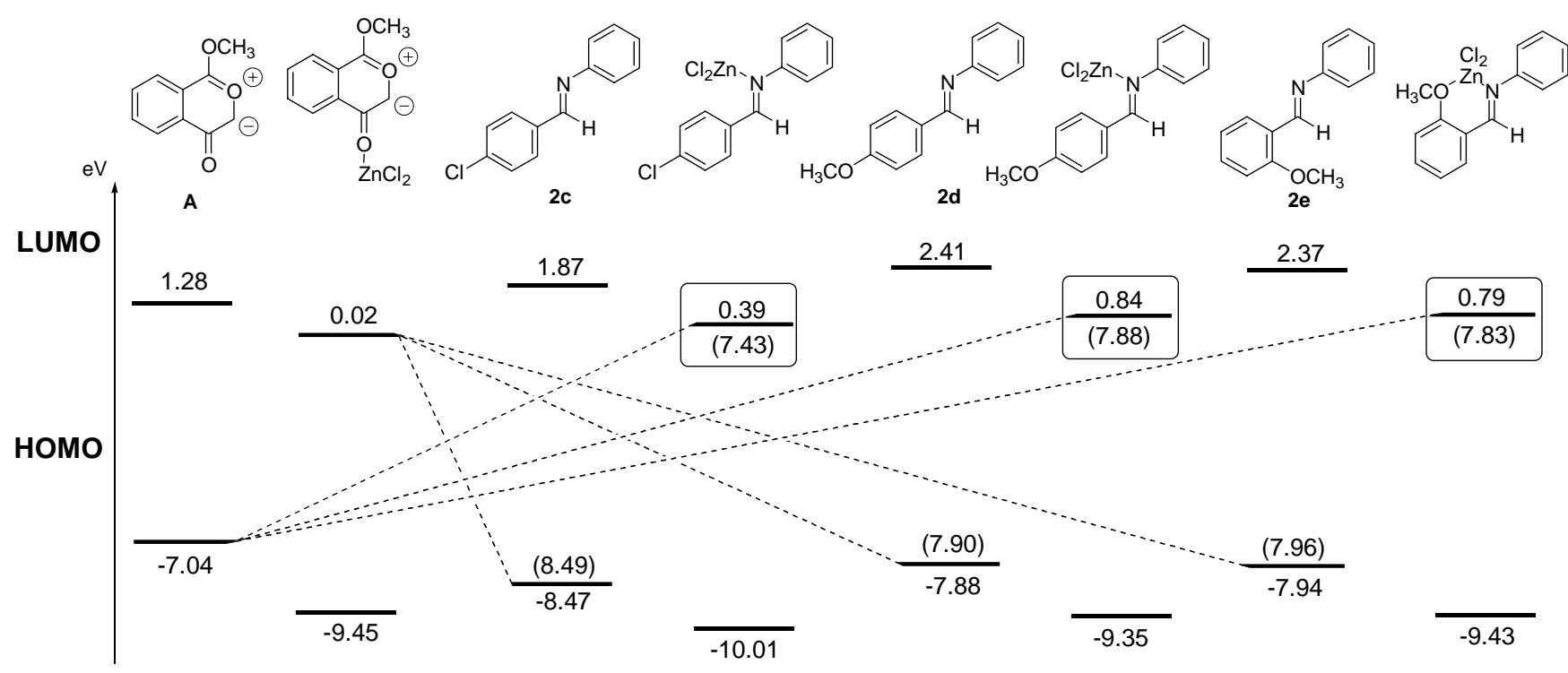

Energy (hartree)

$-604.47$

$-3288.48$

$-1006.76$

$-3690.72$

$-663.30$

$-3347.27$

$-663.30$

$-3347.31$

Figure I. HOMO and LUMO Energies of Carbonyl Ylide A and Imines $\mathbf{2 c}-\mathbf{e}$

Table II. Coefficients of Carbonyl Ylide A and Imines $\mathbf{2 c}-\mathbf{2 e}$<smiles>C[B-]C(=O)c1ccccc1OC</smiles>

\begin{tabular}{|c|c|c|}
\hline HOMO/LUMO & Coefficients & $\beta$ \\
\hline HOMO (2p) & -0.149 & 0.351 \\
\hline HOMO (3p) & -0.174 & 0.494 \\
\hline LUMO (2p) & 0.275 & 0.139 \\
\hline LUMO (3p) & 0.355 & 0.206 \\
\hline $\operatorname{HOMO}(2 \mathrm{p})-\mathrm{ZnCl}_{2}$ & -0.115 & 0.322 \\
\hline
\end{tabular}




\begin{tabular}{lcc}
$\mathrm{HOMO}(3 \mathrm{p})-\mathrm{ZnCl}_{2}$ & -0.129 & 0.404 \\
$\mathrm{LUMO}(2 \mathrm{p})-\mathrm{ZnCl}_{2}$ & 0.292 & 0.123 \\
$\mathrm{LUMO}(3 \mathrm{p})-\mathrm{ZnCl} 2$ & 0.374 & 0.177 \\
\hline
\end{tabular}

${ }_{\mathrm{Ar}^{\prime}}^{\delta}{ }^{\mathrm{N}} \mathrm{Ph}$

\begin{tabular}{|c|c|c|c|}
\hline Imine & HOMO/LUMO ${ }^{\mathrm{a}}$ & Coefficients $\gamma$ & $\delta$ \\
\hline \multirow[t]{8}{*}{$2 c$} & HOMO (2p) & 0.131 & 0.165 \\
\hline & HOMO (3p) & 0.186 & 0.218 \\
\hline & LUMO (2p) & 0.196 & -0.185 \\
\hline & LUMO (3p) & 0.325 & -0.318 \\
\hline & $\operatorname{HOMO}(2 \mathrm{p})-\mathrm{ZnCl}_{2}$ & 0.0711 & 0.129 \\
\hline & $\operatorname{HOMO}(3 p)-\mathrm{ZnCl}_{2}$ & 0.0989 & 0.177 \\
\hline & LUMO (2p)- $\mathrm{ZnCl}_{2}$ & 0.256 & -0.209 \\
\hline & LUMO (3p)- $\mathrm{ZnCl}_{2}$ & 0.429 & -0.365 \\
\hline \multirow[t]{8}{*}{$2 d$} & $\operatorname{HOMO}(2 \mathrm{p})$ & 0.106 & 0.174 \\
\hline & HOMO (3p) & 0.156 & 0.232 \\
\hline & LUMO (2p) & 0.214 & -0.180 \\
\hline & LUMO (3p) & 0.361 & -0.313 \\
\hline & $\operatorname{HOMO}(2 \mathrm{p})-\mathrm{ZnCl}_{2}$ & 0.0320 & 0.159 \\
\hline & $\operatorname{HOMO}(3 p)-\mathrm{ZnCl}_{2}$ & 0.0539 & 0.221 \\
\hline & LUMO (2p)- $\mathrm{ZnCl}_{2}$ & 0.270 & -0.203 \\
\hline & LUMO (3p)- $\mathrm{ZnCl}_{2}$ & 0.454 & -0.358 \\
\hline
\end{tabular}




$\begin{array}{lcc}\operatorname{HOMO}(2 \mathrm{p}) & 0.127 & 0.170 \\ \operatorname{HOMO}(3 \mathrm{p}) & 0.185 & 0.224 \\ \operatorname{LUMO}(2 \mathrm{p}) & 0.203 & -0.181 \\ \mathrm{LUMO}(3 \mathrm{p}) & 0.341 & -0.311 \\ \mathrm{HOMO}(2 \mathrm{p})-\mathrm{ZnCl}_{2} & 0.0962 & 0.112 \\ \mathrm{HOMO}(3 \mathrm{p})-\mathrm{ZnCl}_{2} & 0.127 & 0.152 \\ \mathrm{LUMO}(2 \mathrm{p})-\mathrm{ZnCl}_{2} & 0.237 & -0.211 \\ \mathrm{LUMO}(3 \mathrm{p})-\mathrm{ZnCl}_{2} & 0.400 & -0.365\end{array}$

${ }^{a}$ The sets of coefficients ( $2 p$ and $3 p$ ) for the valence shell were obtained when the calculations were performed using a split valence basis set (3-21G).

Energetics of the cycloaddition of carbonyl ylide $\mathbf{A}$ with imine $\mathbf{2 c}$ by the calculations using the ONIOM (B3LYP/6-31G(d):PM3) method.

\begin{tabular}{|c|c|c|c|}
\hline \multicolumn{4}{|c|}{ Carbonyl Ylide(A) } \\
\hline \multicolumn{4}{|c|}{ ONIOM(B3LYP/6-31g(d):PM3) } \\
\hline \multicolumn{4}{|c|}{ Energy : $\quad-381.548542756443$ au } \\
\hline \multicolumn{4}{|c|}{ Lowest Frequency : $\quad 72.9946 \mathrm{~cm}^{-1}$} \\
\hline \multicolumn{4}{|c|}{ Molecular mass: $\quad 176.04734 \mathrm{amu}$} \\
\hline \multicolumn{4}{|c|}{ Sum of electronic and thermal Energies : $\quad-381.378348$ au } \\
\hline \multicolumn{4}{|c|}{ Sum of electronic and thermal Enthalpies $(\mathrm{H}): \quad-381.377403 \mathrm{at}$} \\
\hline \multicolumn{4}{|c|}{ Sum of electronic and thermal Free Energies $(G): \quad-381.4252$} \\
\hline \multicolumn{4}{|c|}{ Entropy (S) : $100.667 \mathrm{cal} / \mathrm{molK}$} \\
\hline \multicolumn{4}{|c|}{ (Temperature $\quad 298.150$ Kelvin, Pressure } \\
\hline \multicolumn{4}{|c|}{ Cartesian Coordinates } \\
\hline $\mathrm{C}$ & 1.175853 & 0.000025 & 3.563691 \\
\hline 0 & 0.091405 & 0.000002 & 4.198239 \\
\hline $\mathrm{C}$ & 3.631420 & 0.000009 & 2.091561 \\
\hline $\mathrm{C}$ & 2.442379 & 0.000000 & 4.159813 \\
\hline $\mathrm{H}$ & 2.662467 & -0.000023 & 5.215508 \\
\hline 0 & 3.599356 & -0.000001 & 3.393688 \\
\hline 0 & 4.813337 & 0.000011 & 1.463910 \\
\hline
\end{tabular}




$\begin{array}{rrrr}\mathrm{C} & 5.988269 & 0.000000 & 2.293292 \\ \mathrm{H} & 6.011742 & -0.893872 & 2.923151 \\ \mathrm{H} & 6.824664 & 0.000012 & 1.595775 \\ \mathrm{H} & 6.011739 & 0.893853 & 2.923179 \\ \mathrm{C} & 1.192322 & 0.000013 & 2.068744 \\ \mathrm{C} & 2.405922 & 0.000009 & 1.343894 \\ \mathrm{C} & -0.024283 & -0.000007 & -0.024170 \\ \mathrm{C} & -0.019220 & 0.000005 & 1.355545 \\ \mathrm{C} & 2.386181 & 0.000002 & -0.067496 \\ \mathrm{C} & 1.183500 & -0.000009 & -0.738005 \\ \mathrm{H} & -0.973727 & -0.000012 & -0.570638 \\ \mathrm{H} & -0.962979 & 0.000007 & 1.918448 \\ \mathrm{H} & 3.327904 & 0.000002 & -0.627732 \\ \mathrm{H} & 1.164548 & -0.000015 & -1.833146\end{array}$

$N(2$ - methoxybenzylidene)aniline (2c)

ONIOM(B3LYP/6- 31g(d):PM3)

Energy : $\quad-210.237745474887$ au

Lowest Frequency : $\quad 12.7661 \mathrm{~cm}^{-1}$

Molecular mass: $211.09971 \mathrm{amu}$

Sum of electronic and thermal Energies : - 209.989491 au

Sum of electronic and thermal Enthalpies $(H)$ : $\quad-209.988547 \mathrm{au}$

Sum of electronic and thermal Free Energies $(G)$ : $\quad-210.047448$ au

Entropy (S) : $123.968 \mathrm{cal} / \mathrm{molK}$

(Temperature $\quad 298.150$ Kelvin, Pressure 1.00000 Atm)

$\begin{array}{cccc}\text { Cartesian Coordinates } & & & \\ \text { C } & 3.721163 & -0.183850 & -0.652488 \\ \text { H } & 3.693827 & -0.398153 & -1.734848 \\ \text { N } & 4.798644 & 0.057883 & 0.015042 \\ \text { O } & 1.405673 & 0.301061 & -2.041476 \\ \text { C } & 0.230847 & 0.553007 & -2.823745 \\ \text { H } & -0.465650 & -0.298638 & -2.832850 \\ \text { H } & -0.307116 & 1.457371 & -2.503166 \\ \text { H } & 0.602216 & 0.710489 & -3.839049 \\ \text { C } & 2.421546 & -0.179923 & 0.039342 \\ \text { C } & 6.051202 & 0.031709 & -0.748794\end{array}$




$\begin{array}{lrrr}\text { C } & 1.228212 & 0.062625 & -0.683149 \\ \text { C } & -0.010422 & 0.056326 & -0.037244 \\ \text { C } & -0.068327 & -0.191860 & 1.330113 \\ \text { C } & 1.093962 & -0.430350 & 2.050895 \\ \text { C } & 2.329261 & -0.422826 & 1.410257 \\ \text { H } & -0.939985 & 0.244584 & -0.586137 \\ \text { H } & 1.041449 & -0.623552 & 3.127214 \\ \text { H } & 3.240267 & -0.607337 & 1.993292 \\ \text { C } & 6.919821 & 1.117735 & -0.607509 \\ \text { C } & 6.408025 & -1.066572 & -1.536212 \\ \text { C } & 8.140553 & 1.103835 & -1.272432 \\ \text { H } & 6.644970 & 1.972975 & 0.020432 \\ \text { C } & 7.635249 & -1.067351 & -2.189364 \\ \text { H } & 5.733832 & -1.924847 & -1.639028 \\ \text { C } & 8.499044 & 0.015243 & -2.061402 \\ \text { H } & 8.824407 & 1.952936 & -1.170913 \\ \text { H } & 7.921719 & -1.925494 & -2.806063 \\ \text { H } & 9.463272 & 0.009391 & -2.579683 \\ \text { H } & -1.038990 & -0.197354 & 1.837068\end{array}$

Complex of Carbonyl Ylide (A) with $N$ (2- methoxybenzylidene)aniline (2c)

ONIOM(B3LYP/6-31g(d):PM3)

Energy : $\quad 591.798751410191$ au

Lowest Frequency : $6.8163 \mathrm{~cm}^{-1}$

Molecular mass: $387.14706 \mathrm{amu}$

Sum of electronic and thermal Energies : $\quad 591.377294$ au

Sum of electronic and thermal Enthalpies (H) : $\quad-591.376350$ au

Sum of electronic and thermal Free Energies $(G)$ : $\quad$ - 591.466059 au

Entropy (S) : $188.809 \mathrm{cal} / \mathrm{molK}$

(Temperature $\quad 298.150$ Kelvin, Pressure 1.00000 Atm)

Cartesian Coordinates

$\begin{array}{lrrr}\text { C } & -0.123482 & 0.210777 & 0.342817 \\ \text { C } & 0.934629 & 0.480704 & 2.987655 \\ \text { C } & 1.667543 & 0.006183 & 1.902761 \\ \text { H } & 2.676461 & -0.383206 & 1.920330\end{array}$




\begin{tabular}{|c|c|c|c|}
\hline C & 1.070402 & - 4.109623 & 0.947150 \\
\hline $\mathrm{H}$ & 0.740424 & - 4.829297 & 1.715965 \\
\hline 0 & 1.366002 & 0.542611 & 4.174837 \\
\hline 0 & 1.095741 & - 0.123541 & 0.641755 \\
\hline $\mathrm{N}$ & 0.351980 & - 3.752882 & -0.064719 \\
\hline 0 & -0.574844 & 0.012534 & - 0.891507 \\
\hline C & 0.293143 & - 0.717053 & - 1.794484 \\
\hline $\mathrm{H}$ & 0.492911 & -1.714858 & - 1.387924 \\
\hline $\mathrm{H}$ & - 0.270361 & -0.773494 & - 2.724939 \\
\hline $\mathrm{H}$ & 1.224483 & - 0.164221 & - 1.944597 \\
\hline 0 & 1.936642 & - 3.416619 & 3.434168 \\
\hline C & 2.136376 & - 2.715911 & 4.684004 \\
\hline $\mathrm{H}$ & 3.050872 & - 3.046894 & 5.199024 \\
\hline $\mathrm{H}$ & 2.138671 & -1.625082 & 4.560370 \\
\hline $\mathrm{H}$ & 1.274346 & - 3.000376 & 5.292130 \\
\hline C & -0.449643 & 0.931079 & 2.654414 \\
\hline C & - 0.972351 & 0.792969 & 1.348364 \\
\hline C & 2.410224 & - 3.532774 & 1.126827 \\
\hline C & - 0.973007 & - 4.377114 & - 0.167933 \\
\hline C & 2.840603 & - 3.148821 & 2.418367 \\
\hline C & - 1.262705 & 1.500781 & 3.649801 \\
\hline C & - 2.287818 & 1.217959 & 1.065326 \\
\hline C & - 3.060585 & 1.772299 & 2.061401 \\
\hline C & - 2.545838 & 1.915780 & 3.358454 \\
\hline $\mathrm{H}$ & -0.855614 & 1.609082 & 4.664575 \\
\hline $\mathrm{H}$ & - 2.692714 & 1.106383 & 0.053077 \\
\hline $\mathrm{H}$ & - 4.081593 & 2.104199 & 1.844696 \\
\hline $\mathrm{H}$ & - 3.170990 & 2.360208 & 4.140522 \\
\hline C & 3.275120 & - 3.351002 & 0.048628 \\
\hline C & 4.536111 & - 2.795861 & 0.240288 \\
\hline $\mathrm{H}$ & 2.956543 & - 3.643852 & - 0.958552 \\
\hline C & 4.100980 & - 2.572586 & 2.604508 \\
\hline C & 4.941827 & -2.403218 & 1.510382 \\
\hline $\mathrm{H}$ & 5.208156 & - 2.664311 & - 0.613674 \\
\hline $\mathrm{H}$ & 4.430129 & - 2.250223 & 3.599700 \\
\hline C & - 1.885487 & - 4.337373 & 0.889933 \\
\hline C & - 1.325168 & - 4.954795 & - 1.390757 \\
\hline C & - 3.150824 & - 4.887339 & 0.718679 \\
\hline $\mathrm{H}$ & -1.613612 & - 3.877614 & 1.847808 \\
\hline C & - 2.592452 & - 5.505634 & -1.543433 \\
\hline
\end{tabular}




$\begin{array}{lrrr}\mathrm{H} & -0.612016 & -4.978407 & -2.222459 \\ \mathrm{C} & -3.504114 & -5.472260 & -0.492926 \\ \mathrm{H} & -3.871008 & -4.859159 & 1.543100 \\ \mathrm{H} & -2.873966 & -5.966070 & -2.496197 \\ \mathrm{H} & -4.501271 & -5.905922 & -0.620324 \\ \mathrm{H} & 5.931550 & -1.956934 & 1.654724\end{array}$

Transition State for the reaction of Carbonyl Ylide (A) with $N$ (2- methoxybenzylidene)aniline (2c) (exo)

ONIOM(B3LYP/6- 31g(d):PM3)

Energy : $\quad-591.783130525427$ au

Lowest Frequency : $\quad 273.3712 \mathrm{i} \mathrm{cm}^{-1}$

Molecular mass: $387.14706 \mathrm{amu}$

Sum of electronic and thermal Energies : $\quad-591.361899$ au

Sum of electronic and thermal Enthalpies $(H)$ : $\quad-591.361899 \mathrm{au}$

Sum of electronic and thermal Free Energies (G) : $\quad$ - 591.441477 au

Entropy (S) : $169.474 \mathrm{cal} / \mathrm{molK}$

(Temperature $\quad 298.150$ Kelvin, Pressure 1.00000 Atm)

$\begin{array}{crrr}\text { Cartesian Coordinates } & & & \\ \text { C } & 1.041198 & 0.349096 & 3.625872 \\ \text { C } & -1.443621 & -0.637495 & 2.629763 \\ \text { C } & -1.270395 & 0.024317 & 3.884404 \\ \text { H } & -2.110238 & 0.423430 & 4.436022 \\ \text { C } & -0.450921 & -1.713323 & 5.243909 \\ \text { H } & -1.066055 & -2.506312 & 4.781080 \\ \text { O } & -2.520992 & -1.123606 & 2.240009 \\ \text { O } & -0.110012 & 0.747511 & 4.125089 \\ \text { N } & 0.872428 & -1.633023 & 5.048346 \\ \text { O } & 2.150795 & 0.964469 & 4.030589 \\ \text { C } & 2.156880 & 1.420804 & 5.397417 \\ \text { H } & 1.847062 & 0.589444 & 6.045937 \\ \text { H } & 3.187875 & 1.714920 & 5.593242 \\ \text { H } & 1.488837 & 2.278096 & 5.517828 \\ \text { O } & -3.182914 & -1.840757 & 5.736301 \\ \text { C } & -4.474971 & -2.323660 & 6.137699 \\ \text { H } & -4.403432 & -3.257120 & 6.714754 \\ \text { H } & -5.038715 & -1.583262 & 6.731268\end{array}$




\begin{tabular}{|c|c|c|c|}
\hline $\mathrm{H}$ & - 5.008666 & -2.517236 & 5.205048 \\
\hline C & - 0.194893 & -0.754148 & 1.815616 \\
\hline C & 1.041356 & -0.303646 & 2.329230 \\
\hline C & - 1.038344 & - 1.084046 & 6.439831 \\
\hline C & 1.478614 & - 2.691455 & 4.242270 \\
\hline C & -2.420071 & - 1.223557 & 6.720862 \\
\hline C & - 0.235360 & - 1.314434 & 0.531788 \\
\hline C & 2.214480 & -0.462635 & 1.569587 \\
\hline C & 2.149861 & - 1.025963 & 0.309686 \\
\hline C & 0.922881 & - 1.446483 & -0.213329 \\
\hline $\mathrm{H}$ & - 1.198585 & - 1.655768 & 0.129744 \\
\hline $\mathrm{H}$ & 3.180115 & -0.140956 & 1.976615 \\
\hline $\mathrm{H}$ & 3.062804 & - 1.145647 & - 0.283513 \\
\hline $\mathrm{H}$ & 0.883190 & - 1.888784 & - 1.214654 \\
\hline C & -0.254386 & -0.390021 & 7.365958 \\
\hline C & - 0.804981 & 0.126243 & 8.533542 \\
\hline $\mathrm{H}$ & 0.820079 & -0.244647 & 7.167045 \\
\hline C & - 2.972080 & - 0.701848 & 7.894534 \\
\hline C & - 2.159350 & - 0.033007 & 8.800267 \\
\hline $\mathrm{H}$ & -0.166643 & 0.658213 & 9.246144 \\
\hline $\mathrm{H}$ & - 4.049786 & - 0.816771 & 8.084164 \\
\hline C & 0.750009 & - 3.681720 & 3.572975 \\
\hline C & 2.879295 & - 2.719308 & 4.216532 \\
\hline C & 1.418768 & - 4.682396 & 2.879566 \\
\hline $\mathrm{H}$ & - 0.351061 & - 3.666660 & 3.600234 \\
\hline C & 3.535013 & - 3.728534 & 3.522507 \\
\hline $\mathrm{H}$ & 3.457161 & - 1.952062 & 4.744792 \\
\hline C & 2.809015 & - 4.708811 & 2.853097 \\
\hline $\mathrm{H}$ & 0.845968 & -5.453781 & 2.354451 \\
\hline $\mathrm{H}$ & 4.629446 & - 3.753602 & 3.505076 \\
\hline $\mathrm{H}$ & 3.331527 & - 5.501538 & 2.308501 \\
\hline $\mathrm{H}$ & - 2.590806 & 0.372033 & 9.721379 \\
\hline
\end{tabular}

Adduct (exo-3c)

ONIOM(B3LYP/6- 31g(d):PM3)

Energy : $\quad 591.861257656637$ au

Lowest Frequency : $\quad 26.1278 \mathrm{~cm}^{-1}$ 
Molecular mass: $\quad 387.14706 \mathrm{amu}$

Sum of electronic and thermal Energies : $\quad-591.436388$ au

Sum of electronic and thermal Enthalpies $(H)$ : $\quad-591.435444$ au

Sum of electronic and thermal Free Energies (G) : $\quad$ - 591.514605 au

Entropy (S) : $166.610 \mathrm{cal} / \mathrm{molK}$

(Temperature $\quad 298.150$ Kelvin, Pressure 1.00000 Atm)

Cartesian Coordinates

C

$\begin{array}{lll}1.057513 & 0.203997 & 3.395573\end{array}$

C

$-1.378304 \quad-0.345508 \quad 2.071118$

$\mathrm{H}$

$\begin{array}{lll}-1.184420 & 0.056369 \quad 3.534878\end{array}$

C

$\begin{array}{lll}-2.102267 & 0.506780 & 3.914974\end{array}$

$\mathrm{H}$

$\begin{array}{lll}-0.672214 & -1.145578 \quad 4.393189\end{array}$

$-1.041811 \quad-2.105550 \quad 3.981401$

0

$\begin{array}{lll}-2.467492 & -0.612984 & 1.591470\end{array}$

0

$\begin{array}{lll}-0.115896 & 0.996457 & 3.611519\end{array}$

$\mathrm{N}$

$\begin{array}{lll}0.787359 & -0.993056 & 4.232066\end{array}$

0

$2.188333 \quad 0.928845 \quad 3.750830$

C

$\begin{array}{lll}2.183917 & 1.435391 & 5.087642\end{array}$

$\mathrm{H}$

$\begin{array}{lll}1.866626 & 0.659638 & 5.800958\end{array}$

$\mathrm{H}$

$3.215211 \quad 1.731406$

5.292937

$\mathrm{H}$

$1.524771 \quad 2.305338$

5.174235

0

$-3.245848-1.782912$

4.996471

C

$-4.638598 \quad-2.055717$

5.202668

$\mathrm{H}$

$-4.807361-2.920210$

5.861595

$\mathrm{H}$

$-5.187669-1.187779$

5.595975

$\mathrm{H}$

$-5.023139-2.290383$

4.207879

C

$-0.110608-0.392264$

1.275862

C

$1.121506-0.138688$

1.917264

C

$-1.160056-1.030129$

5.805355

$1.597538-2.192910$

4.025821

C

$-2.499393-1.382272$

6.100812

C

- $0.349249-0.594454$

6.849372

$\mathrm{H}$

$\begin{array}{lll}0.690888 & -0.288680 & 6.647724\end{array}$

C

$\begin{array}{lll}-0.129178 & -0.688952 & -0.084068\end{array}$

C

$\begin{array}{lll}2.305546 & -0.203821 & 1.194027\end{array}$

C

$2.272676-0.505923 \quad-0.166992$

C

$1.062645-0.743455-0.803451$

$\mathrm{H}$

$-1.084218-0.881888-0.588403$

$\mathrm{H}$

$3.268229-0.018600 \quad 1.685344$ 


$\begin{array}{lrrr}\text { H } & 3.209006 & -0.553301 & -0.733371 \\ \mathrm{H} & 1.040956 & -0.976548 & -1.873345 \\ \mathrm{C} & -2.987884 & -1.316661 & 7.406704 \\ \mathrm{C} & -0.836583 & -0.526972 & 8.152296 \\ \mathrm{C} & -2.146538 & -0.890471 & 8.429534 \\ \mathrm{H} & -4.021659 & -1.594343 & 7.641248 \\ \mathrm{H} & -0.180320 & -0.184972 & 8.958976 \\ \mathrm{C} & 1.024396 & -3.418802 & 3.669007 \\ \mathrm{C} & 2.975197 & -2.115157 & 4.265581 \\ \mathrm{C} & 1.823182 & -4.548499 & 3.545342 \\ \mathrm{H} & -0.059977 & -3.494181 & 3.495171 \\ \mathrm{C} & 3.762999 & -3.252326 & 4.134202 \\ \mathrm{H} & 3.439833 & -1.163499 & 4.553035 \\ \mathrm{C} & 3.192264 & -4.468525 & 3.774688 \\ \mathrm{H} & 1.369401 & -5.504920 & 3.265482 \\ \mathrm{H} & 4.840343 & -3.188459 & 4.319606 \\ \mathrm{H} & 3.818448 & -5.360768 & 3.675079 \\ \mathrm{H} & -2.525559 & -0.840278 & 9.455576\end{array}$

Zincchloride $\left(\mathrm{ZnCl}_{2}\right)$

B3LYP/6- 31g(d)

Energy : - 2699.61358681 au

Lowest Frequency : $\quad 90.4834 \mathrm{~cm}^{-1}$

Molecular mass: $133.86685 \mathrm{amu}$

Sum of electronic and thermal Energies : - 2699.606852 au

Sum of electronic and thermal Enthalpies $(H)$ : $\quad-2699.605908$ au

Sum of electronic and thermal Free Energies (G) : $\quad$ - 2699.638220 au

Entropy (S) : $68.006 \mathrm{cal} / \mathrm{molK}$

(Temperature $\quad 298.150$ Kelvin, Pressure 1.00000 Atm)

Cartesian Coordinates

$\begin{array}{lccc}\text { Zn } & 0.570819 & 0.000000 & 0.266585 \\ \mathrm{Cl} & -0.323133 & 0.000000 & 2.146564 \\ \mathrm{Cl} & 1.464732 & 0.000000 & -1.613444\end{array}$


$N$ (2- methoxybenzylidene)aniline- $\mathrm{ZnCl}_{2}$ complex $\left(2 \mathrm{c}-\mathrm{ZnCl}_{2}\right)$

ONIOM(B3LYP/6- 31g(d):PM3)

Energy : $\quad-2909.886404024279$ au

Lowest Frequency : $\quad 24.7171 \mathrm{~cm}^{-1}$

Molecular mass: $344.96656 \mathrm{amu}$

Sum of electronic and thermal Energies : $\quad$ - 2909.628411 au

Sum of electronic and thermal Enthalpies $(H)$ : $\quad-2909.627466$ au

Sum of electronic and thermal Free Energies (G) : - 2909.698343 au

Entropy (S) : $149.172 \mathrm{cal} / \mathrm{molK}$

(Temperature $\quad 298.150$ Kelvin, Pressure 1.00000 Atm)

Cartesian Coordinates

$\begin{array}{lrrr}\mathrm{C} & 3.711889 & 0.037179 & -0.770679 \\ \mathrm{H} & 4.523854 & 0.532658 & -0.198689 \\ \mathrm{~N} & 3.949035 & -0.431715 & -1.951733 \\ \mathrm{Zn} & 2.739596 & -1.438965 & -3.325862 \\ \mathrm{Cl} & 2.695730 & -3.588174 & -2.870017 \\ \mathrm{Cl} & 2.358916 & -0.408955 & -5.244259 \\ \mathrm{O} & 1.135658 & -0.350858 & -2.122934 \\ \mathrm{C} & -0.119192 & 0.075396 & -2.733695 \\ \mathrm{H} & -0.966016 & -0.497167 & -2.329941 \\ \mathrm{H} & -0.277311 & 1.147614 & -2.576876 \\ \mathrm{H} & 0.017097 & -0.125924 & -3.795219 \\ \mathrm{C} & 2.416618 & -0.088988 & -0.100179 \\ \mathrm{C} & 5.318050 & -0.306071 & -2.485044 \\ \mathrm{C} & 1.167460 & -0.290642 & -0.727206 \\ \mathrm{H} & 3.394396 & 0.148732 & 1.818608 \\ \mathrm{C} & -0.003251 & -0.423685 & 0.034222 \\ \mathrm{C} & 0.058820 & -0.359205 & 1.417020 \\ \mathrm{C} & 1.281344 & -0.148010 & 2.051722 \\ \mathrm{C} & 2.439428 & -0.013430 & 1.302876 \\ \mathrm{H} & -0.964419 & -0.589212 & -0.478105 \\ \mathrm{H} & 1.327203 & -0.092273 & 3.144662 \\ \mathrm{C} & 6.319776 & 0.446887 & -1.859641 \\ \mathrm{C} & 5.586817 & -0.994237 & -3.677079 \\ \mathrm{C} & 7.585367 & 0.512776 & -2.425697 \\ \mathrm{H} & 6.107148 & 0.981215 & -0.920694 \\ \mathrm{C} & 6.864446 & -0.918274 & -4.228871 \\ \mathrm{H} & 4.823229 & -1.605355 & -4.209771 \\ & & & \\ & & & \end{array}$




$\begin{array}{rrrr}\mathrm{C} & 7.858563 & -0.168837 & -3.608580 \\ \mathrm{H} & 8.369147 & 1.103011 & -1.938827 \\ \mathrm{H} & 7.085466 & -1.451906 & -5.160761 \\ \mathrm{H} & 8.858930 & -0.113785 & -4.051791 \\ \mathrm{H} & -0.855180 & -0.474935 & 2.009901\end{array}$

Complex of Carbonyl Ylide (A) with $\mathrm{N}$ (2- methoxybenzylidene)aniline- $\mathrm{ZnCl}_{2}$ complex (2c- $\mathrm{ZnCl}_{2}$ )

ONIOM(B3LYP/6-31g(d):PM3)

Energy : $\quad 3291.454972391462$ au

Lowest Frequency : $\quad 12.0670 \mathrm{~cm}^{-1}$

Molecular mass: $521.01391 \mathrm{amu}$

Sum of electronic and thermal Energies : $\quad 3291.023626$ au

Sum of electronic and thermal Enthalpies $(H)$ : $-3291.022682 \mathrm{au}$

Sum of electronic and thermal Free Energies $(G)$ : $\quad$ - 3291.122741 au

Entropy (S) : $210.593 \mathrm{cal} / \mathrm{molK}$

(Temperature $\quad 298.150$ Kelvin, Pressure 1.00000 Atm)

Cartesian Coordinates

$\begin{array}{lccc}\mathrm{C} & 1.234445 & 1.977669 & 1.175765 \\ \mathrm{C} & -0.828074 & 1.007805 & -1.227230 \\ \mathrm{H} & 0.059112 & 1.523179 & -1.655034 \\ \mathrm{~N} & -0.883337 & -0.288319 & -1.187518 \\ \mathrm{O} & -3.564916 & 0.144914 & -0.439133 \\ \mathrm{Zn} & -2.123423 & -1.545056 & -0.131164 \\ \mathrm{Cl} & -1.386857 & -1.633340 & 1.975139 \\ \mathrm{Cl} & -3.315778 & -3.143333 & -1.100056 \\ \mathrm{H} & 0.274382 & 2.468178 & 1.250803 \\ \mathrm{C} & 2.120742 & 2.028665 & 0.105072 \\ \mathrm{O} & 1.906143 & 2.655342 & -0.980872 \\ \mathrm{O} & 1.457191 & 1.137197 & 2.264633 \\ \mathrm{C} & 2.508731 & 0.392653 & 2.414250 \\ \mathrm{O} & 2.590257 & -0.423888 & 3.452007 \\ \mathrm{C} & 1.408915 & -0.544288 & 4.290385 \\ \mathrm{H} & 0.556136 & -0.860231 & 3.683702 \\ \mathrm{H} & 1.675394 & -1.301177 & 5.025463 \\ \mathrm{H} & 1.202791 & 0.412688 & 4.775795 \\ \mathrm{C} & -4.983828 & -0.067672 & -0.710391 \\ \mathrm{H} & -5.604236 & 0.391295 & 0.072329\end{array}$




\begin{tabular}{|c|c|c|c|}
\hline $\mathrm{H}$ & -5.250723 & 0.338123 & - 1.691703 \\
\hline $\mathrm{H}$ & - 5.098035 & - 1.150547 & - 0.713805 \\
\hline C & - 1.880618 & 1.868496 & - 0.680123 \\
\hline C & 0.239398 & - 1.041364 & - 1.778494 \\
\hline C & - 3.185277 & 1.485332 & -0.298682 \\
\hline C & 3.388182 & 1.270147 & 0.307852 \\
\hline C & 3.576052 & 0.455060 & 1.447520 \\
\hline C & - 1.529585 & 3.223171 & - 0.530663 \\
\hline C & - 2.422941 & 4.149161 & - 0.015380 \\
\hline $\mathrm{H}$ & - 0.519699 & 3.552558 & - 0.825402 \\
\hline C & - 4.085570 & 2.426727 & 0.222437 \\
\hline C & - 3.701282 & 3.750354 & 0.366986 \\
\hline $\mathrm{H}$ & - 2.120560 & 5.196621 & 0.091260 \\
\hline $\mathrm{H}$ & - 5.095995 & 2.102861 & 0.518364 \\
\hline C & 1.266331 & -0.436055 & -2.513080 \\
\hline C & 0.228180 & - 2.430702 & - 1.584632 \\
\hline C & 2.273988 & - 1.221158 & - 3.058184 \\
\hline $\mathrm{H}$ & 1.289727 & 0.660232 & - 2.641556 \\
\hline C & 1.250448 & - 3.199851 & - 2.135700 \\
\hline $\mathrm{H}$ & -0.572758 & -2.958116 & - 1.018011 \\
\hline C & 2.267025 & -2.600120 & - 2.872195 \\
\hline $\mathrm{H}$ & 3.076411 & -0.747750 & - 3.634256 \\
\hline $\mathrm{H}$ & 1.249643 & - 4.286475 & - 1.991867 \\
\hline $\mathrm{H}$ & 3.062771 & - 3.214635 & - 3.306714 \\
\hline $\mathrm{H}$ & -4.405285 & 4.480458 & 0.780837 \\
\hline C & 4.779108 & -0.262369 & 1.611901 \\
\hline $\mathrm{H}$ & 4.920993 & - 0.899797 & 2.492389 \\
\hline C & 4.422626 & 1.355970 & - 0.639173 \\
\hline $\mathrm{H}$ & 4.277549 & 1.993091 & - 1.522395 \\
\hline C & 5.775682 & -0.159564 & 0.665782 \\
\hline $\mathrm{H}$ & 6.712197 & -0.713739 & 0.791784 \\
\hline C & 5.598296 & 0.654656 & - 0.461999 \\
\hline $\mathrm{H}$ & 6.400801 & 0.730855 & - 1.203991 \\
\hline
\end{tabular}

Transition State for the reaction of Carbonyl Ylide (A) with $\mathrm{N}$ (2- methoxybenzylidene)aniline- $\mathrm{ZnCl}_{2}$ complex $\left(2 \mathrm{c}-\mathrm{ZnCl}_{2}\right)(e x o)$ 


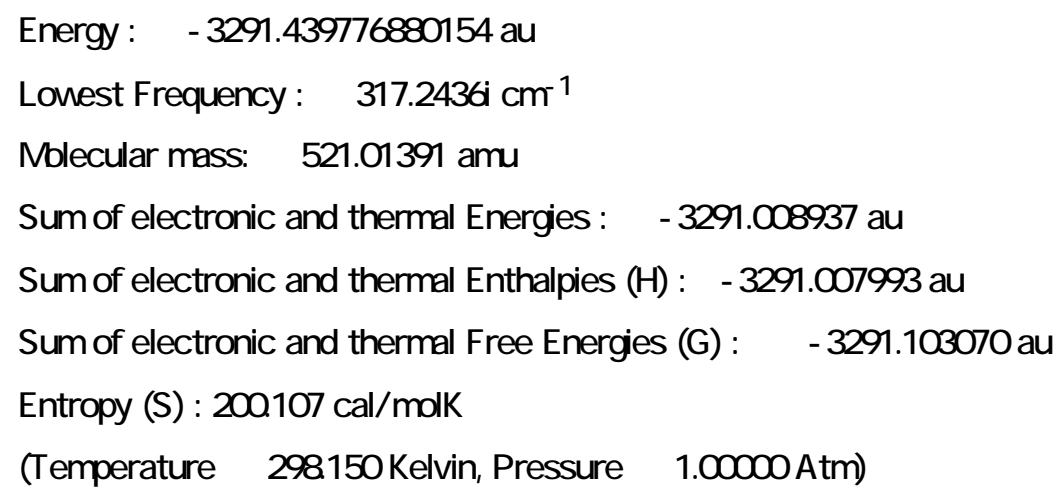




$\begin{array}{lrrr}\text { C } & -3.715353 & 2.337798 & 0.487676 \\ \mathrm{C} & -3.795193 & 3.581425 & -0.118135 \\ \mathrm{H} & -2.820785 & 5.000729 & -1.422666 \\ \mathrm{H} & -4.530407 & 2.000685 & 1.146648 \\ \mathrm{C} & 1.603301 & -0.159826 & -2.559840 \\ \mathrm{C} & 0.781684 & -2.221569 & -1.573668 \\ \mathrm{C} & 2.489392 & -0.892152 & -3.340511 \\ \mathrm{H} & 1.572140 & 0.937262 & -2.654468 \\ \mathrm{C} & 1.676365 & -2.937454 & -2.363292 \\ \mathrm{H} & 0.125363 & -2.800079 & -0.881834 \\ \mathrm{C} & 2.530469 & -2.277933 & -3.242745 \\ \mathrm{H} & 3.152105 & -0.371013 & -4.039445 \\ \mathrm{H} & 1.704203 & -4.031062 & -2.297365 \\ \mathrm{H} & 3.227216 & -2.851458 & -3.862621 \\ \mathrm{H} & -4.672129 & 4.215465 & 0.050885 \\ \mathrm{C} & 3.765714 & -0.842531 & 1.367395 \\ \mathrm{H} & 3.510270 & -1.660851 & 2.052453 \\ \mathrm{C} & 4.430056 & 1.219722 & -0.399389 \\ \mathrm{H} & 4.689637 & 2.025803 & -1.099267 \\ \mathrm{C} & 4.990230 & -0.847165 & 0.716438 \\ \mathrm{H} & 5.695476 & -1.668128 & 0.889650 \\ \mathrm{C} & 5.326784 & 0.186462 & -0.156106 \\ \mathrm{H} & 6.300014 & 0.179556 & -0.660389\end{array}$

Adduct (exo $3 \mathrm{c}$ ) - $\mathrm{ZnCl}_{2}$ complex

ONIOM(B3LYP/6- 31g(d):PM3)

Energy : $\quad 3291.489081645545$ au

Lowest Frequency : $\quad 17.8844 \mathrm{~cm}^{-1}$

Molecular mass: $521.01391 \mathrm{amu}$

Sum of electronic and thermal Energies : - 3291.055398 au

Sum of electronic and thermal Enthalpies $(H)$ : -3291.054454 au

Sum of electronic and thermal Free Energies $(G)$ : $\quad$ - 3291.146295 au

Entropy (S) : $193.296 \mathrm{cal} / \mathrm{molK}$

(Temperature $\quad 298.150$ Kelvin, Pressure 1.00000 Atm)

Cartesian Coordinates

$\begin{array}{llll}\text { C } & 0.654251 & 2.120341 & 1.197444\end{array}$ 


\begin{tabular}{|c|c|c|c|}
\hline C & 0.220770 & 1.529214 & - 0.199628 \\
\hline $\mathrm{H}$ & 0.954109 & 1.850860 & - 0.974441 \\
\hline $\mathrm{N}$ & 0.365569 & 0.049378 & 0.036777 \\
\hline 0 & - 2.204155 & 0.893934 & 1.216975 \\
\hline $\mathrm{Zn}$ & -1.490270 & - 1.086076 & 0.573378 \\
\hline $\mathrm{Cl}$ & -1.881751 & -2.242953 & 2.425475 \\
\hline $\mathrm{Cl}$ & - 2.585134 & - 1.432200 & - 1.342092 \\
\hline $\mathrm{H}$ & - 0.053019 & 2.838452 & 1.612815 \\
\hline C & 2.048123 & 2.730256 & 1.094402 \\
\hline 0 & 2.283588 & 3.922918 & 1.161729 \\
\hline 0 & 0.748402 & 0.993661 & 2.067161 \\
\hline C & 1.282469 & - 0.030306 & 1.242855 \\
\hline 0 & 1.114370 & - 1.268438 & 1.798456 \\
\hline C & 1.601902 & - 1.465354 & 3.135825 \\
\hline $\mathrm{H}$ & 1.130085 & -0.758760 & 3.821493 \\
\hline $\mathrm{H}$ & 1.309027 & - 2.480964 & 3.396676 \\
\hline $\mathrm{H}$ & 2.694980 & - 1.362119 & 3.170348 \\
\hline C & - 3.232480 & 1.062761 & 2.236666 \\
\hline $\mathrm{H}$ & - 3.058165 & 1.987879 & 2.794865 \\
\hline $\mathrm{H}$ & - 4.235180 & 1.071724 & 1.787147 \\
\hline $\mathrm{H}$ & - 3.117784 & 0.192928 & 2.882422 \\
\hline C & - 1.133277 & 1.984721 & - 0.618180 \\
\hline C & 0.863791 & - 0.739167 & - 1.131444 \\
\hline C & - 2.326325 & 1.713545 & 0.082640 \\
\hline C & 3.101481 & 1.690126 & 0.879082 \\
\hline C & 2.733293 & 0.326398 & 0.910754 \\
\hline C & - 1.221542 & 2.787787 & - 1.763004 \\
\hline C & -2.435227 & 3.316718 & - 2.181357 \\
\hline $\mathrm{H}$ & - 0.313764 & 3.005538 & - 2.340401 \\
\hline C & - 3.549992 & 2.254992 & - 0.330270 \\
\hline C & - 3.598691 & 3.052983 & - 1.464553 \\
\hline $\mathrm{H}$ & - 2.476275 & 3.940086 & - 3.080883 \\
\hline $\mathrm{H}$ & - 4.464588 & 2.026188 & 0.239242 \\
\hline C & 1.588349 & - 0.166046 & - 2.182100 \\
\hline C & 0.566015 & - 2.109474 & - 1.145742 \\
\hline C & 2.020674 & - 0.959589 & - 3.237215 \\
\hline $\mathrm{H}$ & 1.812175 & 0.911698 & - 2.185871 \\
\hline C & 1.012818 & - 2.891781 & - 2.209762 \\
\hline $\mathrm{H}$ & 0.001567 & - 2.610808 & -0.328376 \\
\hline C & 1.736486 & -2.321353 & -3.251260 \\
\hline
\end{tabular}




$\begin{array}{lrrr}\mathrm{H} & 2.583022 & -0.507416 & -4.061073 \\ \mathrm{H} & 0.779241 & -3.962609 & -2.229946 \\ \mathrm{H} & 2.076079 & -2.942701 & -4.086918 \\ \mathrm{H} & -4.556235 & 3.468037 & -1.797216 \\ \mathrm{C} & 3.699142 & -0.646977 & 0.684556 \\ \mathrm{H} & 3.426171 & -1.710012 & 0.701970 \\ \mathrm{C} & 4.424690 & 2.046244 & 0.638796 \\ \mathrm{H} & 4.711111 & 3.105619 & 0.624422 \\ \mathrm{C} & 5.021381 & -0.279035 & 0.435151 \\ \mathrm{H} & 5.774041 & -1.055309 & 0.257335 \\ \mathrm{C} & 5.384139 & 1.059840 & 0.417149 \\ \mathrm{H} & 6.424822 & 1.346069 & 0.228858\end{array}$


Proposed mechanism for formation of dimeric product 4

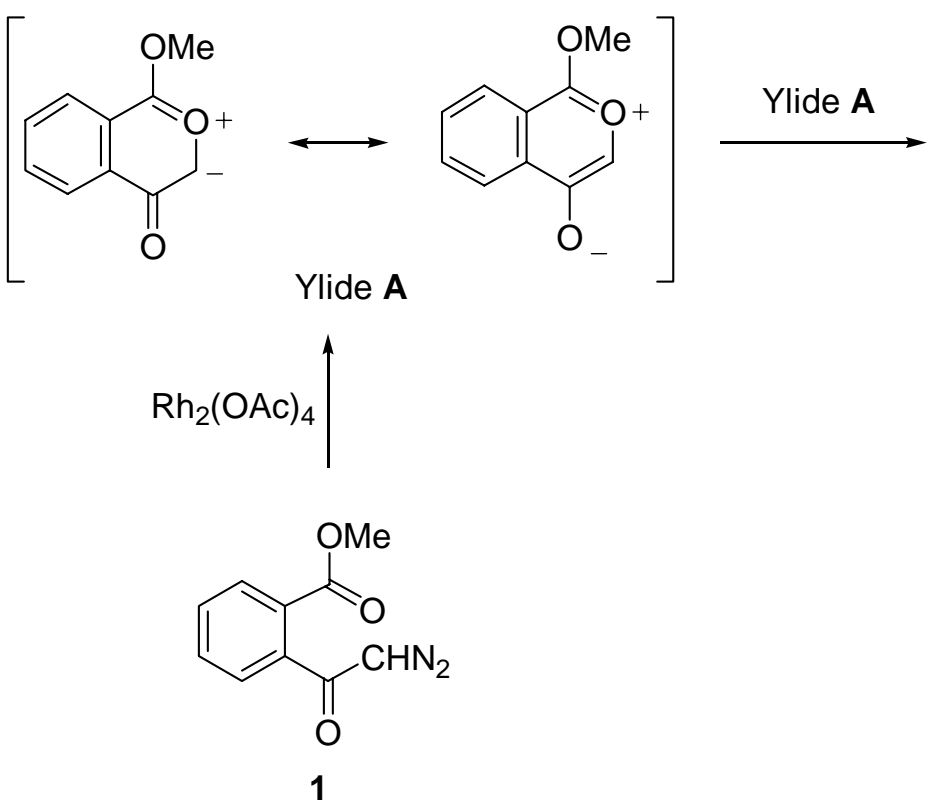

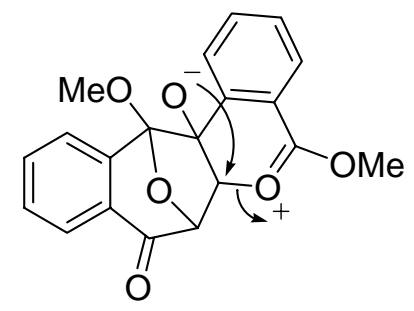<smiles>COC(=O)c1ccccc1C12OC1C1OC2(OC)c2ccccc2C1=O</smiles>

Proposed mechanism for formation of by-product 7

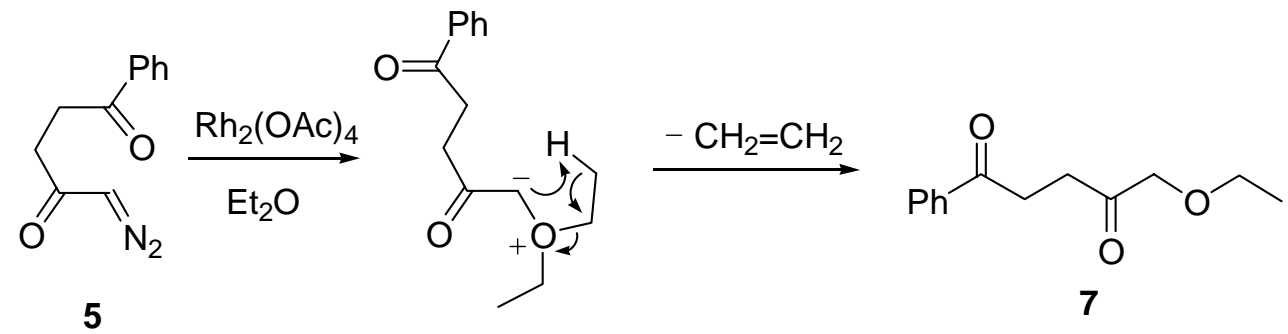

\section{REFERENCES.}

1. (a) Suga, H.; Kakehi, A.; Ito, S.; Inoue, K.; Ishida, H.; Ibata, T. Org. Lett. 2000, 2, $3145 . \quad$ (b) Suga, H.; Kakehi, A.; Ito, S.; Inoue, K.; Ishida, H.; Ibata, T. Bull. Chem. Soc. Jpn. 2001, 74, 1115. (c) Ueda, K.; Ibata, T.; Takebayashi, M. Bull. Chem. Soc. Jpn. 1972, 45, 2779. (d) Ibata, T.; Toyoda, J.; Sawada, M.; Takai, Y.; Tanaka, T. Tetrahedron Lett. 1988, 29, 317.

2. Padwa, A.; Fryxell, G. E.; Zhi, L. J. Am. Chem. Soc. 1990, 112, 3100.

3. Simion, A.; Simion, C.; Kanda, T.; Nagashima, S.; Mitoma, Y.; Yamada, T.; Mimura, K.; Tashiro, M. J. Chem. Soc., Perkitn Trans. 1 2001, 2071.

4. Lautens, M.; Tayama, E.; Nguyen, D. Org. Lett. 2004, 6, 345.

5. Gaussian 03, Revision C.02, Frisch, M. J.; Trucks, G. W.; Schlegel, H. B.; Scuseria, G. E.; Robb, M. A.; Cheeseman, J. R.; Montgomery, Jr., J. A.; Vreven, T.; Kudin, K. N.; Burant, J. C.; 
Millam, J. M.; Iyengar, S. S.; Tomasi, J.; Barone, V.; Mennucci, B.; Cossi, M.; Scalmani, G.; Rega, N.; Petersson, G. A.; Nakatsuji, H.; Hada, M.; Ehara, M.; Toyota, K.; Fukuda, R.; Hasegawa, J.; Ishida, M.; Nakajima, T.; Honda, Y.; Kitao, O.; Nakai, H.; Klene, M.; Li, X.; Knox, J. E.; Hratchian, H. P.; Cross, J. B.; Bakken, V.; Adamo, C.; Jaramillo, J.; Gomperts, R.; Stratmann, R. E.; Yazyev, O.; Austin, A. J.; Cammi, R.; Pomelli, C.; Ochterski, J. W.; Ayala, P. Y.; Morokuma, K.; Voth, G. A.; Salvador, P.; Dannenberg, J. J.; Zakrzewski, V. G.; Dapprich, S.; Daniels, A. D.; Strain, M. C.; Farkas, O.; Malick, D. K.; Rabuck, A. D.; Raghavachari, K.; Foresman, J. B.; Ortiz, J. V.; Cui, Q.; Baboul, A. G.; Clifford, S.; Cioslowski, J.; Stefanov, B. B.; Liu, G.; Liashenko, A.; Piskorz, P.; Komaromi, I.; Martin, R. L.; Fox, D. J.; Keith, T.; Al-Laham, M. A.; Peng, C. Y.; Nanayakkara, A.; Challacombe, M.; Gill, P. M. W.; Johnson, B.; Chen, W.; Wong, M. W.; Gonzalez, C.; and Pople, J. A.; Gaussian, Inc., Wallingford CT, 2004.

6. (a) Svensson, M.; Humbel, S.; Froese, R.D.J.; Matsubara, T.; Sieber, S.; Morokuma, K. J. Phys. Chem. 1996, 100, 19357. (b) Humbel, S.; Sieber, S.; Morokuma, K. J. Chem. Phys. 1996, 105, 1959. 